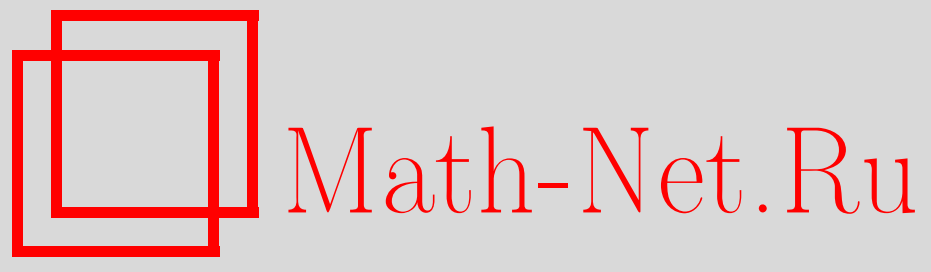

С. В. Болотин, Д. В. Трещёв, Антиинтегрируемый предел, УМН, 2015, том 70, выпуск 6, 3-62

DOI: https://doi.org/10.4213/rm9692

Использование Общероссийского математического портала Math-Net.Ru подразумевает, что вы прочитали и согласны с пользовательским соглашением http://www . mathnet.ru/rus/agreement

Параметры загрузки:

IP : 35.174 .16 .151

26 апреля 2023 г., 18:01:13

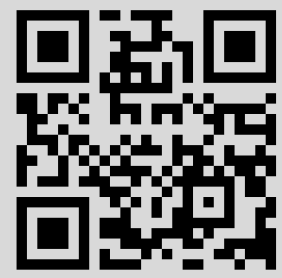


УДК 531.01

\section{Антиинтегрируемый предел}

\section{С. В. Болотин, Д. В. Трещёв}

Антиинтегрируемый предел является одним из удобных и относительно простых методов построения хаотических гиперболических инвариантных множеств в лагранжевых, гамильтоновых и других динамических системах. В настоящей статье рассматривается наиболее естественный контекст метода - дискретные лагранжевы системы. Затем приводятся примеры и приложения.

Библиография: 75 названий.

Ключевые слова: лагранжевы системы, гамильтоновы системы, хаос, гиперболические множества, топологическая марковская цепь, топологическая энтропия.

DOI: $10.4213 / \mathrm{rm} 9692$

\section{СОДЕРЖАНИЕ}

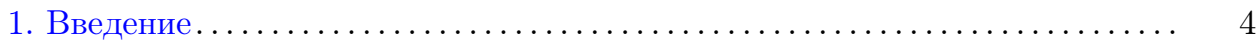

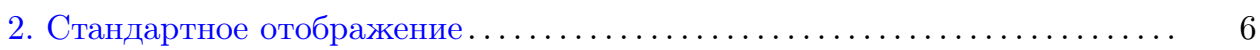

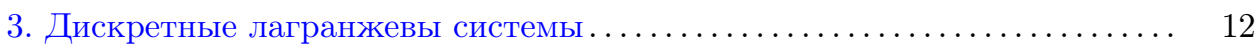

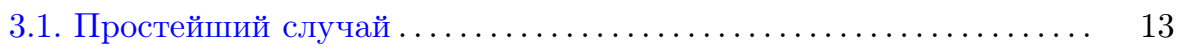

3.2. Многозначные лагранжианы . . . . . . . . . . . . . . . . . 15

3.3. ДЛС, порожденная симплектическим отображением .......... 16

3.4. ДЛС, порожденная лагранжевым потоком ................ 18

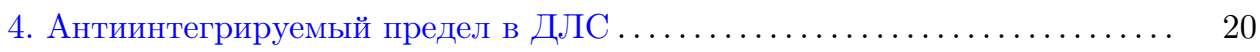

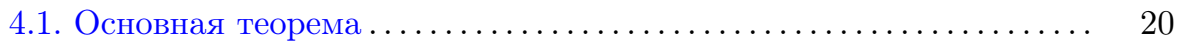

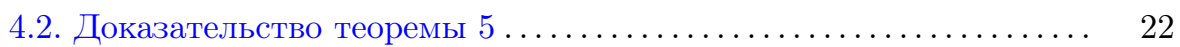

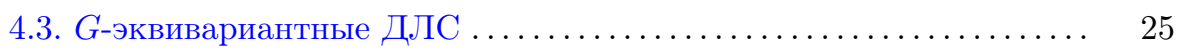

5. Примеры: системы с дискретным временем ................. 26

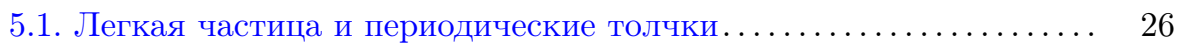

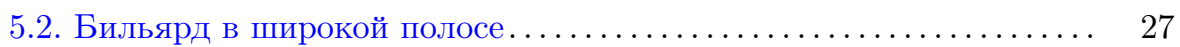

5.3. Бильярды с малыми рассеивателями ................. 29

5.4. Теорема Биркгофа-Смейла-Шильникова.............. 32

5.5. Цепочки гетероклинических траекторий инвариантных торов . . 35

Исследование выполнено за счет гранта Российского научного фонда (проект № 14-50-00005).

(C) С. В. Болотин, Д. В. Трещёв, 2015 
6. Антиинтегрируемый предел в непрерывных лагранжевых системах... 39

6.1. Теорема Тураева-Шильникова...................... 40

6.2. Задача $n$ центров с малыми массами .................. 43

6.3. Лагранжевы системы, медленно зависящие от времени ....... 47

7. Сепаратрисное отображение........................... 52

7.1. Антиинтегрируемый предел в сепаратрисном отображении Заславского ........................................ 52

7.2. Сепаратрисное отображение и диффузия Арнольда.......... 55

Список литературы . . . . . . . . . . . . . . . . . . . . . . . 58

\section{1. Введение}

Развитие математики неравномерно и непредсказуемо. Неожиданные удивительные прорывы чередуются со странными задержками. Такая труднообъяснимая задержка случилась и с появлением общего понятия антиинтегрируемого предела. Теория динамических систем была технически готова к введению такого понятия уже 100 лет назад частично и 50 лет назад полностью. Основные идеи содержатся в работах Пуанкаре, Биркгофа, Хедлунда, Морса и других создателей символической динамики. Замечания о гиперболичности инвариантного множества, появляющегося в антиинтегрируемом пределе, получаются более или менее автоматически. Для этого требуются лишь общее понятие гиперболичности и стандартные технические средства типа конического критерия гиперболичности. Тем не менее антиинтегрируемый предел как универсальный подход появился только в конце прошлого века.

Исходной точкой для формирования идеологии антиинтегрируемого предела послужила статья С. Обри и Ж. Абрамовичи [6], в которой был введен термин "антиинтегрируемый предел" и были зафиксированы основные черты метода: контекст (дискретные лагранжевы системы), основной технический инструмент (принцип сжимающих отображений) и язык представления результатов (символическая динамика).

Напомним, что стандартным отображением называется сохраняющее площадь отображение цилиндра $\{(x, y): x \in \mathbb{T}, y \in \mathbb{R}\}, \mathbb{T}=\mathbb{R} /(2 \pi \mathbb{Z})$, на себя, заданное формулой

$$
\left(\begin{array}{l}
x \\
y
\end{array}\right) \mapsto\left(\begin{array}{l}
x_{+} \\
y_{+}
\end{array}\right)=\left(\begin{array}{c}
x+y+\lambda \sin x \\
y+\lambda \sin x
\end{array}\right)
$$

Если требуется компактность, можно взять в качестве фазового пространства тор $\mathbb{T}^{2}$.

При $\lambda=0$ отображение интегрируемо: переменная $y$ остается постоянной на траекториях. Случай малого $|\lambda|$ широко изучался в физической и математической литературе. Предел $\lambda \rightarrow \infty$ называется антиинтегрируемым пределом [6]. Основным результатом статьи [6] является построение при больших $|\lambda|$ несчетного множества траекторий, находящихся во взаимно однозначном соответствии с некоторым множеством квазитраекторий (кодов). 
Антиинтегрируемый предел появился как реализация той простой идеи, что естественно ожидать усиления хаотичности, когда "расстояние до множества интегрируемых систем", что бы это ни означало, растет.

Отсутствие аналитического первого интеграла в системах, близких к антиинтегрируемому пределу, интуитивно очевидно. Однако формальное доказательство этого факта требует определенной работы ${ }^{1}$. C другой стороны, не следует думать, что неограниченный рост хаоса ведет к эргодичности системы. Действительно, число эллиптических периодических точек может быть сколь угодно большим для больших значений $\lambda$ и, более того, для некоторой возрастающей последовательности значений параметра эти точки оказываются асимптотически плотными на фазовом торе [41]. Поскольку в общей ситуации эллиптические периодические траектории окружены островками устойчивости, стандартное отображение не становится эргодичным при больших $|\lambda|$. Хотя в численных экспериментах при больших значениях параметра $\lambda$ динамика выглядит все более и более хаотичной, строго говоря, не известно даже, является ли мера хаотического множества положительной. Является ли, например, метрическая энтропия стандартного отображения положительной хотя бы для одного значения $\lambda$ ?

Легко показать, что траектории, построенные в антиинтегрируемом пределе, образуют равномерно гиперболическое канторово множество. Хотя это множество несчетно, его мера равна нулю. Стандартным способом количественного исследования хаоса является вычисление (или оценивание) топологической энтропии, которая, в отличие от метрической энтропии, допускает относительно простые положительные оценки снизу. Эти оценки основаны на стандартном факте, состоящем в том, что если система имеет инвариантное множество с динамикой, сопряженной топологической марковской цепи, то топологическая энтропия системы не меньше, чем у этой топологической марковской цепи. В случае стандартного отображения (1.1), рассуждая таким образом, можно получить, что при больших $\lambda$ топологическая энтропия больше $c \log \lambda$ для некоторой положительной постоянной $c$ (ср. с [54]).

План статьи следующий. В разделе 2 мы приводим основные идеи, методы и результаты антиинтегрируемого предела в простейшем нетривиальном примере: в стандартном отображении. Мы определяем соответствующее множество квазитраекторий (кодов) и доказываем, что для каждого кода существует единственная траектория стандартного отображения, отслеживающая эту квазитраекторию. Множество траекторий, полученных таким способом, образует гиперболическое множество. Мы показываем, что топологическая энтропия, которую несет это множество, - порядка $\log \lambda$. В разделе 3 мы определяем класс систем, для которых будут развиваться методы антиинтегрируемого предела. Эти системы называются дискретными лагранжевыми системами (ДЛС). Мы начнем с глобально определенных ДЛС (п. 3.1), а затем объясним, как дискретные лагранжевы системы могут порождаться так называемыми объемлющими

\footnotetext{
${ }^{1}$ В случае двумерного фазового пространства неинтегрируемость следует из существования трансверсальных гомоклиник к периодическим решениям.
} 
системами. В п. 3.3 объемлющими системами являются симплектические отображения.

В разделе 4 представлены теоремы об антиинтегрируемом пределе для ДЛС общего вида. На этих теоремах основаны дальнейшие приложения метода. Затем мы приводим примеры систем, которые можно изучать методами антиинтегрируемого предела. В каждом из примеров имеется большой параметр, аналог $\lambda$ из стандартного отображения. Мы старались расположить примеры в порядке нарастания сложности. Первый набор примеров (раздел 5) содержит системы с дискретным временем. Мы начнем с задачи о движении легкой частицы в поле потенциала с $\delta$-образной зависимостью от $t$. Затем рассмотрим бильярд в полосе со стенками, образованными графиками периодических функций, большой параметр - расстояние между стенками. И наконец, мы рассмотрим бильярд в области с малыми рассеивателями, где большим параметром является величина, обратная к размеру рассеивателя. Далее в разделах 5 и 6 мы используем антиинтегрируемый предел для доказательства нескольких хорошо известных (или менее известных) теорем об отслеживании, начиная с описания Л. П. Шильниковым символической динамики около трансверсальной гомоклинической орбиты и заканчивая приложениями к небесной механике. В заключительном разделе 7 мы обсуждаем приложения антиинтегрируемого предела к проблеме диффузии Арнольда.

Многие примеры в этом обзоре классические и восходят к динамическому фольклору, так что мы не всегда даем ссылки на исходные результаты.

\section{2. Стандартное отображение}

Перепишем отображение (1.1) в “лагранжевом виде”. Для этого предположим, что

$$
\left(\begin{array}{l}
x_{-} \\
y_{-}
\end{array}\right) \mapsto\left(\begin{array}{l}
x \\
y
\end{array}\right) \mapsto\left(\begin{array}{l}
x_{+} \\
y_{+}
\end{array}\right) .
$$

Тогда $x_{-}, x, x_{+}$удовлетворяют уравнению

$$
\lambda^{-1}\left(x_{+}-2 x+x_{-}\right)=\sin x .
$$

Стандартное отображение (обозначение: SM), записанное в этом виде, определено на цилиндре $\mathscr{Z}=\mathbb{R}^{2} / \sim$, где отношение эквивалентности $\sim$ следующее:

$$
\left(x_{1}^{\prime}, x_{2}^{\prime}\right) \sim\left(x_{1}, x_{2}\right) \quad \Longleftrightarrow \quad x_{1}^{\prime}-x_{1}=x_{2}^{\prime}-x_{2} \in 2 \pi \mathbb{Z} .
$$

Другими словами, цилиндр $\mathscr{Z}$ является факторпространством плоскости $\mathbb{R}^{2}$ относительно действия группы сдвигов

$$
\left(x_{1}, x_{2}\right) \mapsto\left(x_{1}+2 \pi l, x_{2}+2 \pi l\right), \quad l \in \mathbb{Z} .
$$

Отображение SM действует следующим образом:

$$
\left(x_{-}, x\right) \mapsto \operatorname{SM}\left(x_{-}, x\right)=\left(x, x_{+}\right), \quad \text { где } x_{-}, x, x_{+} \text {удовлетворяют }(2.1) \text {. }
$$


Бесконечные последовательности $\mathbf{x}=\left(x_{k}\right)_{k \in \mathbb{Z}}$ такие, что тройка $\left(x_{-}, x, x_{+}\right)=$ $\left(x_{l-1}, x_{l}, x_{l+1}\right)$ для любого целого $l$ удовлетворяет $(2.1)$, называются траекториями стандартного отображения.

Лагранжева форма стандартного отображения допускает вариационную формулировку. А именно, траектории системы являются критическими точками (экстремалями) формальной суммы

$$
A(\mathbf{x})=\sum_{k \in \mathbb{Z}} L\left(x_{k}, x_{k+1}\right), \quad L\left(x^{\prime}, x^{\prime \prime}\right)=\frac{1}{2 \lambda}\left(x^{\prime}-x^{\prime \prime}\right)^{2}-\cos x^{\prime \prime} .
$$

Это означает, что $\mathbf{x}^{0}=\left(x_{k}^{0}\right)_{k \in \mathbb{Z}}$ является траекторией тогда и только тогда, когда для любого целого $n$

$$
\frac{\partial}{\partial x_{n}} \sum_{k=-\infty}^{\infty} L\left(x_{k}, x_{k+1}\right)=0 \quad \text { в точке } \mathbf{x}=\mathbf{x}^{0} .
$$

В пределе $\lambda \rightarrow \infty$ стандартное отображение теряет динамический смысл, поскольку $x_{+}$нельзя выразить через $x$ и $x_{-}$из равенства $(2.1)$, если $\lambda^{-1}=0$. Однако соответствующая вариационная задача остается корректно определенной. Ее решениями являются последовательности

$$
\mathbf{a}=\left(a_{j}\right)_{j \in \mathbb{Z}}, \quad a_{j} \in \pi \mathbb{Z} .
$$

При больших значениях параметра $\lambda$ стандартное отображение имеет много траекторий, близких к последовательностям (2.6).

Более точно, пусть

$$
\mathscr{A}_{\Lambda}=\left\{\mathbf{a}=\left(a_{k}\right)_{k \in \mathbb{Z}}: a_{k} \in \pi \mathbb{Z},\left|a_{k-1}-2 a_{k}+a_{k+1}\right| \leqslant \Lambda \text { для любого } k \in \mathbb{Z}\right\} .
$$

Для любого $\mathbf{a} \in \mathscr{A}_{\Lambda}$ определим полное метрическое пространство $\Pi=\Pi(\mathbf{a})$ последовательностей

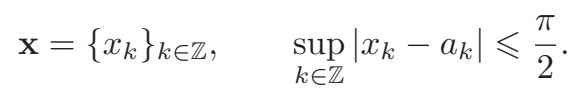

Метрика на П определяется следующим образом:

$$
\rho\left(\mathbf{x}^{\prime}, \mathbf{x}^{\prime \prime}\right)=\sup _{k \in \mathbb{Z}}\left|x_{k}^{\prime}-x_{k}^{\prime \prime}\right|, \quad \mathbf{x}^{\prime}, \mathbf{x}^{\prime \prime} \in \Pi .
$$

Tеорема 1 [6]. Пусть данъь $\Lambda>0, \sigma \in(0, \pi / 2) u$

$$
\lambda_{0}=\lambda_{0}(\Lambda, \sigma)=\max \left\{\frac{\Lambda+4 \sigma}{\sin \sigma}, \frac{8}{\cos \sigma}\right\} .
$$

Для любого $|\lambda| \geqslant \lambda_{0}$ и любого $\mathbf{a} \in \mathscr{A}_{\Lambda}$ стандартное отображение имеет единственную траекторию $\mathbf{x}$ такую, что $\rho(\mathbf{a}, \mathbf{x})<\sigma$.

Последовательности из $\mathscr{A}_{\Lambda}$ можно считать кодами для соответствующих траекторий. Такая возможность кодировать траектории элементами достаточно большого множества типична для хаотических систем. 
Теорема 1 означает, что при больших значениях $\lambda$ имеется инвариантное множество $\mathscr{K}_{\Lambda}=\mathscr{K}_{\Lambda}(\lambda, \sigma) \subset \mathscr{Z}$ такое, что траектории из $\mathscr{K}_{\Lambda}$ находятся во взаимно однозначном соответствии с элементами из $\mathscr{A}_{\Lambda}$. Формальное определение $\mathscr{K}_{\Lambda}-$ следующее. Любой код $\mathbf{a} \in \mathscr{A}_{\Lambda}$ определяет единственную орбиту $\mathbf{x}=\mathbf{x}(\mathbf{a})$. Рассмотрим отображение

$$
\mathscr{A}_{\Lambda} \ni \mathbf{a} \mapsto \zeta(\mathbf{a})=\left(x_{0}, x_{1}\right) / \mathbb{Z} \in \mathscr{Z}
$$

где $\left(x_{0}, x_{1}\right) / \mathbb{Z}$ означает отождествление $(2.2)$. Тогда по определению $\mathscr{K}_{\Lambda}=$ $\zeta\left(\mathscr{A}_{\Lambda}\right)$.

Все траектории в множестве $\mathscr{K}_{\Lambda}$ гиперболичны (см. теорему 2 ниже). Рассмотрев еще один $\mathbb{Z}$-фактор, можно сделать фазовое пространство отображения SM компактным: $\mathscr{Z} / \mathbb{Z}$ диффеоморфно двумерному тору $\mathbb{T}^{2}$. Затем мы докажем, что $K_{\Lambda}=\mathscr{K}_{\Lambda} / \mathbb{Z} \subset \mathbb{T}^{2}$ компактно (теорема 3 ) и, следовательно, гиперболично для факторотображения $\mathrm{SM}_{0}$. Наконец, мы оценим топологическую энтропию соответствующей динамической системы (теорема 4).

ДокАЗАТЕЛЬство теоремы 1. Доказательство основано на принципе сжимающих отображений в метрическом пространстве $(\Pi(\mathbf{a}), \rho)$, где последовательность а $\in \mathscr{A}_{\Lambda}$ зафиксирована. Сначала запишем (2.1) в виде

$$
x_{k}=\arcsin _{k}\left(\frac{x_{k+1}-2 x_{k}+x_{k-1}}{\lambda}\right),
$$

где $\arcsin _{k}-$ ветвь $\sin ^{-1}$ такая, что $\arcsin _{k} 0=a_{k}$. Таким образом, $\arcsin _{k}$ отображает интервал $(-1,1)$ в интервал $\left(a_{k}-\pi / 2, a_{k}+\pi / 2\right)$. Другими словами, $\arcsin _{k} x=a_{k} \pm \arcsin x$, где $\arcsin =\arcsin _{0}:(-1,1) \rightarrow(-\pi / 2, \pi / 2)-$ стандартная ветвь $\sin ^{-1}$ и знак $+(-)$ берется, когда $a_{k} / \pi$ четное (нечетное). Последовательность $\mathbf{x}=\mathbf{a}$ является траекторией уравнений $(2.8)$ при $\lambda=\infty$.

Рассмотрим отображение $\mathbf{x} \mapsto \mathbf{y}=\Phi(\mathbf{x})$ такое, что

$$
y_{k}=\arcsin _{k}\left(\frac{x_{k+1}-2 x_{k}+x_{k-1}}{\lambda}\right) .
$$

Тогда любая неподвижная точка оператора $\Phi$ является траекторией SM.

Пусть $B_{\sigma}(\mathbf{a}) \subset \Pi$ - замкнутый шар с центром а и радиусом $\sigma$.

ЛЕмма 1. Пусть $|\lambda|>\lambda_{0}$. Тогда

1) $\Phi$ определен на $B=B_{\sigma}(\mathbf{a}) u \Phi(B) \subset B$;

2) $\Phi$ является сжимающим на $B$, т.е.

$$
\rho\left(\Phi\left(\mathbf{x}^{\prime}\right), \Phi\left(\mathbf{x}^{\prime \prime}\right)\right)<\frac{1}{2} \rho\left(\mathbf{x}^{\prime}, \mathbf{x}^{\prime \prime}\right) \quad \text { для всех } \mathbf{x}^{\prime}, \mathbf{x}^{\prime \prime} \in B .
$$

Согласно принципу сжимающих отображений теорема 1 следует из леммы 1. Обратимся к доказательству леммы. Напомним, что $\sigma<\pi / 2$. Чтобы проверить включение $\Phi(B) \subset B$, достаточно показать, что для любого $\mathbf{x} \in B$

$$
\left|\frac{x_{k+1}-2 x_{k}+x_{k-1}}{\lambda}\right|<\sin \sigma .
$$


Так как $\rho(\mathbf{x}, \mathbf{a})<\sigma$, имеем:

$$
\left|x_{k+1}-2 x_{k}+x_{k-1}\right| \leqslant \Lambda+4 \sigma .
$$

Следовательно, неравенство (2.10) выполнено при

$$
\lambda_{0}>\frac{\Lambda+4 \sigma}{\sin \sigma} .
$$

Отметим, что для любой пары вещественных чисел $u^{\prime}, u^{\prime \prime} \in(-\sin \sigma, \sin \sigma)$

$$
\left|\arcsin u^{\prime}-\arcsin u^{\prime \prime}\right| \leqslant \frac{1}{\cos \sigma}\left|u^{\prime}-u^{\prime \prime}\right| .
$$

Здесь

$$
\frac{1}{\cos \sigma}=\sup _{u \in(-\sin \sigma, \sin \sigma)}\left|\frac{d}{d u} \arcsin u\right| .
$$

Положим $\mathbf{y}^{\prime}=\Phi\left(\mathbf{x}^{\prime}\right), \mathbf{y}^{\prime \prime}=\Phi\left(\mathbf{x}^{\prime \prime}\right)$. Тогда для любого $k \in \mathbb{Z}$ имеем:

$$
\begin{aligned}
\left|y_{k}^{\prime}-y_{k}^{\prime \prime}\right| & =\left|\arcsin \left(\frac{x_{k+1}^{\prime}-2 x_{k}^{\prime}+x_{k-1}^{\prime}}{\lambda}\right)-\arcsin \left(\frac{x_{k+1}^{\prime \prime}-2 x_{k}^{\prime \prime}+x_{k-1}^{\prime \prime}}{\lambda}\right)\right| \\
& \leqslant \frac{1}{\cos \sigma}\left|\frac{x_{k+1}^{\prime}-2 x_{k}^{\prime}+x_{k-1}^{\prime}}{\lambda}-\frac{x_{k+1}^{\prime \prime}-2 x_{k}^{\prime \prime}+x_{k-1}^{\prime \prime}}{\lambda}\right| \\
& \leqslant \frac{\left|x_{k+1}^{\prime}-x_{k+1}^{\prime \prime}\right|+2\left|x_{k}^{\prime}-x_{k}^{\prime \prime}\right|+\left|x_{k-1}^{\prime}-x_{k-1}^{\prime \prime}\right|}{\lambda \cos \sigma} \\
& \leqslant \frac{4}{\lambda \cos \sigma} \rho\left(\mathbf{x}^{\prime}, \mathbf{x}^{\prime \prime}\right) .
\end{aligned}
$$

Таким образом, неравенство (2.9) выполнено, если $\lambda_{0}>8 / \cos \sigma$. Лемма доказана.

Теорема 2. Пустъ даны $\Lambda>0, \sigma \in(0, \pi / 2) u \lambda$, где $|\lambda|>\lambda_{0}(\Lambda, \sigma)$. Тогда любая орбита $\mathbf{x}(\mathbf{a}), \mathbf{a} \in \mathscr{A}_{\Lambda}$, гиперболична.

ДокАзАтельство. Напомним, что стандартное определение гиперболической орбиты $\mathbf{x}=\mathbf{x}(\mathbf{a})$ дается в терминах инвариантного разложения касательных пространств в любой точке на растягивающееся и сжимающееся подпространства [52]. Вместо этого мы используем конический критерий гиперболичности В. М. Алексеева, также содержащийся в [52].

В этом доказательстве для краткости мы используем обозначение $f=\mathrm{SM}$. Согласно (2.1) матрица Якоби отображения $f$ имеет вид

$$
D f\left(x_{-}, x\right)=\left(\begin{array}{cc}
0 & 1 \\
-1 & 2+\lambda \cos x
\end{array}\right) .
$$

В каждой точке $q=\left(x_{-}, x\right) \in \mathscr{K}_{\Lambda}$ определим конусы

$$
\begin{aligned}
H_{q} & =\left\{\left(u_{-}, u\right) \in T_{q} \mathscr{Z}:\left\|u_{-}\right\| \leqslant \alpha_{H}\|u\|\right\}, \\
V_{q} & =\left\{\left(u_{-}, u\right) \in T_{q} \mathscr{Z}:\|u\| \leqslant \alpha_{V}\left\|u_{-}\right\|\right\},
\end{aligned}
$$

где $\left(u_{-}, u\right)$ - координаты на $T_{q} \mathscr{Z}$, индуцированные координатами $\left(x_{-}, x\right)$ на $\mathscr{Z}$. 
Согласно коническому критерию, чтобы доказать гиперболичность траекторий в инвариантном множестве $\mathscr{K}_{\Lambda}$, достаточно проверить, что существует $\mu>1$ такое, что при всех $q \in \mathscr{K}_{\Lambda}$ выполнено следующее:

$$
\begin{gathered}
D f_{q} H_{q} \subset \operatorname{Int} H_{f(q)}, \quad D f_{q}^{-1} V_{f(q)} \subset \operatorname{Int} V_{q}, \\
\left\|D f_{q} \xi\right\| \geqslant \mu\|\xi\| \quad \text { при } \xi \in H_{q}, \quad\left\|D f_{q}^{-1} \xi\right\| \geqslant \mu\|\xi\| \quad \text { при } \xi \in V_{f(q)} .
\end{gathered}
$$

Возьмем $\alpha_{H}=\alpha_{V}=1 / 2$. Для любого $q \in \mathscr{K}_{\Lambda}(\lambda, \sigma)$ и $\left(u_{-}, u\right) \in H_{q}$ имеем:

$$
D f(q)\left(\begin{array}{c}
u_{-} \\
u
\end{array}\right)=\left(\begin{array}{c}
u \\
u_{+}
\end{array}\right), \quad u_{+}=-u_{-}+(2+\lambda \cos x) u .
$$

Мы видим, что

$$
\frac{|u|}{\left|u_{+}\right|} \leqslant \frac{1}{\lambda \cos \sigma-5 / 2} .
$$

Следовательно,

$$
\left(\begin{array}{c}
u \\
u_{+}
\end{array}\right) \in \operatorname{Int} H_{f(q)}, \quad \text { если } \quad \lambda>\frac{3}{\cos \sigma} .
$$

Отсюда вытекает первое включение в (2.13). Имеем также

$$
\left\|\left(\begin{array}{c}
u \\
u_{+}
\end{array}\right)\right\|^{2}=u^{2}+\left(\lambda \cos x-\frac{5}{2}\right)^{2} u^{2} \geqslant 4\left\|\left(\begin{array}{c}
u_{-} \\
u
\end{array}\right)\right\|^{2},
$$

если $\lambda>9 /(2 \cos \sigma)$. Таким образом, первое неравенство в $(2.14)$ выполнено c $\mu=2$.

Второе включение в (2.13) и второе неравенство в (2.14) проверяются аналогично. Остается заметить, что $\lambda_{0}>9 /(2 \cos \sigma)$. Теорема доказана.

Чтобы придать хаотическим свойствам отображения SM количественный характер, оценим его топологическую энтропию. Поскольку в случае некомпактного фазового пространства топологическая энтропия обычно бесконечна, мы перейдем к факторсистеме с компактным фазовым пространством. Для этого отметим, что равенства (1.1) можно рассматривать по модулю $2 \pi$. Это порождает факторсистему

$$
\mathrm{SM}_{0}: \mathbb{T}^{2} \rightarrow \mathbb{T}^{2}, \quad \mathbb{T}^{2}=\mathbb{R}^{2} /\left(2 \pi \mathbb{Z}^{2}\right),
$$

такую, что диаграмма

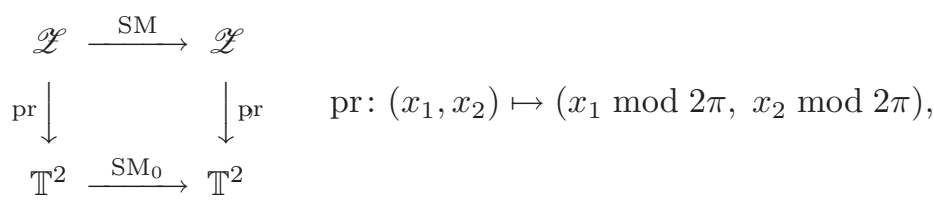

коммутативна.

Рассмотрим на $\mathscr{A}_{\Lambda}$ и на пространстве $\mathscr{O}$ орбит отображения SM следующее действие группы $\mathbb{Z}$ : для любых $\left(l_{1}, l_{2}\right) \in \mathbb{Z}^{2}$

$$
\begin{aligned}
\mathbf{a} & =\left(a_{i}\right)_{i \in \mathbb{Z}} \mapsto l(\mathbf{a})=\left(a_{i}+2 \pi l_{1}+2 \pi i l_{2}\right)_{i \in \mathbb{Z}}, \\
\mathscr{O} \ni \mathbf{x} & =\left(x_{i}\right)_{i \in \mathbb{Z}} \mapsto l(\mathbf{x})=\left(x_{i}+2 \pi l_{1}+2 \pi i l_{2}\right)_{i \in \mathbb{Z}} .
\end{aligned}
$$


Определим факторпространства

$$
A_{\Lambda}=\mathscr{A}_{\Lambda} / \mathbb{Z}^{2}, \quad O=\mathscr{O} / \mathbb{Z}^{2}, \quad K_{\Lambda}=\operatorname{pr} \mathscr{K}_{\Lambda} .
$$

Тогда $A_{\Lambda}$ может считаться пространством кодов, а $O$ - соответствующим пространством орбит для $\mathrm{SM}_{0}$. Пространство $A_{\Lambda}$ можно отождествить с

$$
B_{\Lambda}=\left\{\mathbf{b}=\left(b_{i}\right)_{i \in \mathbb{Z}}: b_{i} \in \pi \mathbb{Z},\left|b_{i}\right| \leqslant \Lambda\right\}
$$

при помощи биекции

$$
\mathbf{a}=\left(a_{i}\right)_{i \in \mathbb{Z}} \mapsto \mathbf{b}=\left(b_{i}\right)_{i \in \mathbb{Z}}, \quad b_{i}=a_{i-1}-2 a_{i}+a_{i+1},
$$

уважающей оператор сдвига $\mathscr{T}:\left(a_{i}\right)_{i \in \mathbb{Z}} \mapsto\left(a_{i+1}\right)_{i \in \mathbb{Z}}$.

Напомним определение сдвига Бернулли. Пусть $J$ - конечное множество, состоящее из $q$ символов, а $\Sigma_{q}=J^{\mathbb{Z}}$ - множество последовательностей $\mathbf{a}=\left(a_{i}\right)_{i \in \mathbb{Z}}$ элементов из $J$. Наделим $\Sigma_{q}$ (тихоновской) топологией прямого произведения: база состоит из цилиндров

$$
U_{I}(\mathbf{a})=\left\{\mathbf{a}^{\prime}: a_{i}^{\prime}=a_{i} \text { при } i \notin I\right\}, \quad \text { где } I \subset \mathbb{Z} \text { - конечное множество. }
$$

Хорошо известно [52], что $\Sigma_{q}$ - компактное вполне несвязное пространство без изолированных точек и, следовательно, гомеоморфно стандартному канторову множеству. Сдвиг Бернулли с $q$ символами - это сдвиг $\mathscr{T}: \Sigma_{q} \rightarrow \Sigma_{q}$.

Пусть

$$
q=\#(\pi \mathbb{Z} \cap[-\Lambda, \Lambda])=1+2\left[\frac{\Lambda}{\pi}\right],
$$

где [·] - целая часть вещественного числа.

ТЕОрема 3. Для любого $\lambda>\lambda_{0}$ множество $K_{\Lambda}$ является компактным гиперболическим инвариантным множеством для стандартного отображения u $\mathrm{SM}_{0}: K_{\Lambda} \rightarrow K_{\Lambda}$ топологически сопряюсено сдвигу Бернулли на пространстве из q символов.

ДокАЗАтельство. Требуется только показать, что $\zeta: A_{\Lambda} \rightarrow K_{\Lambda}$ непрерывно. Топология прямого произведения на $B_{\Lambda}$ имеет базу открытых множеств (2.15), где $J=B_{\Lambda}$. В частности, для любого а $\in B_{\Lambda}$ цилиндр

$$
C_{n}(\mathbf{a})=\left\{\mathbf{a}^{\prime} \in B_{\Lambda}: a_{i}^{\prime}=a_{i} \text { при }|i| \leqslant n\right\}
$$

- открытое множество, содержащее а. Непрерывность $\zeta$ следует из непрерывности отображения $\mathbf{a} \mapsto \mathbf{x}(\mathbf{a})$, которая вытекает из следующей леммы.

Лемма 2. Предположим, что $|\lambda| \geqslant \lambda_{0} u \mathbf{a}^{\prime} \in C_{n}(\mathbf{a})$. Тогда орбиты $\mathbf{x}=\mathbf{x}(\mathbf{a})$ $u \mathbf{x}^{\prime}=\mathbf{x}\left(\mathbf{a}^{\prime}\right)$ удовлетворяют оценкам

$$
\left|x_{k}^{\prime}-x_{k}\right| \leqslant 5^{|k|-n} \cdot 2 \sigma \quad \text { для любого }|k| \leqslant n .
$$

ДокАЗАТЕЛЬСТво лЕммы 2. Мы получим последовательности $\mathbf{x}$ и $\mathbf{x}^{\prime}$ как пределы

$$
\begin{gathered}
\mathbf{x}=\lim _{j \rightarrow \infty} \mathbf{x}^{(j)}, \quad \mathbf{x}^{\prime}=\lim _{j \rightarrow \infty} \mathbf{x}^{\prime(j)}, \\
\mathbf{x}^{(0)}=\mathbf{a}, \quad \mathbf{x}^{\prime(0)}=\mathbf{a}^{\prime}, \quad \mathbf{x}^{(j+1)}=\Phi \mathbf{x}^{(j)}, \quad \mathbf{x}^{\prime(j+1)}=\Phi \mathbf{x}^{\prime(j)},
\end{gathered}
$$

где $\Phi$ - оператор из леммы 1 . 
Используем индукцию по $j$. Для $j=0$ неравенства (2.17) выполнены, поскольку $x_{k}^{(0)}=x_{k}^{\prime(0)}$ при $|k| \leqslant n$. Если $k=n$, то $(2.17)$ при всех $j>0$ следует из утверждения 1) леммы 1.

Предположим, что (2.17) выполнено для некоторого $j=s$. Чтобы доказать его для $j=s+1$, используем оценку

$$
\left|\mathbf{x}_{k}^{(j+1)}-\mathbf{x}_{k}^{(j+1)}\right| \leqslant \frac{\left|\mathbf{x}_{k+1}^{(j)}-\mathbf{x}_{k+1}^{\prime(j)}\right|+2\left|\mathbf{x}_{k}^{(j)}-\mathbf{x}_{k}^{\prime(j)}\right|+\left|\mathbf{x}_{k-1}^{(j)}-\mathbf{x}_{k-1}^{\prime(j)}\right|}{\lambda \cos \sigma}
$$

которая следует из (2.11). Для любого $|k| \leqslant n-1$ по предположению индукции имеем:

$$
\left|\mathbf{x}_{k}^{(s+1)}-\mathbf{x}_{k}^{\prime(s+1)}\right| \leqslant \frac{\left(5^{-n+|k|+1}+2 \cdot 5^{-n+|k|}+5^{-n+|k|-1}\right) \cdot 2 \sigma}{\lambda \cos \sigma} .
$$

Правая часть этого неравенства не превосходит $5^{-n+|k|} \cdot 2 \sigma$, поскольку ввиду (2.7) $\lambda_{0} \cos \sigma \geqslant 8$. Лемма доказана.

В [45] имеется другое доказательство похожей оценки.

Теорема 4. Существует постоянная $c>0$ такая, что для достаточно больших $\lambda$ топологическая энтропия отобраюения $\mathrm{SM}_{0}$ допускает оценку ${ }^{2}$

$$
h_{\text {top }}\left(\mathrm{SM}_{0}\right) \geqslant c \log \lambda \text {. }
$$

ДоказАтельство. Мы уже доказали, что для достаточно больших $\lambda$ отображение $\mathrm{SM}_{0}$ имеет инвариантное множество $K_{\Lambda}$ такое, что ограничение $\left.\mathrm{SM}_{0}\right|_{K_{\Lambda}}$ сопряжено сдвигу Бернулли $\mathscr{T}: \Sigma_{q} \rightarrow \Sigma_{q}$ на пространстве из $q$ символов, определенных в $(2.16)$. Следовательно, $h_{\text {top }}\left(\mathrm{SM}_{0}\right)$ не меньше, чем топологическая энтропия $h_{q}$ сдвига Бернулли [52]. Величину $h_{q}$ можно вычислить, например, из следующего равенства [75] (которое также выполняется для топологических цепей Маркова):

$$
h_{q}=\lim _{n \rightarrow \infty} \frac{1}{n} \log \theta_{n},
$$

где $\theta_{n}$ - число элементов в множестве всех допустимых $n$-последовательностей. В данном случае $\theta_{n}=q^{n}$. Следовательно, $h_{q}=\log q$.

Остается заметить, что согласно (2.7) величину $\Lambda$ можно выбрать большей, чем произведение положительной постоянной и $\lambda$. Теорема доказана.

\section{3. Дискретные лагранжевы системы}

Антиинтегрируемый предел обычно обсуждается в лагранжевых системах. Мы сконцентрируемся на лагранжевых системах с дискретным временем, которые называются дискретными лагранжевыми системами (ДЛС). Случай непрерывного времени будет сводиться к случаю дискретного времени (см. раздел 6).

\footnotetext{
2 Явные оценки для $c$ и минимального $\lambda$ можно легко получить из доказательства (см. также [54]). Однако вряд ли стоит надеяться на то, что эти оценки будут близки к оптимальным.
} 
3.1. Простейший случай. Пусть $M-m$-мерное многообразие и $L-$ гладкая ${ }^{3}$ функция на $M^{2}=M \times M$. По определению последовательность $\mathbf{x}=$ $\left(x_{i}\right)_{i \in \mathbb{Z}}, x_{i} \in M$, является траекторией ДЛС с лагранжианом $L$, если $\mathbf{x}-$ экстремаль функционала действия, имеющего вид формальной суммы

$$
A(\mathbf{x})=\sum L\left(x_{i}, x_{i+1}\right)
$$

в том же смысле, что и в случае стандартного отображения (см. (2.5)). Эквивалентно, для любого $i \in \mathbb{Z}$

$$
\partial_{x_{i}}\left(L\left(x_{i-1}, x_{i}\right)+L\left(x_{i}, x_{i+1}\right)\right)=0 .
$$

Траектории не меняются при умножении лагранжиана на постоянную, при добавлении постоянной к $L$ и при калибровочном преобразовании

$$
L(x, y) \mapsto L(x, y)+f(x)-f(y)
$$

с произвольной функцией $f$ на $M$. Калибровочное преобразование не меняет функционал действия.

Если уравнение

$$
\partial_{x}\left(L\left(x_{-}, x\right)+L\left(x, x_{+}\right)\right)=0
$$

можно глобально разрешить относительно $x_{-}=x_{-}\left(x, x_{+}\right)$, а также относительно $x_{+}=x_{+}\left(x_{-}, x\right)$, то отображение

$$
\left(x_{-}, x\right) \mapsto T\left(x_{-}, x\right)=\left(x, x_{+}\right)
$$

является диффеоморфизмом и определяет дискретную динамическую систему на $M \times M$. Однако обычно отображение $T$ определено лишь локально.

Чтобы указать условия локального существования и гладкости отображения $T$, определим ${ }^{4}$

$$
B(x, y)=\partial_{x} \partial_{y} L(x, y)
$$

или, в локальных координатах,

$$
B(x, y)=\left(\frac{\partial^{2} L}{\partial x_{i} \partial y_{j}}\right) .
$$

В инвариантных терминах $B(x, y)$ - линейный оператор $T_{x} M \rightarrow T_{y}^{*} M$ или билинейная форма на $T_{x} M \times T_{y} M$. Будем говорить, что лагранжиан $L$ закручивающий, если он удовлетворяет следующему условию.

УСлОвИЕ ЗАКРУчИвАния. $B(x, y)$ невырожден при всех $x, y \in M$.

В этом случае отображение $T$ локально определено и является гладким. Легко проверить (см., например, [74]), что $T$ симплектично относительно симплектической 2-формы $\omega=B(x, y) d x \wedge d y$,

$$
\omega(u, v)=\left\langle B(x, y) u_{1}, v_{2}\right\rangle-\left\langle B(x, y) v_{1}, u_{2}\right\rangle, \quad u=\left(u_{1}, u_{2}\right), \quad v=\left(v_{1}, v_{2}\right) .
$$

\footnotetext{
${ }^{3} C^{3}$ более чем достаточно.

${ }^{4}$ Иногда оператор $B$ определеляется как $-\partial_{x} \partial_{y} L$, тогда для многих естественных ДЛС он оказывается положительно определенным.
} 
Если выполнено условие закручивания, то преобразование Лежандра $S$ : $M^{2} \rightarrow T^{*} M$, действующее по правилу

$$
(x, y) \mapsto\left(x, p_{x}\right), \quad p_{x}=-\partial_{x} L(x, y),
$$

локально обратимо и $T$ можно представить как локально определенное отображение $F=S T S^{-1}: T^{*} M \rightarrow T^{*} M$. Отображение $F$ является симплектическим относительно стандартной симплектической формы $d p_{x} \wedge d x$ на $T^{*} M$, и $L-$ производящая функция для $F$ :

$$
F\left(x, p_{x}\right)=\left(y, p_{y}\right) \Longleftrightarrow p_{x}=-\partial_{x} L(x, y), \quad p_{y}=\partial_{y} L(x, y) .
$$

Такое симплектическое отображение $F$ называется закручивающим (см. [47]). Обычно оно определено лишь локально.

Условие закручивания накладывает на $M$ сильные топологические ограничения, оно редко выполнено на всем $M \times M$. Вот два канонических примера ДЛС.

1. Многомерное стандартное отображение:

$$
L(x, y)=\frac{1}{2}\langle B(x-y), x-y\rangle-\frac{1}{2}(V(x)+W(y)), \quad x, y \in \mathbb{R}^{m},
$$

где $B$ - симметрическая постоянная невырожденная матрица. В этом случае $L$ определяет симплектическое закручивающее отображение $F: \mathbb{R}^{2 m} \rightarrow \mathbb{R}^{2 m}$.

Обычно $V$ предполагается $\mathbb{Z}^{m}$-периодическим. Тогда фазовое пространство $\mathbb{R}^{2 m}$ можно профакторизовать относительно $\mathbb{Z}^{m}$-действия $(x, y) \mapsto(x+k$, $y+k), k \in \mathbb{Z}^{m}$, и $F$ оказывается симплектическим отображением $\mathbb{T}^{m} \times \mathbb{R}^{m}$ на себя.

Более подробно мы обсудим такие лагранжианы в п. 4.3.

2. Рассмотрим область $D \subset \mathbb{R}^{m}$, ограниченную гладкой выпуклой гиперповерхностью $M$. Бильярдная система в $D$ - это ДЛС с лагранжианом $L(x, y)=$ $|x-y|$ на $M \times M$. Проверим, что $L$ удовлетворяет условию закручивания на $(M \times M) \backslash \Delta$, где $\Delta=\{(x, x): x \in M\}$. Пусть $\langle B(x, y) v, w\rangle$ - билинейная форма на $\mathbb{R}^{m} \times \mathbb{R}^{m}$, соответствующая оператору $B(x, y)=\partial_{x} \partial_{y} L(x, y)$. В результате прямого вычисления получаем:

$$
\langle B(x, y) v, w\rangle=\frac{-\langle v, w\rangle+\langle v, e\rangle\langle w, e\rangle}{|x-y|}, \quad e=\frac{x-y}{|x-y|} .
$$

Очевидно, что $e$ лежит и в левом, и в правом ядре: $B(x, y) e=B^{*}(x, y) e=0$. Поэтому если $e \notin T_{x} M$ и $e \notin T_{y} M$, то ограничение билинейной формы $B(x, y)$ на $T_{x} M \times T_{y} M$ невырождено и, следовательно, $L$ - закручивающий лагранжиан. Это всегда выполнено, если граница строго выпукла. Все это хорошо известно, среди относительно недавних ссылок можно назвать [12].

На самом деле можно отождествить $T_{x} M$ и $T_{y} M$ с помощью изоморфизма $\Pi(x, y): T_{x} M \rightarrow T_{y} M$, являющегося параллельной проекцией в $\mathbb{R}^{m}$ вдоль отрезка $[x, y]: \Pi v=v \bmod e$. Тогда

$$
\langle B(x, y) v, \Pi(x, y) v\rangle=\frac{-|v|^{2}+\langle v, e\rangle^{2}}{|x-y|}<0, \quad v \in T_{x} M \backslash\{0\} .
$$


Зададим ориентацию на $M$ как на границе. Поскольку П $(x, y)$ меняет ориентацию, получаем, что $(-1)^{m} \operatorname{det} B(x, y)>0$. Отметим, что так как образ и область определения оператора $B(x, y)$ различны, то $\operatorname{det} B(x, y)$ - не определен инвариантно (во всяком случае, как число), но знак определителя определен.

Если область $D$ невыпукла, то бильярдное отображение $T$ определено на

$$
\{(x, y) \in(M \times M) \backslash \Delta \text { : отрезок }(x, y) \text { содержится в } D\},
$$

и имеет особенности, когда отрезок $(x, y)$ касается $M$.

В [74] читатель может найти другие (в основном, интегрируемые) примеры ДЛС, включая многозначные.

3.2. Многозначные лагранжианы. В приложениях дискретный лагранжиан $L$ в ДЛС с конфигурационным пространством $M$ обычно многозначен. Часто бывает так, что $L$ - функция на универсальной накрывающей для $M \times M$. Например, для стандартного отображения можно считать, что $M=\mathbb{T}^{m}$ и $L-$ функция на $\mathbb{R}^{2 m}$. О таком $L$ можно думать как о многозначной функции, т. е. о наборе функций на открытых множествах в $M \times M$. Чтобы обслужить большую часть приложений, мы используем следующее определение ДлС.

ДЛС с $m$ степенями свободы определяется конечным или счетным набором $\mathscr{L}=\left\{L_{\kappa}\right\}_{\kappa \in J}$ функций (лагранжианов) на $m$-мерных многообразиях $U_{\kappa}^{-} \times U_{\kappa}^{+}$ (обычно $U_{\kappa}^{ \pm}$- открытые множества на конфигурационном пространстве $M$ ). Траектория ДЛС $\mathscr{L}$, соответствующая коду $\mathbf{k}=\left(\kappa_{i}\right)_{i \in \mathbb{Z}},-$ это последовательность

$$
\mathbf{x}=\left(x_{i}\right)_{i \in \mathbb{Z}}, \quad x_{i} \in U_{\kappa_{i-1}}^{+} \cap U_{\kappa_{i}}^{-},
$$

являющаяся критической точкой формального дискретного функционала действия

$$
A_{\mathbf{k}}(\mathbf{x})=\sum_{i \in \mathbb{Z}} L_{\kappa_{i}}\left(x_{i}, x_{i+1}\right), \quad \mathbf{k}=\left(\kappa_{i}\right)_{i \in \mathbb{Z}}
$$

Более точно,

$$
\partial_{x_{i}}\left(L_{\kappa_{i-1}}\left(x_{i-1}, x_{i}\right)+L_{\kappa_{i}}\left(x_{i}, x_{i+1}\right)\right)=0, \quad i \in \mathbb{Z} .
$$

Таким образом, траектория - это пара $(\mathbf{k}, \mathbf{x})$. Код $\mathbf{k}$ можно считать путем на ориентированном графе с множеством вершин $J$. Вершины $\kappa, \kappa^{\prime} \in J$ соединены ребром $\gamma=\left(\kappa, \kappa^{\prime}\right)$, если $U_{\kappa}^{-} \cap U_{\kappa^{\prime}}^{+} \neq \varnothing$.

В этой статье мы не используем глобальные методы, поэтому, выбирая локальные координаты, мы можем считать, что $U_{\kappa}^{ \pm}$- открытые множества в $\mathbb{R}^{m}$. Если лагранжианы удовлетворяют условию закручивания

$$
\operatorname{det} B_{\kappa}(x, y) \neq 0, \quad B_{\kappa}(x, y)=\partial_{x} \partial_{y} L_{\kappa}(x, y),
$$

то ребро $\gamma=\left(\kappa, \kappa^{\prime}\right)$ определяет локальный диффеоморфизм

$$
f_{\gamma}: \mathscr{D}_{\gamma}^{-} \rightarrow \mathscr{D}_{\gamma}^{+}
$$

открытых множеств $\mathscr{D}_{\gamma}^{ \pm}$в $\mathbb{R}^{2 m}$ с помощью равенства

$$
f_{\gamma}(x, y)=(y, z) \Longleftrightarrow \partial_{y}\left(L_{\kappa}(x, y)+L_{\kappa^{\prime}}(y, z)\right)=0 .
$$


Тогда траектория $(\mathbf{k}, \mathbf{x})$ соответствует композиции

$$
\left(x_{n}, x_{n+1}\right)=f_{\gamma_{n}} \circ f_{\gamma_{n-1}} \circ \cdots \circ f_{\gamma_{1}}\left(x_{0}, x_{1}\right), \quad \gamma_{i}=\left(\kappa_{i}, \kappa_{i-1}\right) .
$$

Такую динамику принято представлять одним отображением, перейдя к косому произведению [52] отображений $\left\{f_{\gamma}\right\}$. Но часто этого не требуется: динамику можно представить единым отображением гладкого многообразия $P$ на себя. Мы говорим, что динамическая система $F: P \rightarrow P$ является объемлющей для ДЛС $\mathscr{L}$, если каждой траектории $(\mathbf{k}, \mathbf{x})$ в $\mathscr{L}$ соответствует траектория $\mathbf{z}=$ $\left(z_{i}\right)_{i \in \mathbb{Z}}$ в $F$. Дадим формальное определение.

ОПРедЕлениЕ 1. Динамическая система $F: P \rightarrow P$ называется объемлющей для ДЛС $\mathscr{L}$, если существуют две системы открытых множеств $\mathscr{P} \underset{\gamma}{ \pm} P$ и диффеоморфизмы $\beta_{\gamma}^{ \pm}: \mathscr{D}_{\gamma}^{ \pm} \rightarrow \mathscr{P}_{\gamma}^{ \pm}$, которые сопрягают $f_{\gamma}$ с $F$, другими словами, если коммутативна следующая диаграмма:

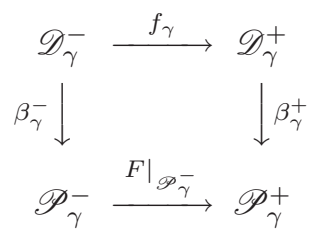

Таким образом, $\beta_{\gamma}^{ \pm}$- карты на $P$, а $f_{\gamma}$ - координатное представление для $F$. Объемлющая система существует, если наложить на ДЛС некоторые слабые условия, но, вообще говоря, она не единственна. Для глобально определенной ДЛС из п. 3.1 объемлющей системой является отображение $T: M \times M \rightarrow$ $M \times M$. Задача построения объемлющей системы не важна для наших целей, поскольку обычно мы начинаем с динамической системы $F: P \rightarrow P$ и соответствующая ДЛС $\mathscr{L}$ является техническим средством для изучения динамики $F$. Стандартным примером является лагранжево представление симплектического отображения из следующего пункта.

3.3. ДЛС, порожденная симплектическим отображением. ДЛС определяет (вообще говоря, локальное) симплектическое отображение. Обратно, можно представить динамику симплектического отображения с помощью ДЛС, но лагранжиан будет определен лишь локально. Пусть

$$
F: P \rightarrow P, \quad F^{*} \omega=\omega
$$

- симплектическое отображение симплектического $2 m$-мерного многообразия $(P, \omega)$ на себя [4].

Пусть $\left(x^{-}, y^{-}\right)$и $\left(x^{+}, y^{+}\right)$- симплектические координаты на малых окрестностях $D^{-}$и $D^{+}$точек $z^{-}$и $z^{+}=F\left(z^{-}\right)$:

$$
\left.\omega\right|_{D^{-}}=d y^{-} \wedge d x^{-},\left.\quad \omega\right|_{D^{+}}=d y^{+} \wedge d x^{+} .
$$

Если $F\left(D^{-}\right) \cap D^{+} \neq \varnothing$, то отображение $F$ можно представить в виде

$$
y^{+}=y^{+}\left(x^{-}, y^{-}\right), \quad x^{+}=x^{+}\left(x^{-}, y^{-}\right), \quad d y_{+} \wedge d x_{+}=d y_{-} \wedge d x_{-} .
$$


Координаты можно выбрать так, что $\operatorname{det}\left(\partial y^{+} / \partial x^{-}\right) \neq 0$. Тогда $F: D^{-} \rightarrow D^{+}$ локально определено производящей функцией $L\left(x^{-}, x^{+}\right)$на открытом множестве $U^{-} \times U^{+}$:

$$
y^{-}=\frac{\partial L}{\partial x^{-}}, \quad y^{+}=-\frac{\partial L}{\partial x^{+}} .
$$

Поскольку конструкция локальная, получаем набор $\mathscr{L}=\left\{L_{\kappa}\right\}_{\kappa \in J}$ производящих функций, определенных на открытых множествах $U_{\kappa}^{-} \times U_{\kappa}^{+}$. Более точно, возьмем набор симплектических карт на $P$, т. е. открытых множеств $\left\{D_{k}\right\}_{k \in I}$ и симплектических отображений

$$
\phi_{k}: D_{k} \rightarrow U_{k} \times \mathbb{R}^{m}, \quad U_{k} \subset \mathbb{R}^{m}, \quad \phi_{k}^{*}(d y \wedge d x)=\left.\omega\right|_{D_{k}} .
$$

Пусть $J$ - множество $\kappa=\left(\kappa_{-}, \kappa_{+}\right) \in I^{2}$ таких, что $F\left(D_{\kappa_{-}}\right) \cap D_{\kappa_{+}} \neq \varnothing$. При любом $\kappa \in J$ отображение $F: D_{\kappa_{-}} \cap F^{-1}\left(D_{\kappa_{+}}\right) \rightarrow F\left(D_{\kappa_{-}}\right) \cap D_{\kappa_{+}}$таково, что

$$
F\left(x^{-}, y^{-}\right)=\left(x^{+}, y^{+}\right) \Longleftrightarrow y^{+} d x^{+}-y^{-} d x^{-}=d L_{\kappa}
$$

для некоторой функции $L_{\kappa}$. Заменив, если требуется, координаты, можно локально выразить $L_{\kappa}$ как гладкую функцию $L_{\kappa}\left(x^{-}, x^{+}\right)$на некотором подмножестве в $U_{\kappa_{-}} \times U_{\kappa_{+}}$. Получаем ДЛС $\mathscr{L}=\left\{L_{\kappa}\right\}_{\kappa \in J}$. Соответствующий граф имеет в качестве множества вершин $J$, и имеется ребро из $\kappa$ в $\kappa^{\prime} \in J$, если $\kappa_{+}=\kappa_{-}^{\prime}$.

Орбита $\mathbf{z}=\left(z_{i}\right)$ отображения $F$ такая, что $z_{i} \in D_{k_{i}}$ при всех $i$, соответствует траектории $(\mathbf{k}, \mathbf{x})$ в ДЛС. При этом $x_{i} \in U_{k_{i}}$ и $\kappa_{i}=\left(k_{i}, k_{i+1}\right)$. Любая траектория $\mathscr{L}$ дает единственную траекторию $F$.

Если карты $\left\{D_{k}\right\}_{k \in I}$ покрывают $P$, то любая траектория $F$ соответствует некоторой (возможно, не единственной) траектории ДЛС $\mathscr{L}$. В большинстве наших примеров ниже $\bigcup_{k \in I} D_{k} \neq P$.

Отображение $F: P \rightarrow P$ определяет объемлющую систему для ДЛС $\mathscr{L}$. Конкретный пример приведен в п. 5.4.

ЗАмЕчАНИЕ 1. Имеются и другие способы представлять траектории симплектического отображения $F$ с помощью ДЛС. Если уравнения (3.12) можно разрешить в виде $y^{-}\left(x^{-}, y^{+}\right)$, то $F: D^{-} \rightarrow D^{+}$локально представляется производящей функцией $S\left(x^{-}, y^{+}\right)$:

$$
F\left(x^{-}, y^{-}\right)=\left(x^{+}, y^{+}\right) \Longleftrightarrow d S\left(x^{-}, y^{+}\right)=y^{-} d x^{-}+x^{+} d y^{+} .
$$

Получаем набор функций $S_{\kappa}$, порождающих симплектические отображения $F: D_{\kappa}^{-} \rightarrow D_{\kappa}^{+}$открытых множеств в $P$. Определим дискретный лагранжиан

$$
L_{\kappa}\left(z^{-}, z^{+}\right)=\left\langle x^{-}, y^{-}\right\rangle-S_{\kappa}\left(x^{-}, y^{+}\right), \quad z^{ \pm}=\left(x^{ \pm}, y^{ \pm}\right) .
$$

Тогда орбиты $\mathbf{z}=\left(z_{i}\right)$ отображения $F$ оказываются критическими точками дискретного функционала действия Пуанкаре

$$
\mathscr{A}_{\mathbf{k}}(\mathbf{z})=\sum L_{\kappa_{i}}\left(z_{i}, z_{i+1}\right)
$$

Таким образом, z - траектория ДЛС с $2 m$ степенями свободы. Это представление является стандартным в симплектической топологии (см., например, [59]). 
3.4. ДЛС, порожденная лагранжевым потоком. ДЛС естественным образом возникают в лагранжевых системах с непрерывным временем (НЛС). Рассмотрим НЛС с лагранжианом $L(q, \dot{q}, t)$ на $T M \times \mathbb{R}$, где $M$ - гладкое многообразие (конфигурационное пространство). Предполагается, что $L$ удовлетворяет условию Лежандра: преобразование Лежандра $\dot{q} \mapsto p=\partial_{\dot{q}} L-$ диффеоморфизм. Тогда $L$ определяет лагранжев поток на $T M$ или гамильтонов поток $\phi^{t}$ с гамильтонианом $H(q, p, t)=\langle p, \dot{q}\rangle-L$ на $T^{*} M$.

Если лагранжиан $T$-периодичен по времени, то динамика описывается отображением Пуанкаре (монодромии) $\phi^{T}: T^{*} M \rightarrow T^{*} M$. Это отображение симплектическое и может быть представлено с помощью ДЛС. В рассматриваемом случае это можно сделать более явно.

Определим функцию действия Гамильтона как действие траектории $\gamma$ : $[0, T] \rightarrow M$, соединяющей две точки $x_{-}, x_{+} \in M:$

$$
S\left(x_{-}, x_{+}\right)=\int_{0}^{T} L(\gamma(t), \dot{\gamma}(t), t) d t .
$$

Если крайние точки $x_{ \pm}$не сопряжены вдоль $\gamma$, то $S$ оказывается локально корректно определенной и гладкой. Согласно гамильтоновой формуле первой вариации, начальный и конечный импульсы $p_{-}=p(0)$ и $p_{+}=p(T)$ таковы, что

$$
p_{+} d x_{+}-p_{-} d x_{-}=d S\left(x_{-}, x_{+}\right) .
$$

Таким образом, $S$ - локальная производящая функция отображения монодромии $\left(q_{-}, p_{-}\right) \mapsto\left(q_{+}, p_{+}\right)$.

В общем случае может быть несколько траекторий, соединяющих $x_{-}$и $x_{+}$, так что функция $S$ многозначна. Таким образом, мы получаем набор производящих функций $S_{\kappa}$, определенных на открытых множествах $U_{\kappa}^{-} \times U_{\kappa}^{+} \subset M^{2}$. Пусть $\gamma: \mathbb{R} \rightarrow M$ - траектория НЛС и $x_{i}=\gamma\left(t_{i}\right), t_{i}=T i$. Если точки $x_{i}$ и $x_{i+1}$ не сопряжены вдоль $\gamma$, то $\mathbf{x}=\left(x_{i}\right)$ - траектория ДЛС $\mathscr{L}=\left\{S_{\kappa}\right\}$. Если выполнено условие Лежандра, то обратное также верно: траектория $\mathbf{x}$ ДЛС определяет траекторию $\gamma$ НЛС с импульсом, удовлетворяющим

$$
p\left(t_{i}+0\right)=-\partial_{x_{i}} L_{\kappa_{i}}\left(x_{i}, x_{i+1}\right)=\partial_{x_{i}} L_{\kappa_{i-1}}\left(x_{i-1}, x_{i}\right)=p\left(t_{i}-0\right) .
$$

Действительно, тогда импульс определяет скорость, поэтому из равенства $\Delta p\left(t_{i}\right)=0$ вытекает, что $\Delta \dot{\gamma}\left(t_{i}\right)=0$. Таким образом, $\gamma$ оказывается гладкой в $t_{i}$, а потому траекторией НЛС. Более того, дискретный функционал действия соответствует функционалу действия: $\int_{\gamma} L d t=A_{\mathbf{k}}(\mathbf{x})$.

ЗАмЕчАниЕ 2. Эту конструкцию можно обобщить. Возьмем гиперповерхности $\left\{\Sigma_{k}\right\}_{k \in I}$ в $M \times \mathbb{R}$. Для $\kappa=\left(\kappa_{-}, \kappa_{+}\right) \in I^{2}$ определим дискретный лагранжиан $L_{\kappa}\left(x_{-}, x_{+}\right), x_{ \pm}=\left(q_{ \pm}, t_{ \pm}\right) \in \Sigma_{\kappa_{ \pm}}$, как действие траектории $\gamma:\left[t_{-}, t_{+}\right] \rightarrow M$, соединяющей несопряженную пару точек $q_{-}, q_{+}$. В общей ситуации может быть несколько таких траекторий, так что мы получаем набор лагранжианов, определенных на открытых множествах в $\Sigma_{\kappa_{-}} \times \Sigma_{\kappa_{+}}$. Мы используем эту конструкцию в п. 6.3 . 
Для автономных систем любое $T>0$ является периодом и может быть использовано для определения отображения монодромии $\phi^{T}$. В этом случае $\phi^{T}$ имеет интеграл энергии $H$ и группу симметрий, определенную гамильтоновым потоком $\phi^{t}$. Соответствующая ДЛС будет иметь интеграл энергии и (локальную) группу симметрий. Поэтому такая ДЛС не может быть антиинтегрируемой.

Этой трудности можно избежать, заменив отображение монодромии $\phi^{T}$ отображением Пуанкаре. Зафиксируем энергию и рассмотрим локальное сечение $N$, трансверсальное потоку на уровне энергии $H=E$. Это можно сделать, выбрав гиперповерхность $\Sigma \subset M$ (в приложениях $\Sigma=\bigcup \Sigma_{k}$ может быть объединением нескольких гиперповерхностей) и положив

$$
N=\{(q, p): q \in \Sigma, H(q, p)=E\}
$$

Это многообразие симплектическое и отображение Пуанкаре $F: U \rightarrow N$ - симплектическое на открытом множестве $U \subset N$.

Представим $F$ с помощью ДЛС с $m-1$ степенями свободы следующим образом. Определим дискретный лагранжиан как действие Мопертюи

$$
S\left(x_{-}, x_{+}\right)=\int_{\gamma} p d q, \quad p=\partial_{\dot{q}} L,
$$

траектории $\gamma:[0, T] \rightarrow M$ энергии $H=E$, соединяющей данные точки $x_{ \pm} \in$ $M$. Здесь $T=T(\gamma)>0$ произвольно. Действие $S$ является гладким, если $x_{ \pm}$не сопряжены вдоль $\gamma$ для функционала действия Мопертюи. Действие удовлетворяет (3.14), но из уравнения Гамильтона-Якоби $H\left(x_{+}, \partial_{x_{+}} S\right)=E$ следует, что условие закручивания всегда нарушается.

Траектория $\gamma$ не всегда существует, и их может быть несколько, поэтому мы получаем набор дискретных лагранжианов $S_{\kappa}$ на открытых множествах $U_{\kappa}^{-} \times$ $U_{\kappa}^{+} \subset M^{2}$. Возьмем гиперповерхность $\Sigma \subset M$ и положим $\Sigma_{\kappa}^{ \pm}=\Sigma \cap U_{\kappa}^{ \pm}$. Тогда $L_{\kappa}=\left.S_{\kappa}\right|_{\Sigma_{\kappa}^{-} \times \Sigma_{\kappa}^{+}}-$локальная производящая функция отображения Пуанкаре сечения (3.15). Итак, $\mathscr{L}=\left\{L_{\kappa}\right\}$ - ДЛС, описывающая траектории непрерывной лагранжевой системы на уровне энергии $H=E$. Если $\gamma: \mathbb{R} \rightarrow M-$ траектория с $H=E$ такая, что $\gamma\left(t_{i}\right) \in \Sigma$ и точки $x_{i}, x_{i+1}$ не сопряжены (для фиксированной энергии) вдоль $\gamma$, то $\mathbf{x}=\left(x_{i}\right)$ - траектория ДЛС.

Отметим, что не все траектории ДЛС соответствуют траекториям непрерывной лагранжевой системы: некоторые из них соответствуют бильярдным траекториям с упругим отражением от $\Sigma$. Действительно, для данного $x \in \Sigma$ из уравнений

$$
H(x, p)=H\left(x, p^{\prime}\right), \quad \Delta p=p^{\prime}-p_{-} \perp T_{x} \Sigma
$$

не следует, что $p^{\prime}=p$. Если $H$ выпуклый по $p$, то имеется два решения для $p^{\prime}$ : одно описывает упругое отражение от $\Sigma$, а другое $\left(p^{\prime}=p\right)$ - гладкую траекторию.

Впрочем, в локальных задачах, обсуждаемых в этой статье, эта трудность не возникает: уравнения, написанные выше, имеют локально единственное решение. 


\section{4. Антиинтегрируемый предел в ДЛС}

4.1. Основная теорема. Исходная идея антиинтегрируемого предела [6] развивалась в нескольких направлениях. Случай многомерного стандартного отображения был рассмотрен в [57] и других работах - см. ссылки в [42]. Общий эквивариантный случай представлен в [70]. Бильярдные системы с малыми выпуклыми рассеивателями изучались в [30]. Частица малой массы в потенциальном силовом поле как непрерывная лагранжева система около антиинтегрируемого предела исследовалась в [18]. Другие примеры и ссылки можно найти в разделе 6 . В настоящем разделе мы укажем общий подход к антиинтегрируемому пределу в ДЛС, который обслуживает, в сущности, все известные примеры.

Рассмотрим ДЛС $\mathscr{L}=\left\{L_{\kappa}\right\}_{\kappa \in J}$, определенную в п. 3.2. В антиинтегрируемом пределе лагранжианы являются малыми возмущениями лагранжианов без закручивания $L_{\kappa}^{0}$ :

$$
L_{\kappa}(x, y)=L_{\kappa}^{0}(x, y)+u_{\kappa}(x, y), \quad \kappa \in J,
$$

где функция $u_{\kappa}$ мала в $C^{2}$-норме, а $L_{\kappa}^{0}$ имеет тождественно нулевое закручивание: $B_{\kappa}^{0}(x, y) \equiv 0$. Тогда

$$
L_{\kappa}^{0}(x, y)=V_{\kappa}^{-}(x)+V_{\kappa}^{+}(y),
$$

где функции $V_{\kappa}^{ \pm}$определены на множествах $U_{\kappa}^{ \pm}$соответственно.

Определим ориентированный граф Г с множеством вершин $J$ и множеством ребер $E$ следующим образом. Вершины $\kappa, \kappa^{\prime} \in J$ соединяются ребром $\gamma=$ $\left(\kappa, \kappa^{\prime}\right)$, если $W_{\gamma}=U_{\kappa}^{+} \cap U_{\kappa^{\prime}}^{-} \neq \varnothing$ и функция $\Psi_{\gamma}=V_{\kappa}^{+}+V_{\kappa^{\prime}}^{-}$на $W_{\gamma}$ имеет невырожденную критическую точку. Тогда каждому ребру $\gamma=\left(\kappa, \kappa^{\prime}\right) \in E$, соединяющему вершины $\kappa, \kappa^{\prime} \in J$, соответствует невырожденная критическая точка $a_{\gamma}$ функции $\Psi_{\gamma}$.

ЗАмЕчАниЕ 3 . Часто для данных $\kappa, \kappa^{\prime}$ критическая точка $a_{\gamma}$ будет единственной. Если имеется несколько невырожденных критических точек, можно получить более богатую символическую динамику, соединяя $\kappa, \kappa^{\prime}$ несколькими ребрами $\gamma$, каждому из которых соответствует своя невырожденная критическая точка $a_{\gamma}$. В этом случае $Г$ не является простым графом: две вершины могут быть соединены несколькими ребрами. Изложенная ниже конструкция работает, но путь в графе означает последовательность ребер, а не вершин.

Согласно традиционному определению путь в графе $Г$ - это последовательность вершин $\mathbf{k}=\left(\kappa_{i}\right)_{i \in \mathbb{Z}}$. Он определяет последовательность ребер $\left(\gamma_{i}\right)_{i \in \mathbb{Z}}$, где $\gamma_{i}$ соединяет вершину $\kappa_{i-1}$ с $\kappa_{i}$. Соответствующая последовательность критических точек

$$
\mathbf{a}(\mathbf{k})=\left(a_{\gamma_{i}}\right)_{i \in \mathbb{Z}}
$$

является траекторией лагранжевой системы без закручивания $\mathscr{L}^{0}=\left\{L_{\kappa}^{0}\right\}$, т. е. критической точкой функционала действия с разделенными переменными:

$$
A_{\mathbf{k}}^{0}(\mathbf{x})=\sum_{k \in \mathbb{Z}} \Psi_{\gamma_{i}}\left(x_{i}\right)
$$


Множество $\Pi \subset J^{\mathbb{Z}}$ всех путей инвариантно относительно сдвига $\mathscr{T}: \Pi \rightarrow \Pi$, $\left(\kappa_{i}\right) \mapsto\left(\kappa_{i+1}\right)$. Предположим для простоты, что граф Г конечен. Общий случай обсуждается ниже в п. 4.3. При этом предположении множество П всех путей является компактным инвариантным относительно сдвига подмножеством в канторовом множестве $J^{\mathbb{Z}}$. Динамическая система $\mathscr{T}: \Pi \rightarrow \Pi$ называется топологической марковской цепью или подсдвигом конечного типа [52]. Если граф полный, т. е. $\Pi=J^{\mathbb{Z}}$, то имеем сдвиг Бернулли на пространстве из \#J символов.

Теорема 5. Предположим, ито \#Г $<\infty$. Тогда существуют $C, \varepsilon_{0}, \sigma>0$ такие, что если

$$
\varepsilon=\max _{\kappa}\left\|u_{\kappa}\right\|_{C^{2}}<\varepsilon_{0}
$$

то верно следующее.

(а) Для любого пути $\mathbf{k}=\left(\kappa_{i}\right)$ в Г существует единственная орбита $\mathbf{x}(\mathbf{k})=$ $\left(x_{i}\right)$ в ДЛС $\mathscr{L}$, которая отслеживает последовательность $\mathbf{a}=\mathbf{a}(\mathbf{k})$ :

$$
\rho(\mathbf{x}, \mathbf{a})=\sup _{i} \operatorname{dist}\left(x_{i}, a_{\gamma_{i}}\right)<\sigma
$$

Более того, $\rho(\mathbf{x}, \mathbf{a})<C \varepsilon$.

(b) Орбита $\mathbf{x}$ гиперболична с показателем Ляпунова $\lambda \geqslant C|\ln \varepsilon|$.

(c) Отображение $\mathbf{k} \mapsto \mathbf{x}$ непрерывно, $\operatorname{ma\kappa }$ что $\Lambda=\{(\mathbf{k}, \mathbf{x}(\mathbf{k})): \mathbf{k} \in \Pi\}-$ компактное множество в $J^{\mathbb{Z}} \times\left(\mathbb{R}^{m}\right)^{\mathbb{Z}}$.

ЗАмЕчаниЕ 4. Предположение, что $\Psi_{\gamma}$ имеет невырожденную критическую точку, можно ослабить. Например, для существования отслеживающей траектории достаточно, чтобы $\Psi_{\gamma}$ имело компактное множество точек минимума в $W_{\gamma}$. Но тогда отслеживающая орбита может не быть единственной или гиперболичной.

Если граф Г ветвится, т. е. существуют 2 цикла, проходящие через одну и ту же вершину, то $\mathscr{T}: \Lambda \rightarrow \Lambda$ имеет положительную топологическую энтропию. Если ДЛС допускает объемлющую систему $F: P \rightarrow P$, получаем гомеоморфизм $\Pi \rightarrow \Lambda \rightarrow \mathscr{K}$ на компактное гиперболическое множество $\mathscr{K} \subset P$ и $F: \mathscr{K} \rightarrow \mathscr{K}$ оказывается сопряженным топологической марковской цепи $\mathscr{T}: \Pi \rightarrow \Pi$. В этом случае справедливо следующее утверждение.

СлЕДСтвиЕ 1. Если граф Г ветвится, то множество $\mathscr{K}$ имеет положительную топологическую энтропию, удовлетворяющую (2.18).

ЗАмЕчАниЕ 5. Для наших целей требуется лишь локальное существование объемлющей системы в окрестности гиперболического множества. Таким образом, нужно лишь локальное условие закручивания:

$$
\operatorname{det} B_{\kappa}\left(a_{\gamma}, a_{\gamma^{\prime}}\right) \neq 0
$$

для любых двух ребер таких, что $\gamma$ заканчивается в $\kappa$, а $\gamma^{\prime}$ начинается в $\kappa$. 
В теореме 5 не требуется условия закручивания. Поэтому нам придется обобщить традиционное определение гиперболичности. В самом деле, без условия закручивания текущее состояние траектории не определяет прошлое и будущее, так что обычное определение устойчивого (сжимающего) и неустойчивого (растягивающего) подпространств не работает. Мы переформулируем конический критерий гиперболичности В. М. Алексеева [52].

Линеаризация уравнения (3.10) в х дает уравнение в вариациях траектории $(\mathbf{k}, \mathbf{x})$ :

$$
G_{i-} u_{i-1}+G_{i} u_{i}+G_{i+} u_{i+1}=0,
$$

где $G_{i}$ и $G_{i \pm}$ - линейные операторы. Определим конусы

$$
\begin{aligned}
H_{i} & =\left\{\left(u_{i}, u_{i+1}\right):\left\|u_{i}\right\| \leqslant \alpha_{H}\left\|u_{i+1}\right\|\right\}, \\
V_{i} & =\left\{\left(u_{i}, u_{i+1}\right):\left\|u_{i+1}\right\| \leqslant \alpha_{V}\left\|u_{i}\right\|\right\} .
\end{aligned}
$$

Будем говорить, что траектория $(\mathbf{k}, \mathbf{x})$ гиперболична, если существует $\mu>1$ такое, что для любого $i \in \mathbb{Z}$ и любых $u_{i-1}, u_{i}, u_{i+1}$, удовлетворяющих (4.3),

$$
\begin{aligned}
& \left(u_{i-1}, u_{i}\right) \in H_{i-1} \quad \text { влечет } \quad\left(u_{i}, u_{i+1}\right) \in H_{i} \text { и }\left\|\left(u_{i}, u_{i+1}\right)\right\| \geqslant \mu\left\|\left(u_{i-1}, u_{i}\right)\right\|, \\
& \left(u_{i}, u_{i+1}\right) \in V_{i} \quad \text { влечет } \quad\left(u_{i-1}, u_{i}\right) \in V_{i-1} \text { и }\left\|\left(u_{i-1}, u_{i}\right)\right\| \geqslant \mu\left\|\left(u_{i}, u_{i+1}\right)\right\| .
\end{aligned}
$$

Коническое определение имеет такой вид только при удачном выборе метрики. Но для нас это не важно, поскольку мы используем его только в качестве достаточного условия гиперболичности.

Если $\left(u_{i}, u_{i+1}\right) \in H_{i}$, то $\left\|\left(u_{j}, u_{j+1}\right)\right\| \geqslant \mu^{j-i}\left\|\left(u_{i}, u_{i+1}\right)\right\|$ при $j \geqslant i$, так что показатель Ляпунова не меньше $\log \mu$.

Будем говорить, что компактное $\mathscr{T}$-инвариантное множество $\Lambda$ траекторий $(\mathbf{k}, \mathbf{x})$ гиперболично, если каждая траектория $(\mathbf{k}, \mathbf{x})$ из $\Lambda$ удовлетворяет приведенным выше условиям с одними и теми же постоянными. Если ДЛС допускает объемлющую систему, то соответствующее компактное инвариантное множество $\mathscr{K} \subset P$ гиперболично в традиционном [52] смысле.

ЗАмечАниЕ 6. Другое условие гиперболичности для $(\mathbf{k}, \mathbf{x})$ состоит в том, что оператор Гессе $A_{\mathbf{k}}^{\prime \prime}(\mathbf{x}): l_{\infty}\left(\mathbb{R}^{m}\right) \rightarrow l_{\infty}\left(\mathbb{R}^{m}\right)$, определенный правой частью уравнения в вариациях, имеет ограниченный обратный в $l_{\infty}$-норме. Эквивалентность этих определений доказана в [7].

4.2. Доказательство теоремы 5. Доказательство теоремы почти такое же, как и в случае стандартного отображения. Согласно теореме о неявной функции для любого ребра $\gamma=\left(\kappa, \kappa^{\prime}\right) \in E$, соединяющего $\kappa, \kappa^{\prime} \in J$, отображение $g_{\gamma}=D \Psi_{\gamma}-$ диффеоморфизм из окрестности $W_{\gamma}$ точки $a_{\gamma}$ на окрестность начала координат в $\mathbb{R}^{m}$. Можно считать, что

$$
W_{\gamma}=\left\{x \in \mathbb{R}^{m}:\left|x-a_{\gamma}\right|<r\right\} .
$$

Здесь $r>0$ можно взять не зависящим от $\gamma$, поскольку граф Г конечен. Пусть $\phi_{\gamma}$ обозначает обратное отображение: $\phi_{\gamma}=g_{\gamma}^{-1}$. Тогда $\phi_{\gamma}(0)=a_{\gamma}$. 
Пусть $\mathbf{k}=\left(\kappa_{i}\right)$ - путь в графе Г и $\left(\gamma_{i}\right)$ - соответствующая последовательность ребер. Рассмотрим метрическое пространство $(X, \rho)$, где $X=\prod_{i \in \mathbb{Z}} W_{\gamma_{i}}$ и для
любых $\mathbf{x}, \mathbf{x}^{\prime} \in X$

$$
\rho\left(\mathbf{x}, \mathbf{x}^{\prime}\right)=\sup _{i \in \mathbb{Z}}\left|x_{i}-x_{i}^{\prime}\right| .
$$

Траектория $\mathbf{x}$ является критической точкой функционала $A_{\mathbf{k}}$ в окрестности точки $\mathbf{a}=\mathbf{a}(\mathbf{k})$ в $X$ тогда и только тогда, когда

$$
x_{i}=\phi_{\gamma_{i}}\left(\partial_{x_{i}}\left(u_{\kappa_{i-1}}\left(x_{i-1}, x_{i}\right)+u_{\kappa_{i}}\left(x_{i}, x_{i+1}\right)\right)\right) .
$$

Остается применить принцип сжимающих отображений в окрестности точки а в $X$. Пусть $\mathscr{B} \subset X-$ шар

$$
\mathscr{B}=\{\mathbf{x} \in X: \rho(\mathbf{x}, \mathbf{a}) \leqslant \sigma\}, \quad \sigma<r .
$$

Рассмотрим оператор $\Phi: \mathscr{B}_{\sigma} \rightarrow X$, действующий по правилу

$$
\mathbf{x} \mapsto \mathbf{y}=\Phi(\mathbf{x}), \quad y_{i}=\phi_{\gamma_{i}}\left(\partial_{x_{i}}\left(u_{\kappa_{i-1}}\left(x_{i-1}, x_{i}\right)+u_{\kappa_{i}}\left(x_{i}, x_{i+1}\right)\right)\right) .
$$

Любая неподвижная точка $\Phi$ является траекторией ДЛС $\mathscr{L}$.

Как и в разделе 2 , легко показать, что если $\varepsilon$ достаточно мало, то $\Phi\left(\mathscr{B}_{\sigma}\right) \subset$ $\mathscr{B}_{\sigma}$ и оператор $\Phi$ является сжимающим. Действительно,

$$
\begin{aligned}
\rho(\mathbf{y}, \mathbf{a}) & =\sup _{i \in \mathbb{Z}}\left|\phi_{\gamma_{i}}\left(\partial_{x_{i}}\left(u_{\kappa_{i-1}}\left(x_{i-1}, x_{i}\right)+u_{\kappa_{i}}\left(x_{i}, x_{i+1}\right)\right)\right)-\phi_{\gamma_{i}}(0)\right| \\
& \leqslant \lambda \sup _{i \in \mathbb{Z}}\left|\partial_{x_{i}}\left(u_{\kappa_{i-1}}\left(x_{i-1}, x_{i}\right)+u_{\kappa_{i}}\left(x_{i}, x_{i+1}\right)\right)\right| \leqslant 2 \lambda \varepsilon .
\end{aligned}
$$

Поэтому $\mathbf{y} \in \mathscr{B}_{\sigma}$, если взять $\sigma=2 \lambda \varepsilon$.

Положим $\mathbf{y}=\Phi(\mathbf{x}), \mathbf{y}^{\prime}=\Phi\left(\mathbf{x}^{\prime}\right), \mathbf{x}, \mathbf{x}^{\prime} \in \mathscr{B}_{\sigma}$. Тогда

$$
\begin{aligned}
\rho\left(\mathbf{y}, \mathbf{y}^{\prime}\right)=\sup _{i \in \mathbb{Z}} \mid \phi_{\gamma_{i}}\left(\partial_{x_{i}}\left(u_{\kappa_{i-1}}\left(x_{i-1}, x_{i}\right)+u_{\kappa_{i}}\left(x_{i}, x_{i+1}\right)\right)\right) \\
\quad-\phi_{\gamma_{i}}\left(\partial_{x_{i}^{\prime}}\left(u_{\kappa_{i-1}}\left(x_{i-1}^{\prime}, x_{i}^{\prime}\right)+u_{\kappa_{i}}\left(x_{i}^{\prime}, x_{i+1}^{\prime}\right)\right)\right) \mid \\
\leqslant \sup _{i \in \mathbb{Z}} \mid \partial_{x_{i}}\left(u_{\kappa_{i-1}}\left(x_{i-1}, x_{i}\right)+u_{\kappa_{i}}\left(x_{i}, x_{i+1}\right)\right) \\
\quad-\partial_{x_{i}^{\prime}}\left(u_{\kappa_{i-1}}\left(x_{i-1}^{\prime}, x_{i}^{\prime}\right)+u_{\kappa_{i}}\left(x_{i}^{\prime}, x_{i+1}^{\prime}\right)\right) \mid \\
\leqslant 2 \lambda \varepsilon \rho\left(\mathbf{x}, \mathbf{x}^{\prime}\right) .
\end{aligned}
$$

Поэтому $\Phi$ является сжимающим при $\varepsilon<1 /(2 \lambda)$.

Теперь докажем утверждение (b). B (4.4), (4.5) возьмем $\alpha_{H}=\alpha_{V}=1 / 2$. Пусть $\mathbf{x}$ - орбита, соответствующая $\mathbf{k} \in$ П согласно утверждению (а). Дифференцируя (4.9), получаем

$$
u_{i}=P_{i} u_{i-1}+Q_{i} u_{i}+R_{i} u_{i-1} .
$$

Это равенство эквивалентно (4.3).

Нормы линейных операторов $P_{i}, Q_{i}, R_{i}$ малы:

$$
\left\|P_{i}\right\| \leqslant \lambda \varepsilon, \quad\left\|Q_{i}\right\| \leqslant \lambda \varepsilon, \quad\left\|R_{i}\right\| \leqslant \lambda \varepsilon .
$$


Из равенств

$$
R_{i} u_{i+1}=\left(I-Q_{i}\right) u_{i}-P_{i} u_{i-1}, \quad\left(u_{i-1}, u_{i}\right) \in H_{i-1},
$$

следует, что

$$
\frac{\left|u_{i}\right|}{\left|u_{i+1}\right|} \leqslant \frac{\left|\left(I-Q_{i}\right) u_{i}-P_{i} u_{i-1}\right|}{\left(1-\left\|Q_{i}\right\|-\left\|P_{i}\right\| / 2\right)\left|u_{i+1}\right|} \leqslant \frac{\left|R_{i} u_{i+1}\right|}{(1-3 \varepsilon \lambda / 2)\left|u_{i+1}\right|} \leqslant \frac{\varepsilon \lambda}{1-3 \varepsilon \lambda / 2} .
$$

Таким образом,

$$
\left(u_{i}, u_{i+1}\right) \in H_{i} \quad \text { при условии } \frac{\varepsilon \lambda}{1-3 \varepsilon \lambda / 2}<\frac{1}{2} .
$$

Отсюда следует первое утверждение в (4.6). Имеем также:

$$
\begin{aligned}
\left\|\left(u_{i-1}, u_{i}\right)\right\|^{2} & \leqslant\left|u_{i-1}\right|^{2}+\frac{\left|\left(I-Q_{i}\right) u_{i}-P_{i} u_{i-1}\right|^{2}}{(1-\|Q\|-\|P\| / 2)^{2}} \\
& \leqslant \frac{\left|u_{i}\right|^{2}}{4}+\frac{\varepsilon^{2} \lambda^{2}\left|u_{i+1}\right|^{2}}{(1-3 \varepsilon \lambda / 2)^{2}} \leqslant \frac{1}{4}\left\|\left(u_{i}, u_{i+1}\right)\right\|^{2}
\end{aligned}
$$

при условии $\varepsilon \lambda /(1-3 \varepsilon \lambda)<1 / 2$. Следовательно, получаем второе утверждение в (4.6) с $\mu=2$. Утверждения (4.7) проверяются аналогично.

Докажем (c). Если два кода $\mathbf{k}$ и $\mathbf{k}^{\prime}$ близки в топологии прямого произведения в $J^{\mathbb{Z}}$, то они имеют длинный одинаковый отрезок: $\kappa_{i}=\kappa_{i}^{\prime}$ при $|i| \leqslant n$. Тогда соответствующие траектории $\mathbf{x}$ и $\mathbf{x}^{\prime}$ таковы, что

$$
\left|x_{i}-x_{i}^{\prime}\right| \leqslant C \alpha^{|i|-n}, \quad 0<\alpha<1, \quad|i| \leqslant n .
$$

Это следует из (4.9), как и в доказательстве леммы 2. Если $n$ велико, то х близка к $\mathrm{x}^{\prime}$ в топологии прямого произведения. Следовательно, отображение $\mathbf{k} \mapsto \mathbf{x}$ непрерывно. Теорема 5 доказана.

Другой способ проверки гиперболичности состоит в том, чтобы проверить, что гессиан $A_{\mathbf{k}}^{\prime \prime}(\mathbf{x})$ имеет ограниченный обратный в $l^{\infty}\left(\mathbb{R}^{m}\right)$-норме. Так как он трехдиагонален с обратимой диагональю и малыми недиагональными членами, это почти очевидно. Можно также описать устойчивое и неустойчивое подпространства гиперболической траектории.

ПреДЛОжеНИЕ 1. Для любого $u \in \mathbb{R}^{m}$ существует единственная траектория $\mathbf{u}=\left(u_{j}\right)_{j \geqslant 0}$ вариационной системы такая, что $u_{0}=u u\left\|u_{j}\right\|$ ограничен при $j \rightarrow \infty$. Траектория и экспоненциально стремится к нулю: $\left\|u_{j}\right\| \leqslant$ $\mu^{-j}\|u\|, \mu>1$. Таким образом, $\mathbf{u}$ лежит в устойчивом подпространстве траектории $(\mathbf{k}, \mathbf{x})$. Случай неустойчивого подпространства аналогичен.

Доказательство предложения 1 получается с помощью тех же рассуждений в терминах принципа сжатия, примененных к отображению

$$
\left(u_{i}\right)_{i>0} \mapsto\left(v_{i}\right)_{i>0}, \quad v_{i}=P_{i} u_{i-1}+Q_{i} u_{i}+R_{i} u_{i-1}, \quad u_{0}=u
$$

(см. (4.10)) на пространстве ограниченных последовательностей $\left(u_{j}\right)_{j>0}$. 
4.3. G-эквивариантные ДЛС. Во многих приложениях антиинтегрируемый предел используется для построения траекторий, уходящих на бесконечность. Тогда приходится рассматривать бесконечные графы Г. В случае бесконечного графа Г требуются предположения о равномерности.

УСловие $\mathrm{U}$ (равномерная антиинтегрируемость). Существуют положительные постоянные $r$ и $\lambda$ такие, что для любого $\gamma \in E$

(а) $g_{\gamma}: W_{\gamma} \rightarrow B_{\gamma} \subset \mathbb{R}^{m}$ - диффеоморфизм, где $B_{\gamma}$ - окрестность нуля и $W_{\gamma}$ определено в (4.8) с $r>0$, не зависящим от $\gamma$;

(b) отображение $\phi_{\gamma}=g_{\gamma}^{-1}: B_{\gamma} \rightarrow W_{\gamma}$ липшицево с постоянной Липшица $\lambda$;

(c) $\varepsilon=\sup _{\kappa \in J}\left\|u_{\kappa}\right\|_{C^{2}}$ конечно и достаточно мало.

Очевидно, что условие U выполнено, если Г конечен.

ТеОрема 6. Теорема 5 остается верной для бесконечного графа Г, если ДЛС равномерно антиинтегрируема.

ДокАЗАТЕльство совпадает с доказательством теоремы 5.

Условие U весьма ограничительно. В этом пункте мы укажем класс ДЛС с $\# E=\infty$, в котором условие $U$ требует выполнения лишь конечного числа условий.

Предположим, что на $M$ действует дискретная группа ${ }^{5} G$. Предположим, что действие дискретно: любая точка $x \in M$ имеет окрестность $U$ такую, что множества $g(U), g \in G$, не пересекаются: $g^{\prime}(U) \cap g^{\prime \prime}(U)=\varnothing$, если $g^{\prime} \neq g^{\prime \prime}$. В этом случае факторпространство $\widetilde{M}=M / G$ - гладкое многообразие и $\pi: M \rightarrow \widetilde{M}-$ накрытие. Действие $G$ на $M$ порождает диагональное действие $G$ на произведении $M \times M$ : для любой пары $x, y \in M$ и $g \in G$ имеем $g(x, y)=(g(x), g(y))$.

Пусть $L: M \times M \rightarrow \mathbb{R}$ инвариантен относительно действия $G$ :

$$
L(x, y)=L(g(x, y)) \quad \text { при всех } x, y \in M \text { и } g \in G \text {. }
$$

Соответствующая ДЛС называется $G$-эквивариантной. Объемлющую систему в этом случае можно определить как отображение $F: P \rightarrow P, P=(M \times M) / G$. В случае многомерного стандартного отображения с лагранжианом (3.6) имеем $M=\mathbb{R}^{m}$ и $G \cong \mathbb{Z}^{m}$ - группа сдвигов, сохраняющих потенциал $V$.

Рассмотрим другой пример. Предположим, что $M$ - риманово многообразие и группа $G$ действует на $M$ изометриями. Пусть $\operatorname{dist}(\cdot, \cdot)-\operatorname{paccтояние,~}$ индуцированное римановой метрикой. Тогда лагранжиан $L(x, y)=\operatorname{dist}^{2}(x, y)-$ гладкая функция для любой пары достаточно близких точек $x, y$. Он инвариантен относительно диагонального действия $G$.

Для гладких $G$-инвариантных функций $V: M \rightarrow \mathbb{R}$ и $u: M \times M \rightarrow \mathbb{R}$ рассмотрим ДЛС, заданную дискретным лагранжианом

$$
L(x, y)=u(x, y)+V(y) .
$$

Определим антиинтегрируемый предел в такой системе как предел $\|u\|_{C^{2}} \rightarrow 0$.

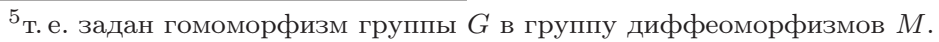


Если динамика в системе с этим лагранжианом глобально определена, можно считать определенной объемлющую систему.

Предположим, что конфигурационное пространство $\widetilde{M}=M / G$ компактно. Зафиксируем риманову метрику на $\widetilde{M}$, которая поднимается до $G$-инвариантной метрики на $M$. Пусть dist - соответствующее расстояние на $M$.

Пусть $\mathrm{Cr} \subset \widetilde{M}$ - конечное множество невырожденных критических точек функции $V$ на $\widetilde{M}$ и $I$ - соответствующее $G$-инвариантное множество критических точек $V$ на $M$. Последнее является конечным объединением орбит

$$
\{g(k): g \in G\}, \quad k \in I,
$$

действия группы. Возьмем малую $\rho$-окрестность множества $\operatorname{Cr}$ в $\widetilde{M}$, соответствующую $G$-инвариантной окрестности $U=\bigcup_{k \in I} U_{k}$ множества $I$ в $M$.

Для большой постоянной $N$ определим граф $\Gamma$ :

$J=\left\{\kappa=\left(\kappa_{-}, \kappa_{+}\right) \in I^{2}: \operatorname{dist}\left(\kappa_{-}, \kappa_{+}\right)<N\right\}, \quad E=\left\{\gamma=\left(\kappa, \kappa^{\prime}\right) \in J^{2}: \kappa_{+}=\kappa_{-}^{\prime}\right\}$.

В обозначениях п. 4.1

$$
L_{\kappa}=\left.L\right|_{U_{\kappa_{-}} \times U_{\kappa_{+}}}, \quad V_{\kappa}^{-}=0, \quad V_{\kappa}^{+}=\left.V\right|_{U_{\kappa_{+}}}, \quad u_{\kappa}=\left.u\right|_{U_{\kappa_{-}} \times U_{\kappa_{+}}} .
$$

Если группа $G$ бесконечна, то граф Г бесконечен, но в силу $G$-инвариантности $L$ условие $\mathrm{U}$ следует из конечности множества $\mathrm{Cr}$ и ограниченности $N$. Отметим, что $G$ действует также на $\Gamma$ и $\widetilde{\Gamma}=\Gamma / G-$ конечный граф. Таким образом, получаем следующее утверждение.

ПредлОЖениЕ 2. Отображение $F$ имеет гиперболическое множество $K$ такое, что $\left.F\right|_{K}$ сопряжено топологической марковской цепи, определенной графом $\Gamma$.

На самом деле требуется лишь локальное существование объемлющей системы около гиперболического множества. Она существует, если $\operatorname{det} B\left(\kappa_{-}, \kappa_{+}\right) \neq 0$ при всех $\left(\kappa_{-}, \kappa_{+}\right) \in J$.

Отметим, что если $M$ некомпактно, то $M^{2} / G$ - также некомпактно, но множество $K$ компактно, поскольку все его точки расположены на расстоянии меньшем, чем $N+\sigma$, от компактного множества $\left\{(x, x) \in M^{2}\right\} / G$.

\section{5. Примеры: системы с дискретным временем}

5.1. Легкая частица и периодические толчки. Рассмотрим частицу малой массы $\varepsilon^{2}$, движущуюся в пространстве $\mathbb{R}^{m}$ в поле сил с потенциалом

$$
\mathscr{V}(x, t)=\frac{1}{2 \pi} V(x) \delta(t),
$$

где $V$ - гладкая функция на $\mathbb{R}^{m}$, а $\delta$ - периодическая $\delta$-функция:

$$
\begin{gathered}
\delta(t)= \begin{cases}\infty, & t \in 2 \pi \mathbb{Z}, \\
0, & t \in \mathbb{R} \backslash 2 \pi \mathbb{Z},\end{cases} \\
\int_{2 \pi k-\sigma}^{2 \pi k+\sigma} \delta(t) d t=1 \quad \text { для любых } k \in \mathbb{Z}, \sigma \in(0, \pi) .
\end{gathered}
$$


Гамильтониан системы имеет вид

$$
H=\frac{1}{2 \varepsilon^{2}}|p|^{2}+\frac{1}{2 \pi} V(x) \delta(t),
$$

где $p=\left(p_{1}, \ldots, p_{m}\right)$ - импульс, канонически сопряженный координатам $x=$ $\left(x_{1}, \ldots, x_{m}\right)$. Уравнения Гамильтона - следующие:

$$
\dot{p}=-\frac{1}{2 \pi} \frac{\partial V}{\partial x}(x) \delta(t), \quad \dot{x}=\frac{p}{\varepsilon^{2}} .
$$

Таким образом, $p$ получает приращения $-(2 \pi)^{-1} \partial V / \partial x$ в моменты времени $2 \pi l$, $l \in \mathbb{Z}$. В течение остального времени частица движется свободно.

Для любого целого $l$ положим $x(2 \pi l-0)=x_{l}, p(2 \pi l-0)=p_{l}$. Тогда

$$
\begin{gathered}
\left(\begin{array}{l}
x_{l} \\
p_{l}
\end{array}\right) \mapsto\left(\begin{array}{c}
x(2 \pi l+0) \\
p(2 \pi l+0)
\end{array}\right)=\left(\begin{array}{c}
x_{l} \\
p_{l}-\frac{1}{2 \pi} \frac{\partial V}{\partial x}\left(x_{l}\right)
\end{array}\right) \\
\mapsto\left(\begin{array}{l}
x_{l+1} \\
p_{l+1}
\end{array}\right)=\left(\begin{array}{c}
x_{l}+2 \pi \varepsilon^{-2} p_{l+1} \\
p_{l}-\frac{1}{2 \pi} \frac{\partial V}{\partial x}\left(x_{l}\right)
\end{array}\right) .
\end{gathered}
$$

Величины $x_{l-1}, x_{l}, x_{l+1}$ удовлетворяют уравнению

$$
x_{l+1}-2 x_{l}+x_{l-1}=\varepsilon^{-2} \frac{\partial V}{\partial x}\left(x_{l}\right) .
$$

Имеем ДЛС с лагранжианом вида (3.6), где $B=\varepsilon^{2} I$ :

$$
L(x, y)=\varepsilon^{2} \frac{|x-y|^{2}}{2}-V(y) .
$$

Соответствующая дискретная динамическая система - многомерное стандартное отображение. Согласно теореме 5 , если $V$ имеет две невырожденные критические точки, то при малых $\varepsilon$ система имеет хаотическое гиперболическое множество. Если $V$ является $\mathbb{Z}$-периодическим на $\mathbb{R}^{m}$, то имеем $\mathbb{Z}$-эквивариантную ДЛС, соответствующую симплектическому закручивающему отображению $\mathbb{T}^{m} \times \mathbb{R}^{m}$ на себя.

Отметим, что при малых $\varepsilon$ система остается близкой к антиинтегрируемому пределу, если потенциал $V$ - гладкая периодическая функция, близкая (как обобщенная функция) к (5.1).

Если легкая частица движется в потенциальном силовом поле, причем потенциал $V(x, t)$ не удовлетворяет (5.1), то теория антиинтегрируемого предела становится технически более трудной. Мы обсуждаем эти результаты в п. 6.3.

5.2. Бильярд в широкой полосе. Рассмотрим плоский бильярд в широкой полосе, ограниченной графиками двух 1-периодических функций. Другими словами, рассмотрим частицу, движущуюся в области

$$
D=\left\{(x, y) \in \mathbb{R}^{2}: f_{1}(x) \leqslant y \leqslant f_{2}(x)+d\right\},
$$




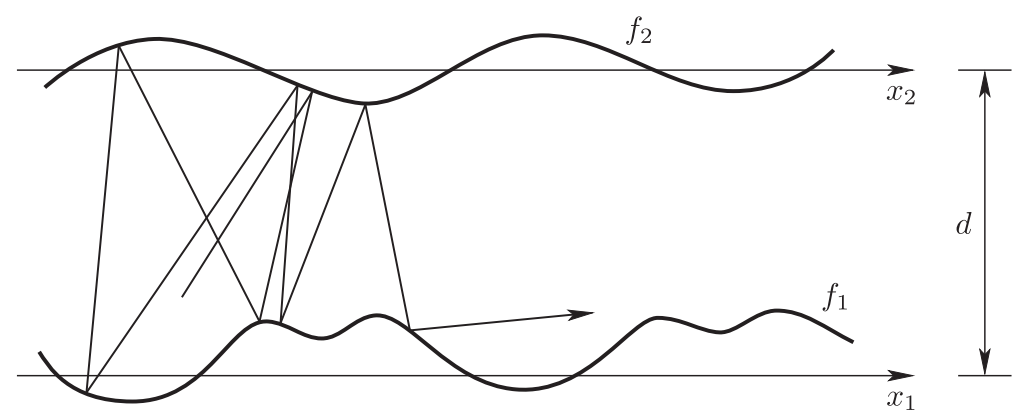

Рис. 1. Бильярд в широкой полосе

где $f_{1}, f_{2}-1$-периодические функции, а параметр $d$ большой (рис. 1 ). Движение частицы внутри области предполагается свободным, а отражения от границы упругими.

Это бильярдная система (см. п. 3.1). Пусть $L$ - длина отрезка между двумя последовательными точками удара о границу. Мы будем рассматривать движения такие, что частица ударяется поочередно о нижнюю и верхнюю стенки.

Пусть $x_{1}$ - координата на нижней границе, а $x_{2}$ - на верхней. Длина соответствующего отрезка выражается формулой

$$
L\left(x_{1}, x_{2}\right)=\sqrt{\left(x_{2}-x_{1}\right)^{2}+\left(d+f_{2}\left(x_{2}\right)-f_{1}\left(x_{1}\right)\right)^{2}} .
$$

Лагранжиан $\mathbb{Z}$-инвариантен и при больших $d$ имеет вид (3.6):

$$
L\left(x_{1}, x_{2}\right)=d+f_{2}\left(x_{2}\right)-f_{1}\left(x_{1}\right)+\frac{\varepsilon}{2}\left(x_{2}-x_{1}\right)^{2}+O\left(\varepsilon^{2}\right), \quad d=\varepsilon^{-1} .
$$

Функционал действия - следующий:

$$
A(\mathbf{x})=\sum_{i \in \mathbb{Z}}\left(L\left(x_{2 i-1}, x_{2 i}\right)+L\left(x_{2 i+1}, x_{2 i}\right)\right) .
$$

В обозначениях п. 3.2 имеем ДЛС $\mathscr{L}=\left\{L_{1}, L_{2}\right\}$ с конфигурационным пространством $M=\mathbb{R}_{1} \cup \mathbb{R}_{2}$ (являющимся объединением двух копий $\mathbb{R}$ ) и лагранжианом $L_{1}\left(x_{1}, x_{2}\right)=L\left(x_{1}, x_{2}\right)$ и $L_{2}\left(x_{2}, x_{1}\right)=L\left(x_{1}, x_{2}\right)$. В функционале действия

$$
A(\mathbf{x})=\sum_{i \in \mathbb{Z}} L_{\kappa_{i}}\left(x_{i}, x_{i+1}\right)=\sum_{i \in \mathbb{Z}}\left(d+2(-1)^{i} f_{\kappa_{i}}\left(x_{i}\right)+O(\varepsilon)\right), \quad \kappa_{i}=i \bmod 2,
$$

переменные разделены (см. (4.1)). Таким образом, ДЛС антиинтегрируема, если функции $f_{1}$ и $f_{2}$ имеют невырожденные критические точки. Пусть $\mathrm{Cr}_{1} \subset \mathbb{R}_{1}$ и $\mathrm{Cr}_{2} \subset \mathbb{R}_{2}$ обозначают $\mathbb{Z}$-инвариантные множества невырожденных критических точек функций $f_{1}$ и $f_{2}$. Предположим, что множества $\mathrm{Cr}_{1} / \mathbb{Z}$ и $\mathrm{Cr}_{2} / \mathbb{Z}$ конечны и непусты. 
Соответствующий граф Г имеет вершины двух типов: $J=J_{1} \cup J_{2}$, где

$$
\begin{aligned}
& J_{1}=\left\{(\kappa, x, y) \in\{1\} \times \mathrm{Cr}_{1} \times \mathrm{Cr}_{2}:|x-y|<N\right\}, \\
& J_{2}=\left\{(\kappa, x, y) \in\{2\} \times \mathrm{Cr}_{2} \times \mathrm{Cr}_{1}:|x-y|<N\right\} .
\end{aligned}
$$

Вершины $(\kappa, x, y)$ и $\left(\kappa^{\prime}, x^{\prime}, y^{\prime}\right)$ соединены ребром тогда и только тогда, когда $\kappa \neq \kappa^{\prime}$ и $y=x^{\prime}$.

Как и в стандартной эквивариантной ситуации (п. 4.3), получаем существование гиперболического множества, несущего динамику, сопряженную динамике в топологической марковской цепи. Граф Г бесконечен, но условие равномерной антиинтегрируемости выполняется.

Многомерный аналог этой системы получается непосредственно: достаточно сказать, что $x \in \mathbb{R}^{m}$ и функции $f_{1}, f_{2}$ периодичны относительно всех компонент векторной переменной $x$.

5.3. Бильярды с малыми рассеивателями. Пусть $D \subset \mathbb{R}^{m}$ - область с гладкой границей $\Sigma=\partial D$. Следуя [30], рассмотрим бильярд в области $\Omega_{\varepsilon}=$ $D \backslash\left(\bigcup_{j=1}^{N} D_{j}\right)$, где $D_{j} \subset D$ - малые подобласти, играющие роль рассеивателей. Они малы в следующем смысле. Каждая область $D_{j}$ ассоциируется с некоторой точкой $a_{j} \in D$. Граница $\partial D_{j}$ имеет вид

$$
a_{j}+\varepsilon \Sigma_{j}=\left\{q \in \mathbb{R}^{m}: q=a_{j}+\varepsilon \phi_{j}(x), x \in S^{m-1}\right\},
$$

где вектор-функции $\phi_{j}: S^{m-1} \rightarrow \mathbb{R}^{m}$ являются гладкими вложениями, которые определяют гладкие подмногообразия $\Sigma_{j}=\phi_{j}\left(S^{m-1}\right)$. Если $\varepsilon$ достаточно мало, то $D_{j} \subset D$. Когда $\varepsilon \rightarrow 0$, область $\Omega_{\varepsilon}$ вырождается в $\Omega_{0}=D \backslash A$, где $A=$ $\left\{a_{1}, \ldots, a_{N}\right\}$.

Мы собираемся объяснить, что при малых $\varepsilon$ этот бильярд порождает ДЛС с дискретным лагранжианом $\mathscr{L}=\left\{L_{\kappa}\right\}$, где индекс $\kappa$ соответствует невырожденной траектории бильярда в $D$, начинающейся и заканчивающейся в $A$ (см. рис. 2).

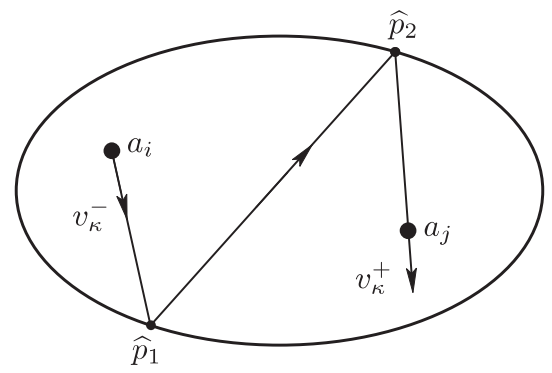

Рис. 2. Орбита бильярда в $\Omega_{0}$

Рассмотрим бильярдную траекторию $\kappa$ в $D$, начинающуюся и заканчивающуюся в точках $a_{i}, a_{j} \in A$. Она может быть отрезком $\kappa=\left(a_{i}, a_{j}\right)$, соединяющим $a_{i}$ и $a_{j}$, или ломаной

$$
\kappa=\left(a_{i}, p_{1}, \ldots, p_{n}, a_{j}\right), \quad p_{1}, \ldots, p_{n} \in \Sigma,
$$


соединяющей точки $a_{i}, p_{1}, \ldots, p_{n}, a_{j}$. Тогда $\left(p_{1}, \ldots, p_{n}\right)-$ критическая точка функции длины

$$
l\left(a_{i}, p_{1}, \ldots, p_{n}, a_{j}\right)=\left|a_{i}-p_{1}\right|+\left|p_{1}-p_{2}\right|+\cdots+\left|p_{n-1}-p_{n}\right|+\left|p_{n}-a_{j}\right|
$$

на $\Sigma^{n}$. Траектория $\kappa$ называется невырожденной, если $n=0$ или критическая точка $\left(p_{1}, \ldots, p_{n}\right)$ невырождена. Мы называем ломаную (5.3) квазитраекторией, если она невырождена и, кроме конечных точек $a_{i}$ и $a_{j}$, не содержит точек из множества $A$.

Мы будем использовать обозначения $a_{\kappa}^{-}=a_{i}$ и $a_{\kappa}^{+}=a_{j}$ для начальной и конечной точек квазитраектории, а также $v_{\kappa}^{-}$и $v_{\kappa}^{+}$для ее начального и конечного векторов скорости, выходящих из $a_{i}$ и $a_{j}$ соответственно:

$$
v_{\kappa}^{-}=\frac{p_{1}-a_{i}}{\left|p_{1}-a_{i}\right|}, \quad v_{\kappa}^{+}=\frac{a_{j}-p_{n}}{\left|a_{j}-p_{n}\right|} .
$$

В случае $n=0$ следует взять $v_{\kappa}^{-}=v_{\kappa}^{+}=\left(a_{j}-a_{i}\right) /\left|a_{j}-a_{i}\right|$.

Если $D$ выпуклая, то всегда существуют невырожденные квазитраектории с $n=0$, соединяющие $a_{i}$ и $a_{j}$. Также всегда существуют квазитраектории с $n=1$, соответствующие минимуму $l$ на $\Sigma$. В общем случае они невырождены.

При $m=2$, используя те же аргументы, что и в теореме Биркгофа [13] о периодических траекториях выпуклого бильярда (см. также [55]), легко показать, что если $D$ выпукла, то для любого $n \geqslant 1$ имеется не меньше $2 n$ орбит (5.3).

Любая невырожденная траектория (5.3) гладко деформируется, когда ее начальная и конечная точки слегка изменяются. Поэтому при малых $\varepsilon \geqslant 0$ существуют гладкие функции

$$
p_{1}(x, y), \ldots, p_{n}(x, y), \quad x, y \in S^{m-1},
$$

такие, что

$$
B_{\varepsilon}(x, y)=\left(a_{i}+\varepsilon \phi_{i}(x), p_{1}(x, y), \ldots, p_{n}(x, y), a_{j}+\varepsilon \phi_{j}(y)\right)
$$

- траектория бильярда в $D$, совпадающая с $\kappa$ при $\varepsilon=0$. Эта траектория содержится в $\Omega_{\varepsilon}$, если ее общими точками с $\partial \Omega_{\varepsilon}$ являются только конечные точки $a_{i}+\varepsilon q_{i}(x)$ и $a_{j}+\varepsilon q_{j}(y)$. Это выполняется, если $\varepsilon$ достаточно мало и $x \in U_{\kappa}^{-}$, $y \in U_{\kappa}^{+}$, где $U_{\kappa}^{ \pm} \subset S^{m-1}$ - некоторые открытые множества (см. рис. 3). Например, $U_{\kappa}^{-}-$множество точек $x$ таких, что луч, выходящий из $\phi_{i}(x)$ в направлении $v_{\kappa}^{-}$, не пересекает $\Sigma_{i}$.

Дискретный лагранжиан, соответствующий $\kappa$, имеет вид

$$
\begin{aligned}
L_{\kappa}(x, y)= & \varepsilon^{-1} l\left(B_{\varepsilon}\right) \\
= & \varepsilon^{-1}\left|a_{i}-p_{1}(x, y)+\varepsilon \phi_{i}(x)\right|+\varepsilon^{-1}\left|p_{1}(x, y)-p_{2}(x, y)\right|+\cdots \\
& \quad+\varepsilon^{-1}\left|p_{n-1}(x, y)-p_{n}(x, y)\right|+\varepsilon^{-1}\left|p_{n}(x, y)-a_{j}-\varepsilon \phi_{j}(y)\right| .
\end{aligned}
$$

Покажем, что

$$
L_{\kappa}(x, y)=\varepsilon^{-1} l(\kappa)+\left\langle v_{\kappa}^{+}, \phi_{i}(x)\right\rangle-\left\langle v_{\kappa}^{-}, \phi_{j}(y)\right\rangle+O(\varepsilon), \quad(x, y) \in S^{m-1} \times S^{m-1} .
$$




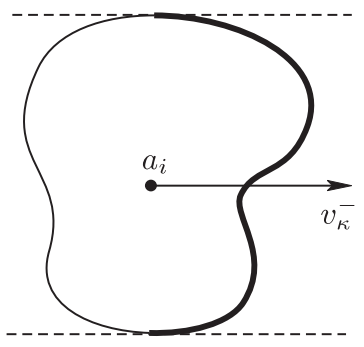

Рис. 3. Выделенная жирным часть границы $\partial D_{i}-$ множество $a_{i}+\varepsilon \phi_{i}\left(U_{\kappa}^{-}\right)$

Действительно, согласно формуле гамильтоновой первой вариации,

$$
d l\left(B_{\varepsilon}\right)=v_{\kappa}^{+} d q_{+}-v_{\kappa}^{-} d q_{-}, \quad d q_{-}=\phi_{i}(x) d \varepsilon, \quad d q_{+}=\phi_{j}(y) d \varepsilon .
$$

Следовательно,

$$
\left.\frac{\partial}{\partial \varepsilon}\right|_{\varepsilon=0} l\left(B_{\varepsilon}\right)=\left\langle v_{\kappa}^{+}, \phi_{j}(y)\right\rangle-\left\langle v_{\kappa}^{-}, \phi_{i}(x)\right\rangle .
$$

Пусть множество вершин графа Г есть конечный набор квазитраекторий $J=\{\kappa\}$. Вершины $\kappa$ и $\kappa^{\prime}$ соединяются ребром, если

1) конец $\kappa$ является началом $\kappa^{\prime}: a_{\kappa}^{+}=a_{\kappa^{\prime}}^{-}=a_{k} \in A$,

2) направление меняется: $v_{\kappa}^{+} \neq v_{\kappa^{\prime}}^{-}$.

Если $\Sigma_{k}$ строго выпукла, других условий не требуется. Если нет, потребуется еще одно. Пусть $v \in \mathbb{R}^{m}$ - единичный вектор

$$
v=\frac{v_{\kappa^{\prime}}^{-}-v_{\kappa}^{+}}{\left|v_{\kappa^{\prime}}^{-}-v_{\kappa}^{+}\right|},
$$

(см. рис. 4). Всегда найдется точка $s \in S^{m-1}$ такая, что $v$ есть внешняя нормаль к касательной плоскости $T_{\phi_{k}(s)} \Sigma_{k}$ и $s \in U_{\kappa}^{+} \cap U_{\kappa^{\prime}}^{-}$.



Рис. 4. Пульсирующая траектория, отслеживающие гомоклиники $\gamma_{k}$

3) Предполагается, что вторая фундаментальная форма $\left\langle v, d^{2} \phi_{k}(s)\right\rangle$ поверхности $\Sigma_{k}$ невырождена в $\phi_{k}(s)$.

Условие 3 ) всегда выполняется, если $\Sigma_{k}$ - строго выпуклая; тогда $s$ существует, единственна и получается максимизацией $\left\langle\phi_{k}(s), v\right\rangle$. Если же $\Sigma_{k}$ невыпукла, то таких точек $s$ может быть несколько, и тогда будем иметь несколько ребер $\gamma$, соединяющих вершины $\kappa, \kappa^{\prime}$. 
Итак, мы определили граф с множеством вершин $J=\{\kappa\}$ и множеством ребер $E=\{\gamma\}$. Лагранжиан $L_{\kappa}$ имеет антиинтегрируемый вид с $V_{\kappa}^{+}=\left\langle v_{\kappa}^{+}, \phi_{j}\right\rangle$ и $V_{\kappa}^{-}=-\left\langle v_{\kappa}^{-}, \phi_{i}\right\rangle$. Любой путь на графе определяет код. Теперь из теоремы 5 вытекает следующая теорема.

Теорема 7 (ср. с [30]). Пусть в достаточно мало. Тогда для любого кода $\mathbf{k}$ существует бильярдная траектория $\mathbf{x}$ в $\Omega_{\varepsilon}$, отслеживающая цепочку квазитраекторий $\mathbf{k}=\left(\kappa_{i}\right)$. Орбита $\mathbf{x}$ гиперболична.

Бильярд в $\Omega_{\varepsilon}$ является объемлющей системой для построенной ДЛС. Нижние оценки для топологической энтропии объемлющей системы можно вывести с помощью полученной топологической марковской цепи. Более подробный анализ задачи о топологической энтропии в бильярде с малыми рассеивателями дан в [31].

Отметим, что если рассеиватели выпуклые, то при некоторых условиях бильярд будет гиперболическим, так что имеют место намного более сильные результаты, например положительность метрической энтропии [28].

5.4. Теорема Биркгофа-Смейла-Шильникова. В качестве примера применения метода антиинтегрируемого предела для исследования симплектических отображений докажем существование хаотического гиперболического инвариантного множества для дискретной динамической системы, имеющей трансверсальную гомоклиническую траекторию. Эта теорема восходит к работам Дж. Д. Биркгофа [13] и С. Смейла [65], а в окончательной форме доказана Л. П. Шильниковым [63] (см. также [52], [64]).

Теорема Биркгофа-Смейла-Шильникова будет доказана для симплектических отображений, однако общее (несимплектическое) отображение сводится к симплектическому отображению удвоением размерности (см. замечание 8 в конце этого пункта).

Пусть $F: P \rightarrow P$ - симплектическое отображение $2 m$-мерного симплектического многообразия, имеющее гиперболическую неподвижную точку $O$. Предположим, что существуют трансверсальные гомоклинические траектории к точке $O$, и выберем конечное множество таких траекторий $\left\{\gamma_{k}\right\}_{k \in I}$. Мы покажем, что траектории отображения $F$, которые не покидают окрестность гомоклинического множества $\bigcup_{k \in I} \gamma_{k} \cup O$, могут быть представлены как траектории антиинтегрируемой ДЛС. Отсюда получается символическое представление траекторий в окрестности гомоклинического множества.

Будет удобно обозначить через $W^{+}$и $W^{-}$устойчивое и неустойчивое многообразия ${ }^{6}$ неподвижной точки $O$ :

$$
W^{ \pm}=\left\{x: F^{n}(x) \rightarrow O \text { при } n \rightarrow \pm \infty\right\} .
$$

Существуют симплектические координаты $q, p$ в окрестности $D$ точки $O$ такие, что $O=(0,0)$ и локальные устойчивое и неустойчивое многообразия $W_{\mathrm{loc}}^{ \pm} \subset D$

\footnotetext{
${ }^{6}$ Стандартное обозначение $-W^{s}$ и $W^{u}$.
} 
являются лагранжевыми графиками над шаром $U \subset \mathbb{R}^{m}$ :

$$
\begin{aligned}
& W_{\mathrm{loc}}^{+}=\left\{(q, p): q \in U, p=-\nabla S_{+}(q)\right\}, \\
& W_{\mathrm{loc}}^{-}=\left\{(q, p): q \in U, p=\nabla S_{-}(q)\right\} .
\end{aligned}
$$

Обозначим через

$$
\lambda_{j}, \lambda_{j}^{-1}, \quad 0<\left|\lambda_{j}\right|<1, \quad j=1, \ldots, m,
$$

собственные значения оператора $D F(O)$. Зафиксируем

$$
\alpha \in\left(\max \left\{\left|\lambda_{j}\right|\right\}, 1\right) .
$$

Локальное поведение траекторий отображения около точки $O$ описывается следующим утверждением, которое можно вывести из леммы Шильникова [63] или сильной $\lambda$-леммы [39].

Лемма 3. Пусть $N$ достаточно велико. Тогда для любых $n \geqslant N u q^{ \pm} \in U$ существуют $p^{ \pm}$такие, что $F^{n}\left(q^{+}, p^{+}\right)=\left(q^{-}, p^{-}\right)$u $F^{i}\left(q^{+}, p^{+}\right) \in D$ при $0 \leqslant$ $i \leqslant n$. Отображсене $\left(q^{+}, p^{+}\right) \mapsto\left(q^{-}, p^{-}\right)$- симплектический дифбеоморфизм $F^{n}: D_{n}^{+} \rightarrow D_{n}^{-}$открытых подмножеств в $D$, задаваемый производящей функиией (определенной с точностъю до константь)

$$
S_{n}\left(q^{+}, q^{-}\right)=S_{+}\left(q^{+}\right)+S_{-}\left(q^{-}\right)+u_{n}\left(q^{+}, q^{-}\right),
$$

где $\left\|u_{n}\right\|_{C^{2}(U \times U)} \leqslant C \alpha^{n}$.

Таким образом,

$$
F^{n}\left(q^{+}, p^{+}\right)=\left(q^{-}, p^{-}\right) \Longleftrightarrow p^{+} d q^{+}-p^{-} d q^{-}=d S_{n}\left(q^{+}, q^{-}\right) .
$$

Производящая функция $S_{n}$ локального отображения $F^{n}: D_{n}^{+} \rightarrow D_{n}^{-}$при больших $n$ имеет антиинтегрируемую форму. Чтобы получить хаотическую динамику, требуется вернуться в окрестность неподвижной точки $O$ с помощью глобального отображения вдоль гомоклинической траектории.

Пусть $\gamma_{k}$ - трансверсальная гомоклиническая траектория неподвижной точки $О$. Выберем произвольные точки $z_{k}^{ \pm}=\left(q_{k}^{ \pm}, p_{k}^{ \pm}\right) \in \gamma_{k} \cap W_{\text {loc }}^{ \pm}$. Найдется целое число $m_{k}$ такое, что $F^{m_{k}}\left(z_{k}^{-}\right)=z_{k}^{+}$. Отображение $F^{m_{k}}$ из окрестности $G_{k}^{-}$ точки $z_{k}^{-}$в окрестность $G_{k}^{+}$точки $z_{k}^{+}$назовем глобальным отображением. Поскольку это отображение симплектическое, существуют функции $\Phi_{k}$ такие, что

$$
F^{m_{k}}\left(q^{-}, p^{-}\right)=\left(q^{+}, p^{+}\right) \Longleftrightarrow p^{+} d q^{+}-p^{-} d q^{-}=d \Phi_{k} .
$$

Пошевелив, если потребуется, координаты, можно предположить, что

$$
\operatorname{det}\left(\frac{\partial q^{+}}{\partial p^{-}}\right) \neq 0 .
$$

Тогда можно локально выразить $\Phi_{k}$ как функцию $\Phi_{k}\left(q^{-}, q^{+}\right)$на $U_{k}^{-} \times U_{k}^{+}$, где $U_{k}^{ \pm} \subset U-$ окрестность точки $q_{k}^{ \pm}$. Тогда $\Phi_{k}$ является производящей функцией глобального отображения $F^{m_{k}}: G_{k}^{-} \rightarrow G_{k}^{+}$. 
Соотношения (5.4) и (5.6) означают, что функция

$$
R_{k}\left(q^{-}, q^{+}\right)=S_{-}\left(q^{-}\right)+\Phi_{k}\left(q^{-}, q^{+}\right)+S_{+}\left(q^{+}\right)
$$

имеет критическую точку $\left(q_{k}^{-}, q_{k}^{+}\right)$, которая соответствует гомоклинической траектории $\gamma_{k}$. Поскольку $\gamma_{k}$ трансверсальна, критическая точка является невырожденной.

Траектории отображения $F$, не покидающие окрестность гомоклинического множества, соответствуют траекториям ДЛС с лагранжианами $\left\{\Phi_{k}, S_{n}\right\}_{k \in I, n \geqslant N}$. Однако формально эта система не является антиинтегрируемой. Применение теоремы 5 упрощается, если рассмотреть другую ДЛС с $2 m$ степенями свободы.

Положим $J=\{\kappa=(k, n): k \in I, n \geqslant N\}$. Определим ДЛС $\left\{L_{\kappa}\right\}_{\kappa \in J}$ с дискретным лагранжианом $L_{\kappa}$, представляющим композицию $F^{n} \circ F^{m_{k}}$ глобального и локального отображений. Положим

$L_{\kappa}(x, y)=\Phi_{k}(x)+S_{n}\left(x^{+}, y^{-}\right), \quad x=\left(x^{-}, x^{+}\right) \in U_{k}^{-} \times U_{k}^{+}, \quad y=\left(y^{-}, y^{+}\right) \in U^{2}$.

ЗАмечАниЕ 7 . Лагранжиан $L_{\kappa}$ имеет $2 m$ степеней свободы. Его можно заменить приведенным лагранжианом

$$
\widetilde{L}_{\kappa}\left(x^{-}, y^{-}\right)=\operatorname{Crit}_{x^{+}}\left(\Phi_{k}\left(x^{-}, x^{+}\right)+S_{n}\left(x^{+}, y^{-}\right)\right)
$$

c $m$ степенями свободы. Это потребует дополнительного условия невырожденности, выполнения которого можно добиться, пошевелив координаты.

Траектории отображения $F$, отслеживающие цепочки гомоклинических траекторий $\left(\gamma_{k_{i}}\right)$, соответствуют критическим точкам функционала действия

$$
A_{\mathbf{k}}(\mathbf{x})=\sum L_{\kappa_{i}}\left(x_{i}, x_{i+1}\right), \quad x_{i}=\left(x_{i}^{-}, x_{i}^{+}\right) \in U_{k_{i}}^{-} \times U_{k_{i}}^{+}, \quad \kappa_{i}=\left(k_{i}, n_{i}\right) .
$$

Чтобы получить антиинтегрируемую ДЛС, достаточно заменить $L_{\kappa}$ на калибровочно эквивалентный лагранжиан (с тем же функционалом действия)

$$
\widehat{L}_{\kappa}(x, y)=L_{\kappa}(x, y)+S_{-}\left(x^{-}\right)-S_{-}\left(y^{-}\right)=R_{k}(x)+O\left(\alpha^{n}\right) .
$$

Функция $R_{k}$ имеет невырожденную критическую точку $\left(q_{k}^{-}, q_{k}^{+}\right)$. В обозначениях теоремы 5 имеем антиинтегрируемую ДЛС, задаваемую графом $Г$ с множеством вершин $J$, и каждые две вершины соединены ребром. При отсутствии ограничения сверху на $n_{i}$ граф Г является бесконечным, но легко проверить, что теорема 6 применима. Доказана теорема Биркгофа-Смейла-Шильникова.

Теорема 8. Для каждого кода $\kappa_{i}=\left(k_{i}, n_{i}\right) \in J$ соответствующая гомоклиническая иепочка $\left(\gamma_{k_{i}}\right)$ отслеживается единственной гиперболической траекторией отображения $F$, которая отслеживает $\gamma_{k_{i}}$, затем в течение $n_{i}$ итераций отображения $F$ движется в окрестности точки $O$, затем отслеживает $\gamma_{k_{i+1}}$ и т.д. Таким образом, $F$ имеет хаотическое гиперболическое инвариантное множество. 
Обычно для символического описания динамики в окрестности гомоклинического множества используется немного другой граф [52]: его вершины соответствуют гомоклиническим траекториям $\gamma_{k}$ и еще одна вершина отвечает неподвижной точке $O$. Путь на графе после вершины $\gamma_{k_{i}}$ остается несколько шагов $n_{i}$ на вершине $O$ прежде чем следовать к вершине $\gamma_{k_{i+1}}$. Таким образом, путь соответствует последовательности $\left(k_{i}, n_{i}\right)$, как и в теореме 8 , так что символическая динамика такая же.

ЗАмечАниЕ 8. Теорема Биркгофа-Смейла-Шильникова для общих (несимплектических) отображений $f$ вытекает из теоремы 8. Действительно, отображение $q^{+}=f\left(q^{-}\right)$сводится к симплектическому отображению $F$ с производящей функцией $S\left(q^{-}, p^{+}\right)=\left\langle f\left(q^{-}\right), p^{+}\right\rangle$удвоением размерности:

$$
p^{-}=\partial_{q^{-}} S, \quad q^{+}=\partial_{p^{+}} S=f\left(q^{-}\right) .
$$

Если $f$ имеет гиперболическую неподвижную точку, обладающую трансверсальными гомоклиническими траекториями, то то же верно для $F$. Таким образом, теорема 8 доказана для несимплектических отображений.

\section{5. Цепочки гетероклинических траекторий инвариантных торов.}

Следующий пример применения метода антиинтегрируемого предела основан на работе [15]. Предположим, что симплектический диффеоморфизм $F: P \rightarrow P$ имеет $d$-мерный гиперболический инвариантный тор $Г$. Тогда $\Gamma$ - образ гладкого вложения $h: \mathbb{T} \rightarrow \mathbb{P}$ и $\left.F\right|_{\Gamma}$ сопряжено сдвигу с вектором вращения $\rho \in \mathbb{R}^{d}$ :

$$
F(h(x))=F(x+\rho) .
$$

Если вектор вращения диофантов, т. е.

$$
\left|\langle\rho, j\rangle-j_{0}\right| \geqslant \alpha|j|^{-\beta}, \quad \alpha, \beta>0,
$$

для любых $j \in \mathbb{Z}^{d} \backslash\{0\}$ и $j_{0} \in \mathbb{Z}$, то говорят, что тор диофантов. Будем предполагать также, что тор изотропен, т.е. $\left.\omega\right|_{\Gamma}=0$. Если тор диофантов и симплектическая структура точная, это всегда так.

ОПРЕДЕлЕниЕ 2. Тор Г называется гиперболическим, если существуют гладкие $(m-d)$-мерные подрасслоения $E^{ \pm}$в касательном расслоении $T_{\Gamma} P$ такие, что:

(i) $E^{ \pm}$инвариантны относительно линеаризованного отображения $D F$, т. е.

$$
D F(x) E_{x}^{ \pm}=E_{F(x)}^{ \pm} \quad \text { для всех } x \in \Gamma ;
$$

(ii) линеаризованное отображение является сжатием на $E^{+}$и растяжением на $E^{-}$, т. е. найдутся константы $c>0, \lambda>1$ такие, что для всех $x \in \Gamma$

$$
\left\|\left.D F^{k}(x)\right|_{E_{x}^{+}}\right\| \leqslant c \lambda^{-k}, \quad\left\|\left.D F^{-k}(x)\right|_{E_{x}^{-}}\right\| \leqslant c \lambda^{-k}, \quad k \in \mathbb{N} .
$$

Здесь $\|\cdot\|$ - норма, задаваемая некоторой римановой метрикой. Поскольку тор Г компактен, определение не зависит от метрики. 
ОПредЕЛЕНиЕ 3. Тор Г называется невырожденным, если ограниченные траектории линеаризованного отображения касаются Г. Таким образом, если $x \in \Gamma, v \in T_{x} M$ и $\left\|D F^{k}(x) v\right\| \leqslant c$ для всех $k \in \mathbb{Z}$, то $v \in T_{x} \Gamma$.

Определение гиперболического тора можно переписать в координатной форме следующим образом.

ОПРЕДЕЛЕНИЕ 4. Инвариантный тор Г называется гиперболическим, если в его трубчатой окрестности $D$ существуют симплектические координаты $x \in \mathbb{T}^{d}$, $y \in \mathbb{R}^{d}, z_{ \pm} \in \mathbb{R}^{m-d}$ такие, что:

(a) $\left.\omega\right|_{D}=d y \wedge d x+d z_{+} \wedge d z_{-}$;

(b) $\Gamma$ задается уравнениями $y=0, z_{-}=z_{+}=0$;

(c) отображение $\left.F\right|_{D}$ имеет вид

$$
\left(\begin{array}{c}
x \\
y \\
z_{-} \\
z_{+}
\end{array}\right) \mapsto\left(\begin{array}{c}
x+\rho+A y \\
y \\
B^{*}(x)^{-1} z_{-} \\
B(x) z_{+}
\end{array}\right)+O_{2}\left(y, z_{-}, z_{+}\right)
$$

(d) показатели Ляпунова косого произведения $(x, z) \mapsto(x+\rho, B(x) z)$ отрицательны: существует норма такая, что $\|B(x)\| \leqslant \alpha<1$;

(е) симметрическая матрица $A$ постоянна.

Для диофантова изотропного тора определения 2 и 4 эквивалентны (см. [25]). Если используется определение 4, то для дальнейшего не требуется предполагать, что тор диофантов. Однако в приложениях гиперболические инвариантные торы обычно диофантовы. Гиперболический тор невырожден, если $\operatorname{det} A \neq 0$. Согласно KAМ-теории гиперболические невырожденные диофантовы инвариантные торы сохраняются при малых гладких точных симплектических возмущениях отображения.

Гиперболический тор Г имеет $m$-мерные устойчивое $W^{+}$и неустойчивое $W^{-}$многообразия, состоящие из траекторий $F^{n}(z)$, асимптотических к Г при $n \rightarrow+\infty$ и $n \rightarrow-\infty$ соответственно.

Предположим, что $\left\{\Gamma_{k}\right\}_{k \in I}-$ конечное множество невырожденных гиперболических торов размерности $0<d_{k}<m$, а $W_{k}^{ \pm}-$их устойчивые и неустойчивые многообразия. Точка $z \in W_{j}^{-} \cap W_{k}^{+}$называется гетероклинической точкой, а ее траектория $\gamma=\left(F^{n}(z)\right)_{n \in \mathbb{Z}}$ - гетероклинической траекторией, соединяющей $\Gamma_{j}$ с $\Gamma_{k}$. Такая траектория называется трансверсальной, если $T_{z} W_{j}^{-} \cap T_{z} W_{k}^{+}=\{0\}$.

Пусть $\left\{\gamma_{\kappa}\right\}_{\kappa \in J}-$ конечное множество трансверсальных гетероклинических ${ }^{7}$ траекторий, соединяющих пары торов из множества $\left\{\Gamma_{k}\right\}_{k \in I}$. Обозначим через $G$ ориентированный граф с вершинами $k \in I$ и ребрами $\kappa \in J$, соответствующими гетероклиническим траекториям.

Теорема 9. Пусть $N>0$ достаточно велико и дана последовательность целых чисел $\left(n_{i}\right)_{i \in \mathbb{Z}}, n_{i} \geqslant N$. Для любого пути $\kappa=\left(\kappa_{i}\right)_{i \in \mathbb{Z}}$ на графе $G$, соответствующего последовательности $\left(\gamma_{\kappa_{i}}\right)_{i \in \mathbb{Z}}$ гетероклинических траекторий, соединяющих $\Gamma_{k_{i-1}} u \Gamma_{k_{i}}$, существует (не единственная) траектория

\footnotetext{
${ }^{7}$ В дальнейшем гетероклиническая означает гетероклиническая или гомоклиническая.
} 
отображения $F$, отслеживающая гетероклиническую иепочку $\left(\gamma_{\kappa_{i}}\right)_{i \in \mathbb{Z}}$. После отслеживания $\gamma_{\kappa_{i}}$ она в течение $n_{i}$ итераций отображения $F$ остается в окрестности тора $\Gamma_{k_{i}}$.

Подобные утверждения обычно доказываются методом окон Истона [46], однако доказательство с помощью антиинтегрируемого предела проще и более конструктивное. Альтернативный подход основан на вариационных методах (см., например, [58], [49], [11], [32], [33], [50]), однако эти методы работают лишь для положительно определенных лагранжевых систем.

Теорема 9 аналогична лемме о цепочках в общей теории гиперболических множеств динамических систем [52]. Разница в том, что множество $\bigcup_{k \in I} \Gamma_{k} \cup$ $\bigcup_{\kappa \in J} \gamma_{\kappa}$ не гиперболическое, так что стандартные теоремы гиперболической теории неприменимы. Теорема 9 может быть использована для построения диффузионных траекторий в задаче о диффузии Арнольда для $\varepsilon$-малых возмущений априори неустойчивой интегрируемой системы (см. п. 7.2). Однако такой подход возможен только при отсутствии сильных резонансов и, кроме того, с помощью теоремы 9 получается нижняя оценка скорости диффузии порядка $O\left(\varepsilon^{2}\right)$, что гораздо слабее оценки скорости диффузии $O(\varepsilon /|\log \varepsilon|)$, получаемой при использовании метода антиинтегрируемого предела для сепаратрисного отображения (см. п. 7.2).

Для 0-мерных гиперболических торов (гиперболических неподвижных точек) теорема 9 есть ослабленный вариант теоремы 8. Доказательства этих теорем аналогичны. Вместо леммы 3 будет использована приводимая ниже лемма 4.

Пусть Г - гиперболический тор. Выберем симплектические координаты $(q, p)$ в малой трубчатой окрестности $D$ тора $\Gamma$ так, что локальные устойчивое и неустойчивое лагранжевы многообразия задаются производящими функциями:

$$
\begin{aligned}
& W_{\mathrm{loc}}^{+}=\left\{(q, p) \in D: q \in U, p=-\nabla S^{+}(q)\right\}, \\
& W_{\mathrm{loc}}^{-}=\left\{(q, p) \in D: q \in U, p=\nabla S^{-}(q)\right\} .
\end{aligned}
$$

Проекция тора $\Gamma=W_{\text {loc }}^{+} \cap W_{\text {loc }}^{-}$на $U-$ тоже тор $T=\left\{q: \nabla\left(S^{+}+S^{-}\right)(q)=0\right\}$.

Поскольку $U$ - трубчатая окрестность тора $T$, имеется (неканоническая) проекция $U \rightarrow \mathbb{T}^{d}$. Обозначим через $\widetilde{U}$ универсальное накрытие окрестности $U$. Получим отображение $\phi: \widetilde{U} \rightarrow \mathbb{R}^{d}$. Диффеоморфизм $F$ поднимается до отображения $F: \widetilde{D} \rightarrow \widetilde{D}$.

Лемма 4. Зафиксируем $r>0$. Существует $N>0$ такое, что:

(а) для любого $n \geqslant N$ и всех $\left(q_{+}, q_{-}\right)$из множества

$$
Y^{n}=\left\{\left(q_{+}, q_{-}\right) \in \widetilde{U} \times \widetilde{U}:\left|\phi\left(q_{-}\right)-\phi\left(q_{+}\right)-n \rho\right| \leqslant r\right\}
$$

существуют единственнъе $z_{+}=\left(q_{+}, p_{+}\right) \in \widetilde{D} u z_{-}=\left(q_{-}, p_{-}\right) \in \widetilde{D}$ такие, что $F^{n}\left(z_{+}\right)=z_{-} u F^{j}\left(z_{+}\right) \in \widetilde{D} n p u j=0,1, \ldots, n$;

(b) отображение $F^{n}:\left(q_{+}, p_{+}\right) \rightarrow\left(q_{-}, p_{-}\right)$задается производящей функциeй $Q^{n}$ :

$$
F^{n}\left(q_{+}, p_{+}\right)=\left(q_{-}, p_{-}\right) \quad \Longleftrightarrow \quad p_{-}=\partial_{q_{-}} Q^{n}\left(q_{+}, q_{-}\right), \quad p_{+}=-\partial_{q_{+}} Q^{n}\left(q_{+}, q_{-}\right),
$$


(с) $Q^{n}$ является гладкой функцией на $Y^{n}$ и имеет вид

$$
Q^{n}\left(q_{+}, q_{-}\right)=S^{+}\left(q_{+}\right)+S^{-}\left(q_{-}\right)+n^{-1} v_{n}\left(q_{+}, q_{-}\right),
$$

где $\left\|v_{n}\right\|_{C^{2}\left(Y^{n}\right)} \leqslant C r^{2} ;$ константа $C>0$ не зависит от $n$.

Здесь не требуется, чтобы вектор $\rho$ был диофантовым или нерезонансным, но важно, что матрица $A$ невырожденная. Лемма 4 доказана в [15] для $d=m-1$. Для любого $0<d<m$ доказательство аналогично. При $d=0$ лемма 3 дает более точную оценку производящей функции.

Для каждого тора $\left\{\Gamma_{k}\right\}_{k \in I}$ определим трубчатые окрестности $U_{k}$ и $D_{k}$ и производящие функции $S_{k}^{ \pm}$и $Q_{k}^{n}$ так же, как выше. Производящая функция в лемме 4 является дискретным лагранжианом, описывающим локальное отображение около гиперболического тора $\Gamma_{k}$. Определим теперь дискретный лагранжиан, описывающий глобальное отображение в окрестности трансверсальной гетероклинической траектории $\gamma_{\kappa}$.

Возьмем два тора $\Gamma_{j}, \Gamma_{k}$, соединенные гетероклинической (гомоклинической, если $k=j$ ) траекторией $\gamma_{\kappa} \subset W_{j}^{-} \cap W_{k}^{+}$. Существуют точки

$$
z_{\kappa}^{-}=\left(q_{\kappa}^{-}, p_{\kappa}^{-}\right) \in \gamma_{\kappa} \cap D_{j}, \quad z_{\kappa}^{+}=\left(q_{\kappa}^{+}, p_{\kappa}^{+}\right) \in \gamma_{\kappa} \cap D_{k}
$$

и число $m_{\kappa} \in \mathbb{N}$ такие, что $F^{m_{\kappa}}\left(z_{\kappa}^{-}\right)=z_{\kappa}^{+}$.

Симплектические координаты можно выбрать таким образом, чтобы глобальное отображение $F^{m_{\kappa}}$ из окрестности точки $z_{\kappa}^{-}$в окрестность точки $z_{\kappa}^{+}$ задавалось производящей функцией $\Phi_{\kappa}$, определенной на множестве $X_{\kappa}^{-} \times X_{\kappa}^{+}$, где $X_{\kappa}^{ \pm}$- малая окрестность точки $q_{\kappa}^{ \pm}$:

$$
F^{m_{\kappa}}\left(q_{-}, p_{-}\right)=\left(q_{+}, p_{+}\right) \Longleftrightarrow p_{+}=\partial_{q_{+}} \Phi_{\kappa}\left(q_{-}, q_{+}\right), \quad p_{-}=-\partial_{q_{-}} \Phi_{\kappa}\left(q_{-}, q_{+}\right) .
$$

Поскольку гетероклиническая траектория $\gamma_{\kappa}$ является трансверсальной, то в точности так же, как в п. 5.4, получим, что $\left(q_{\kappa}^{-}, q_{\kappa}^{+}\right)$- невырожденная критическая точка функции

$$
R_{\kappa}\left(q_{-}, q_{+}\right)=S_{j}^{-}\left(q_{-}\right)+\Phi_{\kappa}\left(q_{-}, q_{+}\right)+S_{k}^{+}\left(q_{+}\right), \quad\left(q_{-}, q_{+}\right) \in X_{\kappa}^{-} \times X_{\kappa}^{+} .
$$

Обозначим через $\pi: \widetilde{U}_{k} \rightarrow U_{k}$ универсальное накрытие с группой накрывающих преобразований

$$
\tau_{v}: \widetilde{U}_{k} \rightarrow \widetilde{U}_{k}, \quad v \in \mathbb{Z}^{d_{k}}, \quad d_{k}=\operatorname{dim} \Gamma_{k} .
$$

Зафиксируем связные компоненты $Z_{\kappa}^{ \pm}$множества $\tilde{X}_{\kappa}^{ \pm}=\pi^{-1}\left(X_{\kappa}^{ \pm}\right)$. Тогда $\tilde{X}_{\kappa}^{ \pm}=$ $\bigcup \tau_{v} Z_{\kappa}^{ \pm}$. Чтобы уменьшить неединственность, предположим, что множе$v \in \mathbb{Z}^{d_{k}}$

ства $Z_{\kappa}^{ \pm}$пересекаются с одной и той же фундаментальной областью $K$ группового действия. Тогда

$$
\max \left\{d(x, y): x \in Y_{\kappa}^{+}, y \in Y_{\kappa}^{-}\right\} \leqslant 2 \operatorname{diam} K .
$$

Пусть $\rho_{k} \in \mathbb{R}^{d_{k}}$ - вектор вращения тора $\Gamma_{k}$. Зафиксируем $R>\sqrt{d_{k}}$. Для любого $n \in \mathbb{N}$ существует вектор $v \in \mathbb{Z}^{d_{k}}$ такой, что

$$
\left|v-n \rho_{k}\right| \leqslant R \text {. }
$$


Тогда

$$
\left|\phi(y)-\phi(x)-n \rho_{k}\right| \leqslant r=R+2 \operatorname{diam} K
$$

для всех $x \in Z_{\kappa}^{+}$и $y \in \tau_{v} Z_{\kappa}^{-}$. Пусть $N>0$ достаточно велико и $n \geqslant N$. Тогда

$$
Z_{\kappa}^{+} \times \tau_{v} Z_{\kappa}^{-} \subset Y_{k}^{n},
$$

где $Y_{k}^{n}$ - множество (5.8), соответствующее тору $\Gamma_{k}$.

Положим $\sigma=(\kappa, n, v)$, где $n \geqslant N$ и вектор $v$ - тот же, что в (5.12). Определим дискретный лагранжиан $L_{\sigma}$ формулой

$$
L_{\sigma}(x, y)=\Phi_{\kappa}(x)+Q_{k}^{n}\left(x_{+}, \tau_{v} y_{-}\right), \quad x=\left(x_{-}, x_{+}\right), \quad y=\left(y_{-}, y_{+}\right),
$$

где $Q_{k}^{n}$ - функция $(5.9)$, соответствующая тору $\Gamma_{k}$. Лагранжиан $L_{\sigma}$ описывает траектории, которые отслеживают гетероклиническую траекторию $\gamma_{\kappa}$, а затем в течение $n$ итераций отображения $F$ остаются в окрестности тора $\Gamma_{k}$. Как и в п. 5.4, лагранжиан $L_{\sigma}$ имеет $2 m$ степеней свободы, но, если требуется, его можно заменить лагранжианом с $m$ степенями свободы.

Чтобы получить антиинтегрируемый лагранжиан, сделаем калибровочное преобразование:

$$
\widehat{L}_{\sigma}(x, y)=L_{\sigma}(x, y)+S_{j}^{-}\left(x_{-}\right)-S_{k}^{-}\left(y_{-}\right) .
$$

Согласно $(5.9), \widehat{L}_{\sigma}=R_{\kappa}(x)+O\left(n^{-1}\right)$, где $R_{\kappa}$ имеет невырожденную критическую точку $\left(q_{\kappa}^{-}, q_{\kappa}^{+}\right)$.

Предположим, что дана цепочка гетероклинических траекторий $\left(\gamma_{\kappa_{i}}\right)_{i \in \mathbb{Z}}$, где $\gamma_{\kappa_{i}}$ соединяет $\gamma_{k_{i-1}}$ и $\gamma_{k_{i}}$. Рассмотрим бесконечное произведение

$$
Z=\prod_{i \in \mathbb{Z}} Z_{\kappa_{i}}^{-} \times Z_{\kappa_{i}}^{+}
$$

состоящее из последовательностей

$$
\mathbf{x}=\left(x_{i}\right), \quad x_{i}=\left(x_{i}^{-}, x_{i}^{+}\right) \in Z_{\kappa_{i}}^{-} \times Z_{\kappa_{i}}^{+} .
$$

Для любой последовательности $\left(n_{i}, v_{i}\right)$ такой, что $n_{i} \geqslant N$ и $\left|v_{i}-n_{i} \rho_{k_{i}}\right| \leqslant R$, положим $\sigma_{i}=\left(\kappa_{i}, n_{i}, v_{i}\right)$ и определим на $Z$ формальный функционал

$$
A_{\sigma}(\mathbf{x})=\sum_{i \in \mathbb{Z}} L_{\sigma_{i}}\left(x_{i}, x_{i+1}\right)=\sum_{i \in \mathbb{Z}} \widehat{L}_{\sigma_{i}}\left(x_{i}, x_{i+1}\right) .
$$

Его критические точки соответствуют траекториям, отслеживающим гетероклиническую цепочку $\left(\gamma_{\kappa_{i}}\right)$. Поскольку лагранжиан $\widehat{L}_{\sigma}$ антиинтегрируемый, теорема 9 вытекает из теоремы 5 .

\section{6. Антиинтегрируемый предел в непрерывных лагранжевых системах}

В предыдущем разделе были рассмотрены примеры применения антиинтегрируемого предела в дискретных динамических системах. В этом разделе даны применения метода антиинтегрируемого предела к непрерывным лагранжевым и гамильтоновым системам - два примера для автономных систем и один для неавтономных. 
6.1. Теорема Тураева-Шильникова. В качестве примера применения метода антиинтегрируемого предела будет доказана теорема Тураева-Шильникова [72] для автономных гамильтоновых систем, имеющих трансверсальную гомоклиническую траекторию к гиперболическому положению равновесия. Этот случай сложнее, чем для гиперболических неподвижных точек симплектического отображения: без дополнительных условий система может быть интегрируема [40]. Приведенное здесь доказательство следует работе [24].

Рассмотрим гамильтонову систему с функцией Гамильтона $H$ на симплектическом многообразии $P$. Обозначим через $\phi^{t}$ фазовый поток. Пусть $O-$ гиперболическое положение равновесия. Будем предполагать, что $H(O)=0$. Пусть

$$
\pm \lambda_{j}, \quad 0<\operatorname{Re} \lambda_{1} \leqslant \cdots \leqslant \operatorname{Re} \lambda_{m},
$$

обозначают собственные значения положения равновесия. Предположим, что собственное значение $\lambda_{1}$ с наименьшей вещественной частью вещественно и

$$
0<\lambda_{1}<\operatorname{Re} \lambda_{2}
$$

Случай комплексного собственного значения $\lambda_{1}$ чуть проще, он изучается в [40], [56], [27].

Обозначим через $v_{+}$собственный вектор, соответствующий собственному значению $-\lambda_{1}$, а через $v_{-}$собственный вектор, соответствующий собственному значению $\lambda_{1}$. Зафиксируем метрику и предположим, что $\left|v_{ \pm}\right|=1$. Чтобы уменьшить неединственность собственных векторов, предположим, что $\omega\left(v_{-}, v_{+}\right)>0$, где $\omega-$ симплектическая 2-форма. Тогда собственные векторы $v_{ \pm}$единственны с точностью до замены $\left(v_{+}, v_{-}\right) \mapsto\left(-v_{+},-v_{-}\right)$.

Пусть $W^{ \pm}$- соответственно устойчивое и неустойчивое многообразия положения равновесия $O$. Они содержат сильные устойчивое и неустойчивое многообразия $W^{++} \subset W^{+}$и $W^{--} \subset W^{-}$, соответствующие собственным значениям $\pm \lambda_{j}, j>1$. Любая траектория в $W^{+} \backslash W^{++}$касается вектора $v_{+}$при $t \rightarrow+\infty$, а любая траектория в $W^{-} \backslash W^{--}$касается вектора $v_{-}$при $t \rightarrow-\infty$.

Пусть $\gamma(t)=\phi^{t}(z)$ - гомоклиническая траектория: $\lim _{t \rightarrow \pm \infty} \gamma(t)=O$. Она называется трансверсальной, если $T_{\gamma(0)} W^{+} \cap T_{\gamma(0)} W^{-}=\mathbb{R} \dot{\gamma}(0)$. Тогда пересечение $W^{+}$и $W^{-}$вдоль $\gamma$ трансверсально на уровне энергии $H=0$.

Предположим, что существуют несколько трансверсальных гомоклинических траекторий $\left\{\gamma_{\kappa}\right\}_{\kappa \in J}$, которые не содержатся в сильных устойчивом и неустойчивом многообразиях положения равновесия $O$. Тогда $\gamma_{\kappa}$ касается $v_{ \pm}$при $t \rightarrow \pm \infty$ соответственно:

$$
\lim _{t \rightarrow \infty} \frac{\dot{\gamma}_{\kappa}(t)}{\left|\dot{\gamma}_{\kappa}(t)\right|}=s_{\kappa}^{ \pm} v_{ \pm}, \quad s_{\kappa}^{ \pm}=+ \text {или }-.
$$

Определим граф $\Gamma_{-}$следующим образом. Каждая вершина $\kappa \in J$ соответствует трансверсальной гомоклинической траектории $\gamma_{\kappa}$. Вершины $\kappa$ и $\kappa^{\prime}$ соединены ребром, если $s_{\kappa}^{+}=s_{\kappa^{\prime}}^{-}$. Граф $\Gamma_{+}$определяется аналогично, но $\kappa$ и $\kappa^{\prime}$ соединены ребром, если $s_{\kappa}^{+}=-s_{\kappa^{\prime}}^{-}$. 
Tеорема 10. Существует $\varepsilon_{0}>0$ mакое, что для любого $\varepsilon \in\left(0, \varepsilon_{0}\right)$ и любого пути $\mathbf{k}=\left(\kappa_{i}\right)_{i \in \mathbb{Z}}$ в графе $\Gamma_{+}$существует гиперболическая траектория с энергией $H=\varepsilon$, отслеживающая гомоклиническую иепочку $\left(\gamma_{\kappa_{i}}\right)$. Аналогично, для любого пути $\mathbf{k}=\left(\kappa_{i}\right)_{i \in \mathbb{Z}}$ в графе $\Gamma_{-}$цепочка $\left(\gamma_{\kappa_{i}}\right)$ отслеживается гиперболической траекторией с энергией $H=-\varepsilon$.

Для доказательства будет построена антиинтегрируемая ДЛС, описывающая отслеживающие траектории. Тогда остается применить теорему 5.

Существуют симплектические координаты $q, p$ в окрестности $D$ положения равновесия $O$ такие, что $O=(0,0)$ и локальные устойчивое и неустойчивое многообразия $W_{\text {loc }}^{ \pm}$являются лагранжевыми графиками над малым шаром $U \subset \mathbb{R}^{m}:$

$$
\begin{aligned}
& W_{\mathrm{loc}}^{+}=\left\{(q, p): q \in U, p=-\nabla S_{+}(q)\right\}, \\
& W_{\mathrm{loc}}^{-}=\left\{(q, p): q \in U, p=\nabla S_{-}(q)\right\} .
\end{aligned}
$$

Будем считать, что $S_{ \pm}(0)=0$. Можно предполагать, что координаты выбраны так, что сильные локальные устойчивое и неустойчивое многообразия имеют вид

$$
W_{\mathrm{loc}}^{++}=\left\{(q, p) \in W_{\mathrm{loc}}^{+}: q_{1}=0\right\}, \quad W_{\mathrm{loc}}^{--}=\left\{(q, p) \in W_{\mathrm{loc}}^{-}: q_{1}=0\right\}
$$

Зафиксируем малое $\delta>0$ и для $s=+$ или $s=-$ положим

$$
U_{s}=\left\{q \in U: s q_{1}>\delta|q|\right\}, \quad W_{s}^{ \pm}=\left\{(q, p) \in W_{\mathrm{loc}}^{ \pm}: q \in U_{s}\right\} .
$$

Выберем знаки таким образом, чтобы векторы $v_{ \pm}$были направлены в сторону $W_{+}^{ \pm}$соответственно. Тогда гомоклиническая траектория $\gamma_{\kappa}$ удовлетворяет условию $\gamma_{\kappa}(t) \in W_{s_{\kappa}^{ \pm}}^{ \pm}$при $t \rightarrow \pm \infty$.

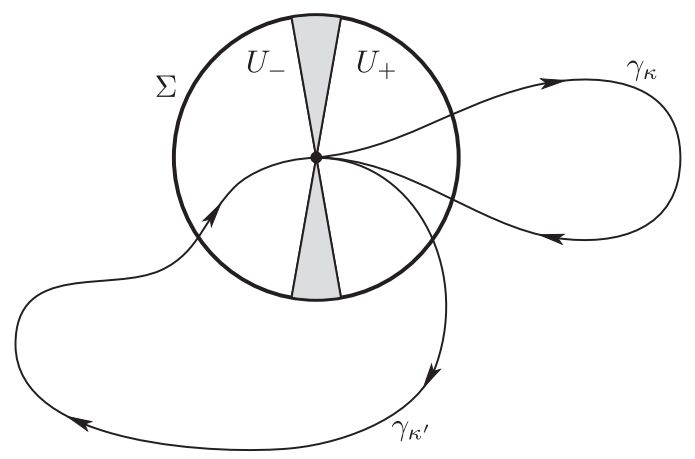

Рис. 5. Гомоклиники $\gamma_{\kappa}$ и $\gamma_{\kappa^{\prime}}$ с $s_{\kappa}^{-}=s_{\kappa}^{+}=s_{k^{\prime}}^{-}=+1$ и $s_{\kappa^{\prime}}^{+}=-1$

Зафиксируем $\alpha \in(0,1)$.

Лемма 5. Пусть $\varepsilon_{0}>0$ достаточно мало $u \varepsilon \in\left(0, \varepsilon_{0}\right)$. Пусть $s=+$ или $s=-$. Для любого $q_{ \pm} \in U_{s}$ существуют $p_{ \pm} u \tau>0$ mакие, ито $H\left(q_{ \pm}, p_{ \pm}\right)=-\varepsilon$ и $\phi^{\tau}\left(q_{+}, p_{+}\right)=\left(q_{-}, p_{-}\right)$, причем траектория $\gamma(t)=\phi^{t}\left(q_{+}, p_{+}\right), 0 \leqslant t \leqslant \tau$, 
остается в области $D$ при $0 \leqslant t \leqslant \tau$. Действие Мопертюи траектории $\gamma$ имеет вид

$$
S\left(q_{+}, q_{-}, \varepsilon\right)=\int_{\gamma} p d q=S_{+}\left(q_{+}\right)+S_{-}\left(q_{-}\right)+w\left(q_{+}, q_{-}, \varepsilon\right),
$$

где $\|w\|_{C^{2}} \leqslant C \varepsilon^{\alpha}$. Если $q_{+} \in U_{s} u q_{-} \in U_{-s}$, то справедливо аналогичное утверждение, но траектория $\gamma$ имеет энергию $H=\varepsilon$.

Поэтому для малых $\varepsilon>0$ траектории, проходящие вблизи точки $O$, описываются антиинтегрируемым дискретным лагранжианом. Лемма 5 доказана в работе [24].

ДОКАЗАТЕЛЬСТВо ТЕОРЕмЫ 10. Для определенности рассмотрим случай отслеживающих траекторий с отрицательной энергией $H=-\varepsilon$.

Положим $\Sigma=\partial U$. Пусть гомоклиническая траектория $\gamma_{\kappa}$ покидает $W_{\text {loc }}^{-}$ в точке $\left(q_{\kappa}^{+}, p_{\kappa}^{+}\right)$и входит в $W_{\text {loc }}^{+}$в точке $\left(q_{\kappa}^{+}, p_{\kappa}^{+}\right)$, где $q_{\kappa}^{ \pm} \in \Sigma$. Тогда найдется $t_{\kappa}>0$ такое, что $\phi^{t_{\kappa}}\left(q_{\kappa}^{-}, p_{\kappa}^{-}\right)=\left(q_{\kappa}^{+}, p_{\kappa}^{+}\right)$. Будем предполагать, что $\operatorname{det}\left(\partial q_{+} / \partial p_{-}\right) \neq 0$. Тогда существуют окрестности $X_{\kappa}^{ \pm}$точек $q_{\kappa}^{ \pm}$в $\Sigma$ такие, что для любых $\left(q_{-}, q_{+}\right) \in X_{\kappa}^{-} \times X_{\kappa}^{+}$и любого $\varepsilon \in\left(0, \varepsilon_{0}\right)$ существуют $p_{ \pm}$такие, что $H\left(q_{ \pm}, p_{ \pm}\right)=-\varepsilon$ и траектория $\phi^{t}\left(q_{-}, p_{-}\right), 0 \leqslant t \leqslant \tau=t_{\kappa}+O(\varepsilon)$, с энергией $-\varepsilon$ обладает свойством $\phi^{\tau}\left(q_{-}, p_{-}\right)=\left(q_{+}, p_{+}\right)$. Отображение $\left(q_{-}, p_{-}\right) \mapsto\left(q_{+}, p_{+}\right)$ имеет производящую функцию $\Phi_{\kappa}\left(q_{-}, q_{+}, \varepsilon\right)$ такую, что $d \Phi_{\kappa}=p_{+} d q_{+}-p_{-} d q_{-}$.

При $\varepsilon=0$ из (6.1) вытекает, что функция

$$
R_{\kappa}\left(q_{-}, q_{+}\right)=S_{-}\left(q_{-}\right)+\Phi_{\kappa}\left(q_{-}, q_{+}, 0\right)+S_{+}\left(q_{+}\right), \quad\left(q_{-}, q_{+}\right) \in X_{\kappa}^{-} \times X_{\kappa}^{+},
$$

имеет критическую точку $\left(q_{\kappa}^{-}, q_{\kappa}^{+}\right)$, соответствующую гомоклинической траектории $\gamma_{\kappa}$. Поскольку траектория $\gamma_{\kappa}$ трансверсальная, критическая точка невырожденная.

При малых $\varepsilon>0$ траектории с энергией $H=-\varepsilon$, отслеживающие цепочки гомоклинических траекторий $\left\{\gamma_{\kappa}\right\}$, соответствуют траекториям ДЛС $\left\{\Phi_{\kappa}, S\right\}$ с лагранжианами $\Phi_{\kappa}, S$, определенными на открытых подмножествах сферы $\Sigma$. Эта система имеет $m-1$ степеней свободы. Чтобы применить теорему 5, удобно определить другую ДЛС с $2 m-2$ степенями свободы.

Можно предположить, что $X_{\kappa}^{ \pm} \subset U_{s_{\kappa}^{ \pm}}$. Определим дискретный лагранжиан $L_{\kappa}$ на $X_{\kappa}^{-} \times X_{\kappa}^{+} \times\left(U_{s_{\kappa}^{+}} \cap \Sigma\right)$ формулой

$$
L_{\kappa}(x, y, \varepsilon)=\Phi_{\kappa}(x, \varepsilon)+S\left(x_{+}, y_{-}, \varepsilon\right), \quad x=\left(x_{-}, x_{+}\right), \quad y=\left(y_{-}, y_{+}\right) .
$$

Этот лагранжиан имеет $2 m-2$ степени свободы.

Для заданного пути $\mathbf{k}=\left(\kappa_{i}\right)$ в графе $\Gamma_{-}$получим дискретный функционал действия

$$
A_{\mathbf{k}}(\mathbf{x})=\sum L_{\kappa_{i}}\left(x_{i}, x_{i+1}, \varepsilon\right), \quad x_{i}=\left(x_{i}^{-}, x_{i}^{+}\right) .
$$

Как отмечено в п. 3.4, траектории с энергией $H=-\varepsilon$, отслеживающие гомоклинические цепочки $\left(\gamma_{\kappa_{i}}\right)$, соответствуют критическим точкам функционала $A_{\mathbf{k}}$, т. е. траекториям ДЛС $\mathscr{L}=\left\{L_{\kappa}\right\}_{\kappa \in J}$. 
С помощью калибровочного преобразования заменим лагранжиан $L_{\kappa}$ на антиинтегрируемый лагранжиан

$$
\widehat{L}_{\kappa}(x, y, \varepsilon)=L_{\kappa}(x, y)+S_{-}\left(x_{-}\right)-S_{-}\left(y_{-}\right)=R_{\kappa}(x)+O\left(\varepsilon^{\alpha}\right),
$$

где функция $R_{\kappa}$ имеет невырожденную критическую точку $\left(q_{\kappa}^{-}, q_{\kappa}^{+}\right)$. Таким образом, теорема 10 вытекает из теоремы 5.

Теорема 10 сформулирована в [72] (в другой форме) и доказана в [24] (для положительно определенных лагранжевых систем) и в [71]. В [24] использовались вариационные методы, так что трансверсальность гомоклинической траектории не предполагалась. Аналогичные утверждения выполнены для систем с несколькими гиперболическими положениями равновесия на уровне энергии.

Рассмотрим, например, лагранжеву систему на компактном многообразии $M$ с лагранжианом $L(q, \dot{q})=\|\dot{q}\|^{2} / 2-V(q)$, где $V$ достигает максимума на множестве $A=\left\{a_{1}, \ldots, a_{n}\right\}$ и каждая точка максимума невырождена. Тогда существует много минимизирующих действие Мопертюи гетероклинических траекторий, соединяющих точки множества $A$, например, каждая пара точек соединяется цепочкой гетероклинических траекторий. Предположим, что минимальная гетероклиническая траектория $\gamma_{\kappa}$, соединяющая точки $a_{\kappa}^{-}$и $a_{\kappa}^{+}$, не принадлежит ни сильному устойчивому, ни сильному неустойчивому многообразию, и определим $s_{\kappa}^{ \pm}= \pm$как выше. Определим граф $\Gamma_{-}$, соединяя $\kappa$ и $\kappa^{\prime}$ ребром, если $a_{\kappa^{\prime}}=a_{\kappa}^{+}$и $s_{\kappa}^{+}=s_{\kappa^{\prime}}^{-}$. Тогда для малых $\varepsilon>0$ и любого пути на графе $\Gamma_{-}$существует траектория с энергией $-\varepsilon$, отслеживающая соответствующую цепочку гетероклинических траекторий. В [8] приведены интересные конкретные примеры.

6.2. Задача $n$ центров с малыми массами. Задача, рассматриваемая в данном пункте, во многом аналогична задаче о бильярде из п. 5.3: вместо малых рассеивателей имеются малые особенности потенциальной энергии.

Пусть $M$ - гладкое многообразие и $A=\left\{a_{1}, \ldots, a_{n}\right\}$ - конечное множество в $M$. Рассмотрим лагранжеву систему с конфигурационным пространством $M \backslash A$ и лагранжианом

$$
L(q, \dot{q}, \varepsilon)=L_{0}(q, \dot{q})-\varepsilon V(q)
$$

где $\varepsilon>0$ - малый параметр. Предположим, что функция $L_{0}$ гладкая на $T M$ и квадратичная по скорости:

$$
L_{0}(q, \dot{q})=\frac{1}{2}\|\dot{q}\|^{2}+\langle w(q), \dot{q}\rangle-W(q)
$$

где $\|\cdot\|$ - риманова метрика на $M$.

Пусть потенциальная энергия $V$ является гладкой функцией на $M \backslash A$ с ньютоновыми особенностями: в малом шаре $U_{k}$ с центром $a_{k}$

$$
V(q)=-\frac{\phi_{k}(q)}{\operatorname{dist}\left(q, a_{k}\right)},
$$


где $\phi_{k}$ - гладкая положительная функция на $U_{k}$. Расстояние задается римановой метрикой $\|\cdot\|$. Назовем систему с лагранжианом (6.2) задачей $n$ центров. При $\varepsilon=0$ предельная система с лагранжианом (6.3) не имеет особенностей.

Пусть

$$
H(q, \dot{q}, \varepsilon)=H_{0}+\varepsilon V, \quad H_{0}(q, \dot{q})=\frac{1}{2}\|\dot{q}\|^{2}+W(q)
$$

- интеграл энергии. Зафиксируем уровень энергии $\{H=E\}$ такой, что область $D=\{W<E\}$ содержит множество $A$.

Будем называть траекторию $\gamma:[a, b] \rightarrow D$ предельной системы с лагранжианом (6.3) невырожденной траекторией столкновения, если $\gamma(a), \gamma(b) \in A$, $\gamma(t) \notin A$ при $a<t<b$ и точки $\gamma(a), \gamma(b)$ не сопряжены вдоль $\gamma$ на уровне энергии $H=E$, т. е. для функционала действия Мопертюи.

Предположим, что существует несколько невырожденных траекторий столкновения $\left\{\gamma_{\kappa}\right\}_{\kappa \in J}$, соединяющих точки $a_{\kappa}^{-}, a_{\kappa}^{+} \in A$. Обозначим через $v_{\kappa}^{-}$и $v_{\kappa}^{+}$ начальную и конечную скорости траектории $\gamma_{\kappa}$. Рассмотрим граф $\Gamma$ с вершинами $J$, в котором вершины $\kappa, \kappa^{\prime}$ соединены ребром, если $a_{\kappa}^{+}=a_{\kappa^{\prime}}^{-}$и $v_{\kappa}^{+} \neq \pm v_{\kappa^{\prime}}^{-}$.

Следующее утверждение доказано в [19].

Tеорема 11. Существует $\varepsilon_{0}>0$ такое, что для всех $\varepsilon \in\left(0, \varepsilon_{0}\right]$ и любого пути $\kappa=\left(\kappa_{i}\right)_{i \in \mathbb{Z}}$ в графе $\Gamma$ существует единственная ( времени) гиперболическая траектория энергии $E$, отслеживающая иепочку $\left(\gamma_{\kappa_{i}}\right)_{i \in \mathbb{Z}}$ траекторий столкновения.

Таким образом, система имеет инвариантное подмножество на уровне энергии $\{H=E\}$, на котором она сопряжена надстройке над топологической цепью Маркова. Топологическая энтропия положительна, если граф Г имеет разветвленный подграф.

Условия теоремы 11 используют только свойства лагранжиана $L_{0}$ и множества $A$, так что они не зависят от вида потенциальной энергии $V$ при условии, что она имеет ньютоновы особенности на множестве $A$. Можно также добавить к $L$ гладкое $O(\varepsilon)$-малое возмущение.

СлЕДСТВИЕ 2. Предположим, что $M$ - замкнутое многообразие и

$$
E>\min _{q \in M}\left(\frac{1}{2}\|w(q)\|^{2}+W(q)\right) .
$$

Тогда для любого $n \geqslant 2$, почти любого набора точек $a_{1}, \ldots, a_{n} \in M$ и достаточно малого $\varepsilon>0$ существует хаотическое гиперболическое инвариантное множество траекторий с энергией $E$, близких к цепочкам траекторий столкновения.

Действительно, из сделанного предположения вытекает, что метрика Якоби

$$
d s_{E}=\sqrt{2(E-W(q))}\|d q\|+\langle w(q), d q\rangle
$$

является положительно определенной финслеровой метрикой на $M$. Согласно теории Морса, точки общего положения можно соединить бесконечным числом невырожденных геодезических, т. е. траекторий с энергией $E$. Таким образом, 
любые две точки в конечном множестве $A \subset M$ общего положения можно соединить бесконечным числом невырожденных траекторий столкновения с энергией $E$ так, чтобы другие точки множества $A$ не лежали на этих траекториях.

Если $n$ достаточно велико, то хаотические траектории существуют по чисто топологическим причинам (см., например, [21]), так что малость $\varepsilon$ не нужна.

ЗАмЕчАниЕ 9. Из теоремы 11 вытекает другое следствие для систем без малого параметра. Пусть

$$
L(q, \dot{q})=\frac{1}{2}\|\dot{q}\|^{2}-V(q) .
$$

Рассмотрим траектории с большой энергией $E=\varepsilon^{-1}$. После замены времени $s=\varepsilon^{-1 / 2} t$ получим лагранжиан вида (6.2), где $L_{0}=\|\dot{q}\|^{2} / 2$. Предельная система описывает движение по инерции. Таким образом, имеет место заключение теоремы 11. См. [53] для случая классической задачи $n$ центров.

Перейдем к доказательству теоремы 11. Рассмотрим сначала предельную систему при $\varepsilon=0$. Положим $\Sigma_{k}=\partial U_{k}$. Для любого $x \in \Sigma_{k}$ существуют единственная траектория $\gamma_{x}^{+}$с энергией $E$, соединяющая точки $x$ и $a_{k}$ в области $U_{k}$, и единственная траектория $\gamma_{x}^{-}$с энергией $E$, соединяющая $a_{k}$ и $x$. Положим

$$
S_{k}^{ \pm}(x)=\int_{\gamma_{x}^{ \pm}} p d q=\int_{\gamma_{x}^{ \pm}} d s_{E} .
$$

Тогда $S_{k}^{ \pm}$- гладкие функции на $\Sigma_{k}$.

Обозначим через $u^{+}(x)$ и $u^{-}(x)$ векторы скорости траекторий $\gamma_{x}^{ \pm}$в точке $a_{k}$. Зафиксируем малое $\delta>0$ и положим

$$
X_{k}=\left\{(x, y) \in \Sigma_{k}^{2}:\left\|u_{+}(x)-u_{-}(y)\right\| \geqslant \delta\right\} .
$$

ЛЕмма 6. Пусть $\varepsilon_{0}>0$ достаточно мало $u \varepsilon \in\left(0, \varepsilon_{0}\right]$.

1) Для любого $(x, y) \in X_{k}$ существует единственная траектория $\gamma=\gamma_{x, y}^{\varepsilon}$ с энергией $E$, соединяющая точки $x$ и у в области $U_{k}$.

2) Траектория $\gamma$ гладко зависит от $x, y$.

3) Действие Мопертюи

$$
S_{k}(x, y, \varepsilon)=\int_{\gamma} p d q
$$

- гладкая функиия на $X_{k} \times\left(0, \varepsilon_{0}\right]$ u, с точностью до константы $\varepsilon \log \varepsilon$,

$$
Q_{k}(x, y, \varepsilon)=S_{k}^{-}(x)+S_{k}^{+}(y)+\varepsilon u_{k}(x, y, \varepsilon),
$$

где функция $u_{k}$ равномерно $C^{2}$-ограничена на $X_{k}$ при $\varepsilon \rightarrow 0$.

Доказательство основано на регуляризации особенностей и на модифицированной лемме 3. Модификация требуется, поскольку все положительные собственные значения регуляризованной системы совпадают, что отличается от условий леммы 3 (см. [19]). 
Для любого $\kappa \in J$ обозначим через $x_{\kappa} \in \Sigma_{\kappa^{-}}$и $y_{\kappa} \in \Sigma_{\kappa^{+}}$точки пересечения траектории столкновения $\gamma_{\kappa}$ со сферами $\Sigma_{\kappa^{-}}$и $\Sigma_{\kappa^{+}}$соответственно. Если сферы $\Sigma_{k}$ достаточно малы, то точки $x_{\kappa}$ и $y_{\kappa}$ не сопряжены вдоль $\gamma_{\kappa}$.

Пусть $U_{\kappa}^{-} \subset \Sigma_{\kappa^{-}}$- малая окрестность точки $x_{\kappa}$, а $U_{\kappa}^{+} \subset \Sigma_{\kappa^{+}}$- малая окрестность точки $y_{\kappa}$. Если $\delta>0$ достаточно мало, то можно предполагать, что для любого ребра $\left(\kappa, \kappa^{\prime}\right)$ выполнено включение $U_{\kappa}^{+} \times U_{\kappa^{\prime}}^{-} \subset X_{k}$, где $a_{k}=a_{\kappa}^{+}=a_{\kappa^{\prime}}^{-}$. Если окрестности $U_{\kappa}^{ \pm}$достаточно малы и $\varepsilon \in\left(0, \varepsilon_{0}\right)$, то каждые две точки $x \in U_{\kappa}^{-}$и $y \in U_{\kappa}^{+}$соединены единственной траекторией $\beta_{\varepsilon}$ с энергией $H=E$, близкой к траектории $\gamma_{\kappa}$. Обозначим через

$$
\Phi_{\kappa}(x, y, \varepsilon)=\int_{\beta_{\varepsilon}} p d q
$$

действие Мопертюи траектории $\beta_{\varepsilon}$. Тогда $\Phi_{\kappa}-$ гладкая функция на $U_{\kappa}^{-} \times U_{\kappa}^{+}$.

ЛЕмма 7. Функция $R_{\kappa}(x, y)=\Phi_{\kappa}(x, y, 0)+S_{\kappa^{-}}^{-}(x)+S_{\kappa^{+}}^{+}(y)$ на $U_{\kappa}^{-} \times U_{\kappa}^{+}$ имеет невырожденную критическую точку $\left(x_{\kappa}, y_{\kappa}\right)$.

Эта лемма вытекает из предположения, что $\gamma_{\kappa}$ - невырожденная критическая точка функционала действия. В самом деле, $R_{k}(x, y)$ - действие Мопертюи кусочно гладкой траектории предельной системы, полученной склеиванием траекторий $\gamma_{x}^{-}, \beta_{0}$ и $\gamma_{y}^{+}$. Поэтому $R_{k}$ есть ограничение функционала действия на конечномерное подмногообразие, состоящее из ломаных траекторий (с точками излома $x, y)$, соединяющих $a_{\kappa^{-}}$и $a_{\kappa^{+}}$.

Определим дискретный лагранжиан системы с $2 m-2$ степенями свободы по формуле

$$
L_{\kappa}\left(z_{-}, z_{+}, \varepsilon\right)=\Phi_{\kappa}\left(z_{-}\right)+Q_{\kappa^{+}}\left(y_{-}, x_{+}, \varepsilon\right), \quad z_{-}=\left(x_{-}, y_{-}\right), \quad z_{+}=\left(x_{+}, y_{+}\right) .
$$

Для любого пути $\mathbf{k}=\left(\kappa_{i}\right)$ на графе Г критическая точка функционала

$$
A_{\mathbf{k}}(\mathbf{z})=\sum L_{\kappa_{i}}\left(z_{i}, z_{i+1}, \varepsilon\right), \quad z_{i} \in U_{\kappa_{i}}^{-} \times U_{\kappa_{i}}^{+},
$$

соответствует траектории с энергией $H=E$, которая отслеживает цепочку траекторий столкновения $\left(\gamma_{\kappa_{i}}\right)$.

Как и в п. 6.1, заменим $L_{\kappa}$ на калибровочно эквивалентный антиинтегрируемый лагранжиан

$$
\widehat{L}_{\kappa}\left(z_{-}, z_{+}, \varepsilon\right)=L\left(z_{-}, z_{+}, \varepsilon\right)+S_{\kappa^{-}}^{-}\left(x_{-}\right)-S_{\kappa^{+}}^{-}\left(x_{+}\right)=R_{\kappa}\left(z_{-}\right)+O(\varepsilon) .
$$

Согласно лемме 7 , функция $R_{\kappa}$ имеет невырожденную критическую точку. Таким образом, теорема 11 вытекает из теоремы 5 .

В качестве конкретного примера рассмотрим пространственную круговую ограниченную задачу трех тел (Солнце, Юпитер и астероид) в предположении, что масса $\varepsilon$ Юпитера много меньше массы $1-\varepsilon$ Солнца, причем Солнце и Юпитер движутся по круговым орбитам с угловой скоростью 1.

Рассмотрим движение астероида в системе отсчета Oxyz, вращающейся вокруг оси $z$, проходящей через Солнце $O=(0,0,0)$. Юпитер находится в точке 
$P=(1,0,0)$. Движение $q=(x, y, z)$ астероида описывается лагранжианом вида (6.2), где

$$
L_{0}(q, \dot{q})=\frac{1}{2}|\dot{q}|^{2}+x \dot{y}-y \dot{x}+\frac{1}{2}|q|^{2}+\frac{1}{|q|}, \quad V(q)=\frac{1}{|q|}-\frac{1}{|q-P|}+x .
$$

Имеем $M=\mathbb{R}^{3} \backslash\{O\}$, а множество особенностей состоит из одной точки $P$. Интеграл энергии во вращающейся системе отсчета,

$$
H=\frac{1}{2}|\dot{q}|^{2}-\frac{1}{2}|q|^{2}-\frac{1-\varepsilon}{|q|}-\frac{\varepsilon}{|q-p|}+\varepsilon x,
$$

называется интегралом Якоби, а $C=-2 H$ называется постоянной Якоби.

При $\varepsilon=0$ предельная система - это задача Кеплера Солнце-астероид. Ее ограниченными траекториями являются представленные во вращающейся системе отсчета эллипсы с параметрами $a, e, \iota$, где $a-$ большая полуось, $e-$ эксцентриситет, а $\iota$ - наклонение траектории к плоскости Оху. Частота обращения $\Omega$ равна $a^{-3 / 2}$, а постоянная Якоби $C$ равна $a^{-1}+2 \sqrt{a\left(1-e^{2}\right)} \cos \iota$. Траектории соударения - это дуги эллипсов с началом и концом в точке $P$.

При $C \in(-2,+3)$ определим множество $A_{C}$ допустимых частот:

- $(0,1)$, если $C \in[-1,+2]$,

- $\left(0,(2+C)^{3 / 2}\right)$, если $C \in(-2,-1)$,

- $\left((3-C)^{3 / 2}, 1\right)$, если $C \in(2,3)$.

Следующее утверждение доказано в [20].

Теорема 12. Для любого $C \in(-2,+3)$ множество $A_{C}$ имеет плотное подмножество $S$ такое, что для любого $\Omega \in S$ существует невырожденная траектория соударения $\gamma_{\Omega}$ с частотой $\Omega$ и наклонением $\iota=\cos ^{-1}\left(C / 2-\Omega^{2 / 3}\right)$.

Тогда из теоремы 11 вытекает следующее утверждение.

СЛЕДСТВИЕ 3. Для любого конечного множества $\Lambda \subset S$ существует $\varepsilon_{0}>0$ такое, что для любой последовательности $\left(\Omega_{n}\right)_{n \in \mathbb{Z}}$ в множестве $\Lambda$ и любого $\varepsilon \in\left(0, \varepsilon_{0}\right)$ существует траектория ограниченной задачи трех тел с постоянной Якоби $C$, которая $O(\varepsilon)$-отслеживает иепочку кеплеровых траекторий соударения $\gamma_{\Omega_{n}}$.

Траектории задачи трех тел, которые отслеживают цепочки траекторий задачи Кеплера, названы Пуанкаре решениями второго рода.

В [17] и [22] аналогичные утверждения получены для ограниченной эллиптической и неограниченной плоской задач трех тел с двумя малыми массами. Доказательство основано на редукции к ДЛС, но лагранжиан не совсем антиинтегрируемый. Требуемое обобщение теоремы 5 доказано в [16], [23].

6.3. Лагранжевы системы, медленно зависящие от времени. Самый прямой аналог антиинтегрируемой ДЛС - это непрерывная лагранжева система с лагранжианом вида

$$
L=L(q, \varepsilon \dot{q}, t, \varepsilon), \quad q \in M,
$$


где $\varepsilon>0$ - малый параметр. Например,

$$
L=\frac{\varepsilon^{2}}{2}\|\dot{q}\|^{2}+U(q, t)
$$

где $\|\cdot\|$ - риманова метрика на $M$, возможно, зависящая от времени $t$. Лагранжиан (6.9) напоминает антиинтегрируемый дискретный лагранжиан (5.2). Таким образом, можно ожидать, что предел $\varepsilon \rightarrow 0$ аналогичен антиинтегрируемому пределу в ДЛС.

Переходя к быстрому времени $s=t / \varepsilon$, получим систему, медленно зависящую от времени $s$ :

$$
L=L\left(q, q^{\prime}, t, \varepsilon\right), \quad q^{\prime}=\frac{d q}{d s} .
$$

Если $L$ удовлетворяет условию Лежандра, то система может быть представлена в гамильтоновой форме:

$$
q^{\prime}=\partial_{p} H, \quad p^{\prime}=-\partial_{q} H, \quad t^{\prime}=\varepsilon,
$$

где

$$
p=\partial_{q^{\prime}} L\left(q, q^{\prime}, t, \varepsilon\right), \quad H(q, p, t, \varepsilon)=\left\langle p, q^{\prime}\right\rangle-L .
$$

Полагая $z=(q, p)$, получим дифференциальное уравнение вида

$$
z^{\prime}=v(z, t, \varepsilon), \quad t^{\prime}=\varepsilon .
$$

Это стандартная форма сингулярно возмущенного дифференциального уравнения [73].

Далее будет изложена упрощенная версия одной из теорем работы [18]. Ссылки на предшествующие классические работы Черри и Палмера приведены в [18]. Методы, аналогичные антиинтегрируемому пределу, использовались в [61] при исследовании задачи Мезера. Интересные результаты о хаотическом возрастании энергии в системах вида (6.9) недавно были получены в [45].

При $\varepsilon=0$ лагранжиан (6.11) принимает вид

$$
L_{t}\left(q, q^{\prime}\right)=L\left(q, q^{\prime}, t, 0\right)
$$

где время $t$ заморожено. Поэтому энергия $H_{t}(q, p)=H(q, p, t, 0)$ - первый интеграл замороженной системы.

Предположим, что для каждого $t$ замороженная система имеет гиперболическое положение равновесия $O_{t}$ и существует гомоклиническая траектория $\gamma_{t}: \mathbb{R} \rightarrow M$ к положению равновесия: $\gamma_{t}( \pm \infty)=O_{t}$. Без ограничения общности предположим, что $H_{t}=0$ в положении равновесия $O_{t}$.

ЗАмЕчАниЕ 10. Для натуральной системы (6.10) на компактном многообразии гомоклиническая траектория всегда существует, если $O_{t}$ - точка минимума потенциала $U_{t}=U(q, t)$ (см. [14]). Если многообразие $M$ неодносвязно, то число таких траекторий не меньше наименьшего числа образующих полугруппы $\pi_{1}(M)$. 
Обозначим через $I$ открытое множество $t$ таких, что гомоклиника $\gamma_{t}$ трансверсальная: пересечение устойчивого и неустойчивого многообразий $W_{t}^{ \pm}$ вдоль $\gamma_{t}$ трансверсально на уровне энергии $\left\{H_{t}=0\right\}$. Действие Мопертюи

$$
f(t)=J\left(\gamma_{t}\right)=\int_{\gamma_{t}} p d q
$$

является гладкой функцией на $I$. Легко видеть, что

$$
f^{\prime}(t)=-\left.\int_{-\infty}^{\infty}\left(\partial_{t} H_{t}\right)\right|_{\gamma_{t}(s)} d s,
$$

где гомоклиническая траектория $\gamma_{t}$ параметризована быстрым временем $s$. Поэтому $f$ является аналогом функции Пуанкаре-Мельникова.

Для простоты предположим, что лагранжиан 1-периодичен по времени. Возьмем конечное множество невырожденных критических точек функции $f$ в $\mathbb{T}=\mathbb{R} / \mathbb{Z}$. Соответствующие точки в $\mathbb{R}$ образуют дискретное периодическое множество $K \subset \mathbb{R}$. Если $\rho>0$ достаточно мало, то интервалы $I_{k}=(k-\rho, k+\rho)$, $k \in K$, не пересекаются.

Теорема 13. Забиксируем малое $\delta>0$ и константы $0<c_{1}<c_{2}$. Предположим, что $\varepsilon>0$ достаточно мало. Тогда для любой возрастающей последовательности $k_{i} \in K$ существуют единственная траектория $q(t)$ и последовательность $t_{i} \in I_{k_{i}}, c_{1}<T_{i}<c_{2}$ такие, что

(a) $d\left(q(t), \gamma_{k_{i}}(\mathbb{R})\right) \leqslant \delta$ npu $t \in\left[t_{i}, t_{i}+\varepsilon T_{i}\right]$

(b) $d\left(q(t), O_{t}\right) \leqslant \delta$ npu $t \in\left[t_{i}+\varepsilon T_{i}, t_{i+1}\right]$

u, более того,

(c) $\left|t_{i}-k_{i}\right| \leqslant C \varepsilon u d\left(q(t), \gamma_{k_{i}}(\mathbb{R})\right) \leqslant C \varepsilon$ npu $t \in\left[t_{i}, t_{i}+\varepsilon T_{i}\right]$.

Назовем $q(t)$ пульсирующей траекторией (см. рис. 6). Утверждение теоремы 13 справедливо также для непериодических $L$, если выполнены некоторые предположения равномерности.

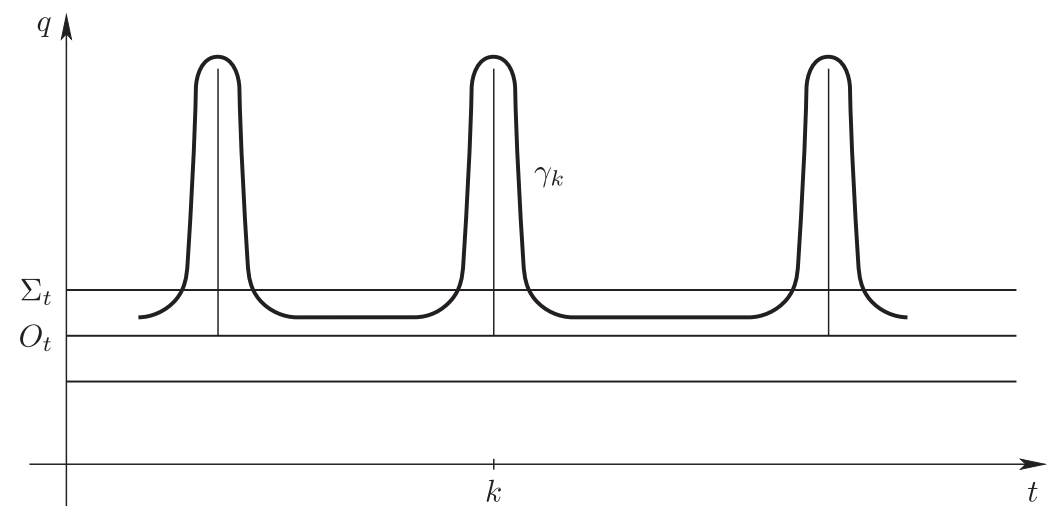

Рис. 6 
В работе [18] аналогичное утверждение доказано без предположения трансверсальности. Рассмотрим систему (6.10) на неодносвязном компактном многообразии $M$, и пусть $f(t)$ - минимум действия нестягиваемых гомоклиник для замороженной системы. Тогда достаточно предположить, что $f$ непостоянна.

ДокАЗАТЕЛЬСтво теОРемы 13. Пусть сначала $\varepsilon=0$. Поскольку $O_{t}-$ гиперболическое положение равновесия предельной системы, оно имеет локальные устойчивое и неустойчивое лагранжевы многообразия $W_{t}^{+}$и $W_{t}^{-}$в фазовом пространстве $T^{*} M$. Предположим для простоты, что проекция $W_{t}^{ \pm} \rightarrow M$ невырождена в точке $O_{t}$. Это всегда так, если замороженная система натуральная, как в (6.10); в общем случае этого можно добиться, пошевелив симплектические координаты около $O_{t}$. Поэтому

$$
W_{t}^{+}=\left\{(q, p): q \in D_{t}, p=-\nabla S_{t}^{+}(q)\right\}, \quad W_{t}^{-}=\left\{(q, p): q \in D_{t}, p=\nabla S_{t}^{-}(q)\right\},
$$

где $D_{t}$ - малая $\delta$-окрестность точки $O_{t}$. Поскольку производящие функции $S_{t}^{ \pm}$ определены с точностью до аддитивной функции времени, без ограничения общности предположим, что $S_{t}^{ \pm}\left(O_{t}\right)=0$. Тогда $S_{t}^{+}(q)$ - действие Мопертюи траектории замороженной системы, начинающейся в точке $q$ и асимптотической к точке $O_{t}$ в быстром времени $s \rightarrow+\infty$, а $S_{t}^{-}-$действие траектории, асимптотической к $O_{t}$ при $s \rightarrow-\infty$ и заканчивающейся в точке $q$.

В следующей лемме используется медленное время $t$.

Лемма 8. Зафиксируем $0<c_{1}<c_{2}$, и пусть $\varepsilon>0$ достаточно мало. Для любых $a<b$ таких, что $b-a \in\left(c_{1}, c_{2}\right), u$ любых $x \in D_{a}, y \in D_{b}$ существует единственная траектория $q(t) \in D_{t}, a \leqslant t \leqslant b$, такая, что $q(a)=x u q(b)=y$. Более того, справедливы следуюшие утверждения:

1) функция $q(t)=q(t, a, b, x, y, \varepsilon)$ гладкая при $\varepsilon>0$;

2) траектория $q(t)$ является $C^{0}$-близкой к склейке асимптотических траекторий замороженной системы;

3) если через

$$
S(a, b, x, y, \varepsilon)=\varepsilon^{-1} \int_{a}^{b} L(q(t), \varepsilon \dot{q}(t), t, \varepsilon) d t
$$

обозначить действие траектории $q(t)$, нормализованное $\kappa$ быстрому времени, mo

$$
S=S^{+}(x)+S^{-}(y)+\varepsilon v(a, b, x, y, \varepsilon),
$$

где $v$ - гладкая функиия при $\varepsilon>0 u\|v\|_{C^{2}} \leqslant C$, где константа $C$ не зависит om $\varepsilon$.

Отметим, что $q(t)$ имеет нерегулярное поведение при $\varepsilon \rightarrow 0$ (ее производная по времени неограничена порядка $\left.\varepsilon^{-1}\right)$, в то же время производящая функция $S$ регулярна при $\varepsilon \rightarrow 0$. Если лагранжиан $(6.9)$ не зависит от $t$ и $\varepsilon$, то лемма 8 вытекает из леммы Шильникова [63] или сильной $\lambda$-леммы [39].

В общем случае существование решения граничной задачи можно вывести из результатов теории сингулярно возмущенных дифференциальных уравнений [73]. 
Пусть $p(t)=\partial_{q^{\prime}} L(q(t), \varepsilon \dot{q}(t), t, \varepsilon)$ - импульс (6.13) траектории $q(t)$. По формуле первой вариации

$$
\begin{aligned}
\partial_{x} S & =-p(a), & \partial_{y} S & =p(b), \\
\partial_{a} S & =H(x, p(a), a, \varepsilon), & \partial_{b} S & =-H(y, p(b), b, \varepsilon) .
\end{aligned}
$$

Лемма 8 описывает траектории, которые находятся около положения равновесия $O_{t}$ в течение конечного интервала медленного времени $t$. Теперь будут описаны траектории, отслеживающие гомоклиническую траекторию $\gamma_{k}$ в интервале медленного времени порядка $\varepsilon^{-1}$. Поэтому будет использовано быстрое время $s=\varepsilon^{-1} t$.

Для упрощения обозначений предположим, что окрестность $D_{t}=D_{k}$ не зависит от $t$ при $t \in I_{k}$. Положим $\Sigma_{k}=\partial D_{k}$. Пусть $\gamma_{k}(0), \gamma_{k}\left(\tau_{k}\right)-$ точки пересечения гомоклиники $\gamma_{k}: \mathbb{R} \rightarrow M$ со сферой $\Sigma_{k}$. Пошевелив $\delta$, если требуется, предположим, что эти точки не сопряжены вдоль $\gamma_{k}$. Тогда при $z=(x, y, t, T)$, близких к точке $z_{k}^{0}=\left(\gamma_{k}(0), \gamma_{k}\left(\tau_{k}\right), k, \tau_{k}\right)$ и достаточно малом $\varepsilon>0$ существует траектория $\beta_{\varepsilon}(s), 0 \leqslant s \leqslant T$, системы с лагранжианом $L\left(q, q^{\prime}, t+\varepsilon s, \varepsilon\right)$, удовлетворяющая граничным условиям $\beta_{\varepsilon}(0)=x, \beta_{\varepsilon}(T)=y$, и близкая к гомоклинической траектории $\gamma_{k}$. Пусть

$$
\Phi_{k}(z, \varepsilon)=\int_{0}^{T} L\left(\beta_{\varepsilon}(s), \beta_{\varepsilon}^{\prime}(s), t+\varepsilon s, \varepsilon\right) d s
$$

- ее действие. Производные функции $\Phi_{k}$ удовлетворяют равенствам, аналогичным равенствам (6.17):

$$
\begin{aligned}
\partial_{x} \Phi_{k} & =-p(0), & \partial_{y} F_{k} & =p(T), \\
\partial_{t} \Phi_{k} & =H(x, p(0), t, \varepsilon), & \partial_{T} \Phi_{k} & =-H(y, p(T), t+\varepsilon T, \varepsilon),
\end{aligned}
$$

где $p(s)$ - импульс траектории $\beta_{\varepsilon}(s)$.

ЛЕмма 9. Функиця

$$
R_{k}(z)=S_{t}^{-}(x)+\Phi_{k}(x, y, t, T, 0)+S_{t}^{+}(y), \quad x, y \in \Sigma_{k},
$$

имеет невырожденную критическую точку $z_{k}^{0}$.

Действительно, $R_{k}(z)$ - действие для склейки асимптотической траектории замороженной системы, начинающейся в точке $O_{t}$, заканчивающейся в точке $x$, траектории $\beta_{0}$, соединяющей точки $x$ и $y$ в окрестности траектории $\gamma_{k}$, и траектории, начинающейся в точке $y$ и асимптотической к равновесию $O_{t}$. Уравнение $\partial_{T} R_{k}=0$ влечет $H_{t}=0$ вдоль $q(s)$. Тогда из равенств $\partial_{x} R_{k}=0$ и $\partial_{y} R_{k}=0$ вытекает, что эта склейка - гладкая траектория замороженной системы, гомоклиническая к равновесию $O_{t}$. Таким образом, эта траектория совпадает с $\gamma_{t}$. Наконец, уравнение $\partial_{t} R_{k}=0$ означает, что $t-$ критическая точка функции $f(t)$.

Определим ДЛС, описывающую пульсирующие траектории. Пусть $J=\{\kappa=$ $\left.\left(\kappa_{-}, \kappa_{+}\right) \in K^{2}: \kappa_{-}<\kappa_{+}\right\}-$множество вершин графа. Соединим вершины 
$\kappa, \kappa^{\prime} \in J$ ребром, если $\kappa_{+}=\kappa_{-}^{\prime}$. Определим дискретный лагранжиан системы с $2 m$ степенями свободы по формуле

$$
L_{\kappa}\left(z_{-}, z_{+}, \varepsilon\right)=\Phi_{k}\left(z_{-}, \varepsilon\right)+S\left(t_{-}+\varepsilon T_{-}, t_{+}, y_{-}, x_{+}, \varepsilon\right), \quad z_{ \pm}=\left(x_{ \pm}, y_{ \pm}, t_{ \pm}, T_{ \pm}\right) .
$$

Таким образом, $L_{\kappa}\left(z_{-}, z_{+}, \varepsilon\right)$ - действие ломаной траектории, которая начинается в точке $x_{-} \in \Sigma_{\kappa_{-}}$в момент времени $t_{-}$, отслеживает гомоклиническую траекторию $\gamma_{\kappa_{-}}$, пока не пересечет $\Sigma_{\kappa_{+}}$в точке $y_{-}$в момент времени $t+\varepsilon T_{-}$, движется вблизи гиперболического положения равновесия $O_{t}$ при $t_{-}+\varepsilon T_{-} \leqslant t \leqslant t_{+}$и заканчивается в точке $x_{+} \in \Sigma_{\kappa_{+}}$в момент времени $t_{+}$.

Из равенств (6.17), (6.18) вытекает, что критические точки функционала

$$
A_{\mathbf{k}}(z)=\sum L_{\kappa_{i}}\left(z_{i}, z_{i+1}, \varepsilon\right), \quad \kappa_{i}=\left(k_{i}, k_{i+1}\right),
$$

где

$$
z_{i}=\left(x_{i}, y_{i}, t_{i}, T_{i}\right), \quad x_{i}, y_{i} \in \Sigma_{k_{i}}, \quad t_{i} \in I_{k_{i}}, \quad T_{i}>0,
$$

соответствуют траекториям, отслеживающим цепочки гомоклиник $\left(\gamma_{k_{i}}\right)$.

Сделаем калибровочное преобразование, заменяющее $L_{\kappa}$ на лагранжиан

$$
\widehat{L}_{\kappa}\left(z_{-}, z_{+}, \varepsilon\right)=L_{\kappa}\left(z_{-}, z_{+}, \varepsilon\right)+S_{\kappa^{-}}\left(x_{-}\right)-S_{\kappa^{+}}\left(x_{+}\right)=R_{\kappa^{-}}\left(z_{-}\right)+O(\varepsilon),
$$

где $R_{\kappa^{-}}$имеет невырожденную критическую точку $z_{\kappa^{-}}^{0}$. Лагранжиан $\widehat{L}_{\kappa}$ имеет антиинтегрируемую форму.

Отметим, что граф Г, описывающий антиинтегрируемую систему, бесконечен. Однако лагранжиан инвариантен по отношению $\mathrm{k} \mathbb{Z}$-действию на $\mathbb{R}$, так что условия теоремы 6 выполнены. Теорема 13 доказана.

\section{7. Сепаратрисное отображение}

\section{1. Антиинтегрируемый предел в сепаратрисном отображении За-} славского. Рассмотрим интегрируемое сохраняющее площадь отображение $F_{0}$, имеющее гиперболическую неподвижную точку с двумя гомоклиническими петлями сепаратрис. Пусть $F_{\varepsilon}$ - возмущенное отображение, также сохраняющее площадь. В $\varepsilon$-окрестности невозмущенных петель сепаратрис динамика отображения $F_{\varepsilon}$ определяется сепаратрисным отображением.

Конструкция представлена на рис. 7. В левой части рисунка изображено фазовое пространство отображения $F_{\varepsilon}$. Видна гиперболическая неподвижная точка $p_{\varepsilon}$ и ее асимптотические кривые (сепаратрисы), расщепленные при $\varepsilon \neq 0$. Также имеются две серые области $\Delta_{\varepsilon}^{ \pm}$, на которых будет определено сепаратрисное отображение. Границами этих областей служат криволинейные четырехугольники. Их “горизонтальные" стороны могут считаться лежащими на инвариантных КАМ-кривых (это удобно, но необязательно), а "вертикальные стороны" в каждом четырехугольнике - образы друг друга при действии отображений $F_{\varepsilon}$ и $F_{\varepsilon}^{-1}$. Для любой точки $z \in \Delta_{\varepsilon}=\Delta_{\varepsilon}^{+} \cup \Delta_{\varepsilon}^{-}$ее образ $F_{\varepsilon}(z)$ лежит вне $\Delta_{\varepsilon}$. По определению образом точки $z$ при сепаратрисном отображении является $F_{\varepsilon}^{n}(z)$, где $n=n(\varepsilon)$ - минимальное натуральное число такое, что $F_{\varepsilon}^{n}(z) \in \Delta_{\varepsilon}$. На рис. 7 показаны две точки $z$ и их образы. 


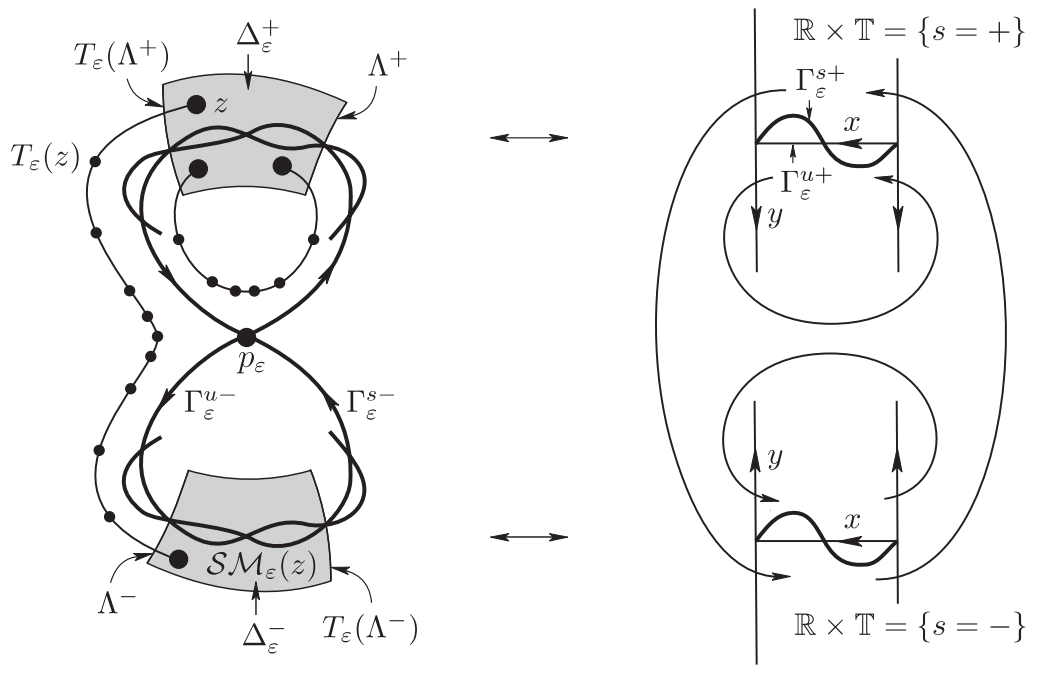

Рис. 7. Сепаратрисное отображение

В некоторых удобных координатах сепаратрисное отображение можно вычислить в виде "явная часть + малые поправки":

$$
\begin{aligned}
\left.\left(\begin{array}{l}
y \\
x \\
\sigma
\end{array}\right) \mapsto\left(\begin{array}{c}
y_{+} \\
x_{+} \\
\sigma_{+}
\end{array}\right), \quad \begin{array}{l}
y_{+} \\
\end{array}\right) \quad x_{+}=x+\frac{\partial V_{\sigma}}{\partial x}+O(\varepsilon), \\
\sigma_{+}=\sigma \operatorname{sign} y_{+}, \quad \sigma, \sigma_{+} \in\{-1,1\} .
\end{aligned}
$$

Здесь переменная $x \bmod 1$ изменяется вдоль сепаратрисы, переменная $y$ - поперек сепаратрисы, а дискретная переменная $\sigma= \pm 1$ указывает, возле какой из петель орбита находится в данный момент времени. Функции $V_{\sigma}(x)$ (потенциалы Пуанкаре-Мельникова) периодичны с периодом 1. (Подробности можно найти, например, в [62], [70].)

Отображение (7.1) имеет вид

$$
y=\frac{\partial W}{\partial x}, \quad x_{+}=\frac{\partial W}{\partial y_{+}}, \quad \sigma_{+}=\sigma \operatorname{sign} y_{+}
$$

с производящей функцией

$$
W=W\left(y_{+}, x, \sigma\right)=x y_{+}-\lambda V_{\sigma}(x)+\frac{1+O(\varepsilon)}{\lambda}\left(\omega_{\sigma}+\log \left|y_{+}\right|-1\right) y_{+} .
$$

Первые два равенства в (7.2) можно записать в виде

$$
x_{+} d y_{+}+y d x=d W\left(y_{+}, x, \sigma\right) .
$$

Чтобы представить (7.1) в лагранжевой форме, рассмотрим другую производящую функцию (преобразование Лежандра $W$ )

$$
\lambda L=x_{+} y_{+}-W, \quad y_{+} d x_{+}-y d x=\lambda d L .
$$


Здесь мы используем тот факт, что лагранжиан определен с точностью до ненулевого постоянного множителя. Легко получить явный вид для $L$ :

$$
\begin{gathered}
L\left(x, x_{+}, \sigma, \vartheta_{+}\right)=(1+O(\varepsilon)) \vartheta_{+} e^{\lambda\left(x_{+}-x-\hat{\omega}_{\sigma}\right)}+V_{\sigma}(x), \\
\vartheta_{+}=\operatorname{sign} y_{+}, \quad \widehat{\omega}_{\sigma}=\omega_{\sigma}+\lambda^{-1} \log \lambda^{2} .
\end{gathered}
$$

Таким образом, сепаратрисное отображение - $\mathbb{Z}$-эквивариантная ДЛС, где $\mathbb{Z}$ действует на $M=\mathbb{R} \times \mathbb{Z}_{2} \times \mathbb{Z}_{2}$ сдвигами

$$
M \ni(x, \sigma, \vartheta) \mapsto k(x, \sigma, \vartheta)=(x+k, \sigma, \vartheta) .
$$

Лагранжев вид сепаратрисного отображения следующий:

$$
\left(\begin{array}{c}
x_{-} \\
x \\
\sigma_{-} \\
\vartheta
\end{array}\right) \mapsto\left(\begin{array}{c}
x \\
x_{+} \\
\sigma \\
\vartheta_{+}
\end{array}\right), \quad \begin{gathered}
\sigma=\sigma_{-} \vartheta \\
\frac{\partial}{\partial x}\left(L_{-}+L\right)=0
\end{gathered}
$$

где

$$
L_{-}=L\left(x_{-}, x, \sigma_{-}, \vartheta\right), \quad L=L\left(x, x_{+}, \sigma, \vartheta_{+}\right) .
$$

Первые два равенства в (7.5) возникают из определения переменной $\vartheta(\vartheta=$ $\operatorname{sign} I$ ) и из последнего равенства в (7.1). Третье равенство в (7.5) следует из (7.3), поскольку согласно (7.3) и аналогичному равенству $y d x-y_{-} d x_{-}=$ $d L_{-}$имеем

$$
y=-\frac{\partial L}{\partial x}=\frac{\partial L_{-}}{\partial x} .
$$

Легко проверить, что величины $x_{+}, \sigma$ и $\vartheta_{+}$выражаются единственным образом из (7.5) через $x_{-}, x, \sigma_{-}$и $\vartheta$.

Получаем ДЛС с добавочными дискретными переменными $\sigma$ и $\vartheta$. Любая последовательность

$$
\mathbf{z}=\left\{z_{j}\right\}, \quad z_{j}=\left(\begin{array}{c}
x_{j} \\
\sigma_{j} \\
\vartheta_{j}
\end{array}\right), \quad \sigma_{j+1}=\sigma_{j} \vartheta_{j+1},
$$

называется путем. Пусть $\Sigma$ - множество всех путей.

Вообще говоря, индекс $j$ принимает все целые значения. Однако можно рассматривать также полубесконечные и конечные пути. Пути, конечные слева, начинаются с тройки $z_{j}$, где $x_{j}=+\infty$. Пути, конечные справа, заканчиваются в $z_{j}$, где $x_{j}=-\infty$. Пути, конечные слева и справа, называются конечными.

Действие $A$ определено как конечная сумма

$$
A=A(\mathbf{z})=\sum_{j} L\left(x_{j}, x_{j+1}, \sigma_{j}, \vartheta_{j+1}\right) .
$$

Путь $\mathbf{z}^{0}$ называется экстремалью (или траекторией), если $\partial A /\left.\partial x_{j}\right|_{\mathbf{z}=\mathbf{z}^{0}}=0$ при вcex $j$. 
Отметим, что полуконечные траектории лежат на сепаратрисах. Конечные лежат как на устойчивой, так и на неустойчивой сепаратрисе, и, следовательно, являются гомоклиническими траекториями.

Определим расстояние $\rho$ на $\Sigma$ следующим образом. Пусть $\mathbf{z}^{\prime}$ и $\mathbf{z}^{\prime \prime}$ - пути, где

$$
z_{j}^{\prime}=\left(\begin{array}{c}
x_{j}^{\prime} \\
\sigma_{j}^{\prime} \\
\vartheta_{j}^{\prime}
\end{array}\right), \quad z_{j}^{\prime \prime}=\left(\begin{array}{c}
x_{j}^{\prime \prime} \\
\sigma_{j}^{\prime \prime} \\
\vartheta_{j}^{\prime \prime}
\end{array}\right) .
$$

Положим $\rho\left(\mathbf{z}^{\prime}, \mathbf{z}^{\prime \prime}\right)=\infty$, если последовательности $\sigma_{j}^{\prime}, \vartheta_{j}^{\prime}$ не совпадают с $\sigma_{j}^{\prime \prime}, \vartheta_{j}^{\prime \prime}$ или если для некоторого $j$ лишь одна из троек определена. В противном случае положим

$$
\rho\left(\mathbf{z}^{\prime}, \mathbf{z}^{\prime \prime}\right)=\sup _{j}\left|x_{j}^{\prime}-x_{j}^{\prime \prime}\right| .
$$

Здесь мы считаем, что $|-\infty-(-\infty)|=|+\infty-(+\infty)|=0$.

Пусть $\operatorname{Cr}(\sigma)$ - конечное множество невырожденных критических точек функции $V_{\sigma}$. Множество $\Sigma$ содержит подмножество П простых путей (кодов). По определению путь $\mathbf{z}$ простой, если $x_{j} \in \operatorname{Cr}\left(\sigma_{j}\right)$ при всех $j$.

Теорема 14. Предположим, что постоянные $c_{1} u c_{2}=c_{2}\left(c_{1}\right)$ достаточно велики. Тогда для любого простого пути $\mathbf{z}^{*}$ такого, что $x_{j}^{*}-x_{j+1}^{*}>c_{1} n р и$ всех $j$, существует единственная траектория $\widetilde{\mathbf{z}}$ в $c_{2}^{-1}$-окрестности пути $\mathbf{z}^{*}$. Траектория $\widetilde{\mathbf{z}}$ гиперболична.

Теорема 14 устанавливает символическую динамику в окрестности сепаратрис отображения, сохраняющего площадь. Теорему 14 можно вывести из теоремы 5 путем рассмотрения ДЛС с лагранжианом

$$
L_{\kappa}\left(x_{-}, x_{+}\right)=L\left(x_{-}+k, x_{+}, \sigma, \theta\right), \quad \kappa=(k, \sigma, \theta), \quad x_{ \pm} \in(0,2 \pi),
$$

где $k>c_{1}$ достаточно велика. Соответствующий граф имеет вершины $\kappa$. Вершины $\kappa, \kappa^{\prime}$ соединены ребром, если $\sigma^{\prime}=\sigma \theta$. Имеется ребро $\gamma$ для любой невырожденной критической точки $V_{\sigma}$ в $(0,2 \pi)$. Для больших $k$ ДЛС антиинтегрируема. Граф Г бесконечен, но условие U равномерной антиинтегрируемости очевидно выполнено.

Традиционный подход к символической динамике около сепаратрис представлен в [13], [2], [60]. Другой вариант сепаратрисного отображения построен В. С. Шильниковым и Л. П. Афраймовичем [1] (см. также [64]). Эта конструкция также приведена в п. 6.1.

7.2. Сепаратрисное отображение и диффузия Арнольда. Идеи антиинтегрируемого предела могут быть применены при исследовании проблемы диффузии Арнольда. Здесь мы обсудим только априори неустойчивый случай, в котором, в отличие от исходной (априори устойчивой) ситуации [3], невозмущенная интегрируемая система содержит нормально гиперболическое многообразие $N$. В возмущенной системе хаос в основном сконцентрирован около $N$ и его асимптотических многообразий. 
Мы рассматриваем неавтономную гамильтонову систему, близкую к интегрируемой на фазовом пространстве $\mathbb{T}_{x}^{n} \times \overline{\mathscr{D}} \times D \times \mathbb{T}_{t}$, где $\mathscr{D} \subset \mathbb{R}_{y}^{n}$ - открытая область с компактным замыканием $\overline{\mathscr{D}}$, а $D \subset \mathbb{R}_{(v, u)}^{2}$ - область. Функция Гамильтона и симплектическая структура следующие:

$$
\begin{gathered}
H(y, x, v, u, t, \varepsilon)=H_{0}(y, v, u)+\varepsilon H_{1}(y, x, v, u, t)+\varepsilon^{2} H_{2}(y, x, v, u, t, \varepsilon), \\
\omega=d y \wedge d x+d v \wedge d u .
\end{gathered}
$$

Как обычно, $\varepsilon \geqslant 0$ - малый параметр. Уравнения Гамильтона имеют вид

$$
\dot{y}=-\frac{\partial H}{\partial x}, \quad \dot{x}=\frac{\partial H}{\partial y}, \quad \dot{v}=-\frac{\partial H}{\partial u}, \quad \dot{u}=\frac{\partial H}{\partial v} .
$$

Предположим, что в невозмущенном гамильтониане переменные $у$ отделены от $u$ и $v$, т. е. $H_{0}(y, v, u)=F(y, f(v, u))$. Функция $f$ имеет невырожденную седловую точку $(v, u)=(0,0)$, являющуюся единственной критической точкой на компактной компоненте связности множества

$$
\gamma=\{(v, u) \in D: f(v, u)=f(0,0)\}
$$

В динамической терминологии $(0,0)$ - гиперболическое положение равновесия гамильтоновой системы $(D, d v \wedge d u, f)$ с одной степенью свободы, и соответствующие сепаратрисы сдвоены. Топологически эти сепаратрисы образуют восьмерку: две петли $\gamma^{ \pm}$, выходящие из одной точки, $\gamma=\gamma^{+} \cup \gamma^{-}$.

Таким образом, невозмущенное нормально гиперболическое многообразие есть

$$
N=\mathbb{T}_{x}^{n} \times \overline{\mathscr{D}} \times(0,0) \times \mathbb{T}_{t}
$$

Оно расслоено на торы

$$
\left.N_{y}=\{x, y, u, v, t): u=v=0, y=\mathrm{const}\right\}
$$

несущие квазипериодическую динамику с частотами

$$
\left(\begin{array}{c}
\nu(y) \\
1
\end{array}\right), \quad \nu(y)=\frac{\partial H_{0}}{\partial y}(y, 0,0) .
$$

Мы интересуемся возмущенной динамикой около многообразий, асимптотических к $N$.

Проблема диффузии Арнольда в априори неустойчивом случае имеет три аспекта, содержащиеся в следующей гипотезе.

ГиПотезА 1 [5], [69].

А (типичность). Диффузия существует для открытого плотного множества $C^{r}$-возмущений, где $r \in \mathbb{N} \cup\{\infty, \omega\}$ достаточно велико.

В (свобода). Проекиия дифбузионной траектории на у-пространство может двигатъся в малой окрестности произвольной гладкой кривой $\chi \subset \mathscr{D}$.

С (скорость). Имеются “быстрые" диффузионные траектории, у которых средняя скорость вдоль ұ - порядка $\varepsilon /|\log \varepsilon|$. 
Имеется несколько подходов к задаче. Традиционный подход основан на конструкции переходных цепочек гиперболических торов [3], [34], [35], [43], [36]-[38], позже он был эффективно дополнен идеями отображения рассеяния [36]-[38] и символической динамики в полисистемах [26]. Вариационный подход развивался в [9]-[11], [32], [33], [46], [49], [50].

Гипотеза 1 доказана только в случае $n=1$ (две с половиной степени свободы) [68]. Далее мы коротко изложим идеи и методы статьи [68]. Система $\left.(7.6)\right|_{\varepsilon=0}$ имеет $n$-параметрическое семейство (частично) гиперболических $(n+1)$-мерных торов $N_{y}$, расслаивающих нормально гиперболическое многообразие $N$. Многообразия, асимптотические к $N$, имеют две компоненты $\{y\} \times \mathbb{T}_{x}^{n} \times \gamma^{ \pm} \times \mathbb{T}_{t}$. Таким образом, при возмущении ситуация напоминает рассмотренную в п.7.1. В фазовом пространстве отображения за время 1 появляется картина, аналогичная представленной на рис. 7. Но, в отличие от случая, рассмотренного в п.7.1, теперь приходится иметь дело с семейством гиперболических торов и их асимптотических поверхностей. Сепаратрисное отображение по-прежнему можно получить [66], и явные формулы для него можно считать многомерным обобщением формул (7.1).

Сепаратрисное отображение можно представить в виде

$$
\begin{gathered}
\left(\zeta, \rho, \tau_{-}, \tau, \sigma_{-}, \theta\right) \mapsto\left(\zeta_{+}, \rho_{+}, \tau, \tau_{+}, \sigma, \theta_{+}\right), \\
\rho=\frac{\partial \mathscr{R}}{\partial \zeta}, \quad \zeta_{+}=\frac{\partial \mathscr{R}}{\partial \rho_{+}}, \quad \frac{\partial}{\partial \tau}\left(\mathscr{R}_{-}+\mathscr{R}\right)=0, \quad \sigma=\sigma_{-} \vartheta, \quad \vartheta_{+}=\sigma \sigma_{+}, \\
\mathscr{R}=R\left(\zeta, \rho_{+}, \tau, \tau_{+}, \sigma, \theta_{+}, t_{+}, \varepsilon\right), \quad \mathscr{R}_{-}=R\left(\zeta_{-}, \rho, \tau_{-}, \tau, \sigma_{-}, \theta, t, \varepsilon\right)
\end{gathered}
$$

(ср. с (7.5)). Здесь с точностью до малых поправок выполнено равенство $\varepsilon \rho=y$ и разность $\zeta-x-$ функция переменных $y, u, v$. Целочисленная переменная $t$ имеет смысл времени, в течение которого траектория отображения за время 1 движется вне аналогов областей $\Delta_{\varepsilon}^{ \pm}$(см. рис. 7$)$. Переменные $\sigma$ и $\vartheta$ аналогичны соответствующим переменным из п. 7.1, и

$$
\begin{gathered}
\mathscr{R}=\left\langle\rho_{+}, \zeta+\nu t_{+}\right\rangle-\left(\tau_{+}-\tau-t_{+}\right) \mathbf{H}\left(\varepsilon \rho_{+}, \zeta\right) \\
\quad-\vartheta_{+} e^{\lambda\left(\tau_{+}-\tau-\omega_{+}^{\sigma}\right)}+\widehat{\Theta}^{\sigma}\left(\varepsilon \rho_{+}, \zeta, \tau\right), \\
\omega_{+}^{\sigma}=t_{+}+\lambda^{-1} \log \varepsilon+f\left(\varepsilon \rho_{+}\right), \quad \nu=\nu\left(\varepsilon \rho_{+}\right), \quad \lambda=\lambda\left(\varepsilon \rho_{+}\right) .
\end{gathered}
$$

Если $n=0$, то переменные $\rho$ и $\zeta$ отсутствуют и в $\mathscr{R}$ остаются лишь последние два слагаемых. В этом случае с точностью до постоянного множителя $\mathscr{R}$ превращается в $L$ (см. (7.4)).

Нас интересует случай, когда величины $t_{+}+\lambda^{-1} \log \varepsilon$ превосходят большую положительную постоянную $K_{0}$, так что $e^{K_{0}}$ играет роль большого параметра в антиинтегрируемом пределе. Функция $\mathbf{H}$ является существенной только в малых окрестностях сильных (низкого порядка) резонансов

$$
\left\{\rho:\langle\nu(\rho), k\rangle+k_{0}=0\right\}, \quad k \in \mathbb{Z}^{n}, \quad k_{0} \in \mathbb{Z}, \quad|k|+\left|k_{0}\right|<C .
$$


Мы видим, что дискретная динамическая система (7.7) частично гамильтонова (относительно $\rho, \zeta$ ) и частично лагранжева (относительно $\tau$ ). Из-за присутствия новых гамильтоновых переменных приходится использовать некоторое обобщение метода антиинтегрируемого предела. К сожалению, в этом обобщении символическая динамика не настолько прозрачна и стандартна, как она выглядит в п. 4.1 или п. 7.1. Конструкция здесь следующая [67]. Имея конечный кусок траектории сепаратрисного отображения и соответствующую квазитраекторию - кусок той же длины (код), мы указываем правило, в соответствии с которым можно удлинить код, добавив новую точку. Затем согласно основному результату из [67] траекторию можно слегка продеформировать и продолжить так, чтобы она оставалась близкой к удлиненному коду. Итак, снова получаем пару: кусок траектории с кодом. Используя определенную свободу в правилах удлинения кода, можно пытаться толкать траекторию в желаемом направлении на $y$-пространстве.

Здесь возникает другая трудность. Возможность толкать орбиту в нужном направлении реализуется относительно просто в нерезонансной зоне, т. е. там, где вектор частот $(\nu(y), 1) \in \mathbb{R}^{n+1}(1$ - частота по времени) не допускает резонансов низкого порядка. Около резонанса конструкция хорошего удлинения кода становится отдельной деликатной проблемой, полностью решенной только в случае двух с половиной степеней свободы [68]. Диффузия в областях, не содержащих резонансов низкого порядка, в случае произвольной размерности установлена в [69].

В заключение упомянем еще одно приложение сепаратрисного отображения. Согласно [29], [48], [51] для (пока специальных случаев) априори неустойчивых систем с двумя с половиной степенями свободы построен большой набор траекторий, проекции которых на ось $y$ при малых $\varepsilon>0$ ведут себя как траектории броуновского движения. Это показывает, что предложенный Чириковым термин "диффузия" достаточно адекватен для обсуждаемого явления.

\section{Список литературы}

[1] В. С. Афраймович, Л.П. Шильников, "О малых периодических возмущениях автономных систем", Докл. АН СССР, 214:4 (1974), 739-742; англ. пер.: V.S. Afrămovich, L. P. Shil'nikov, "On small periodic perturbations of autonomous systems", Soviet Math. Dokl., 15 (1974), 206-211.

[2] В. М. Алексеев, "Квазислучайные динамические системы. І. Квазислучайные диффеоморфизмы", Матем. сб., 76(118):1 (1968), 72-134; "II. Одномерные нелинейные колебания в периодически возмущаемом поле", 77(119):4 (1968), 545-601; "III. Квазислучайные колебания одномерных осцилляторов", 78(120):1 (1969), 3-50; англ. пер.: V. M. Alekseev, "Quasirandom dynamical systems. I. Quasirandom diffeomorphisms", Math. USSR-Sb., 5:1 (1968), 73-128; "II. One-dimensional nonlinear oscillations in a field with periodic perturbation", Math. USSR-Sb., 6:4 (1968), 505-560; "III. Quasirandom oscillations of one-dimensional oscillators", Math. USSR-Sb., 7:1 (1969), 1-43.

[3] В.И. Арнольд, "О неустойчивости динамических систем со многими степенями свободы", Докл. АН ССCP, 156 (1964), 9-12; англ. пер.: V. I. Arnol'd, "Instability of dynamical systems with several degrees of freedom", Soviet Math. Dokl., 5 (1964), $581-585$. 
[4] В.И. Арнольд, Математические методъ классической механики, Наука, М., 1974, 431 с.; англ. пер.: V. I. Arnold, Mathematical methods of classical mechanics, Grad. Texts in Math., 60, Springer-Verlag, New York, 1978, xvi+462 pp.

[5] В. И. Арнольд, В. В. Козлов, А. И. Нейштадт, Динамические системы - 3, Итоги науки и техн. Сер. Соврем. пробл. матем. Фундам. направления, 3, ВИНИТИ, М., 1985, 5-290; англ. пер.: V. I. Arnol'd, V. V. Kozlov, A. I. Neĭshtadt, "Mathematical aspects of classical and celestial mechanics", Dynamical systems, III, Encyclopaedia Math. Sci., 3, Springer-Verlag, Berlin, 1988, 1-291.

[6] S. Aubry, G. Abramovici, "Chaotic trajectories in the standard map. The concept of anti-integrability", Phys. D, 43:2-3 (1990), 199-219.

[7] S. Aubry, R.S. MacKay, C. Baesens, "Equivalence of uniform hyperbolicity for symplectic twist maps and phonon gap for Frenkel-Kontorova models", Phys. D, 56:2-3 (1992), 123-134.

[8] C. Baesens, Y.-C. Chen, R. S. MacKay, "Abrupt bifurcations in chaotic scattering: view from the anti-integrable limit", Nonlinearity, 26:9 (2013), 2703-2730.

[9] M. Berti, P. Bolle, "A functional analysis approach to Arnold diffusion", Ann. Inst. H. Poincaré Anal. Non Linéaire, 19:4 (2002), 395-450.

[10] M. Berti, L. Biasco, P. Bolle, "Drift in phase space: a new variational mechanism with optimal diffusion time", J. Math. Pures Appl. (9), 82:6 (2003), 613-664.

[11] U. Bessi, "An approach to Arnold's diffusion through the calculus of variations", Nonlinear Anal., 26:6 (1996), 1115-1135.

[12] M. Bialy, "Maximizing orbits for higher-dimensional convex billiards", J. Mod. Dyn., 3:1 (2009), 51-59.

[13] Дж. Д. Биркгоф, Динамические системы, Издательский дом "Удмуртский университет", Ижевск, 1999, 408 с.; пер. с англ.: G. D. Birkhoff, Dynamical systems, Amer. Math. Soc. Colloq. Publ., 9, Amer. Math. Soc., New York, 1927, viii+295 pp.

[14] С. В. Болотин, "Либрационные движения натуральных динамических систем", Вестн. Моск. ун-та. Сер. 1 Матем. Мех., 1978, № 6, 72-77; англ. пер.: S. V. Воlotin, "Librations of natural dynamic systems", Mosc. Univ. Mech. Bull., 33:5-6 (1978), 49-53.

[15] S. V. Bolotin, "Symbolic dynamics near minimal hyperbolic invariant tori of Lagrangian systems", Nonlinearity, 14:5 (2001), 1123-1140.

[16] S. V. Bolotin, "Shadowing chains of collision orbits", Discrete Contin. Dyn. Syst., 14:2 (2006), 235-260.

[17] S. Bolotin, "Symbolic dynamics of almost collision orbits and skew products of symplectic maps", Nonlinearity, 19:9 (2006), 2041-2063.

[18] S. Bolotin, R. MacKay, "Multibump orbits near the anti-integrable limit for Lagrangian systems", Nonlinearity, 10:5 (1997), 1015-1029.

[19] S. V. Bolotin, R. S. MacKay, "Periodic and chaotic trajectories of the second species for the n-centre problem", Celestial Mech. Dynam. Astronom., 77:1 (2000), 49-75.

[20] S. Bolotin, R. S. MacKay, "Nonplanar second species periodic and chaotic trajectories for the circular restricted three-body problem", Celestial Mech. Dynam. Astronom., 94:4 (2006), 433-449.

[21] S. V. Bolotin, P. Negrini, "Global regularization for the $n$-center problem on a manifold", Discrete Contin. Dyn. Syst., 8:4 (2002), 873-892.

[22] S. V. Bolotin, P. Negrini, "Variational approach to second species periodic solutions of Poincaré of the 3 body problem", Discrete Contin. Dyn. Syst., 33:3 (2013), 1009-1032.

[23] S. Bolotin, P. Negrini, "Shilnikov lemma for a nondegenerate critical manifold of a Hamiltonian system", Regul. Chaotic Dyn., 18:6 (2013), 774-800.

[24] S. V. Bolotin, P. H. Rabinowitz, "A variational construction of chaotic trajectories for a reversible Hamiltonian system", J. Differential Equations, 148:2 (1998), 364-387. 
[25] S. V. Bolotin, D. V. Treschev, "Remarks on the definition of hyperbolic tori of Hamiltonian systems", Regul. Chaotic Dyn., 5:4 (2000), 401-412.

[26] A. Bounemoura, E. Pennamen, "Instability for a priori unstable Hamiltonian systems: a dynamical approach", Discrete Contin. Dyn. Syst., 32:3 (2012), 753-793.

[27] B. Buffoni, E. Séré, "A global condition for quasi-random behavior in a class of conservative systems", Comm. Pure Appl. Math., 49:3 (1996), 285-305.

[28] Л.А. Бунимович, Я.Г. Синай, Н.И. Чернов, "Статистические свойства двумерных гиперболических биллиардов”, УМН, 46:4(280) (1991), 43-92; англ. пер.: L.A. Bunimovich, Ya. G. Sinai, N.I. Chernov, "Statistical properties of two-dimensional hyperbolic billiards", Russian Math. Surveys, 46:4 (1991), 47-106.

[29] O. Castejón, V. Kaloshin, Random iteration of maps on a cylinder and diffusive behavior, 2015, $75 \mathrm{pp}$.

http://www.math.umd.edu/ ${ }^{\sim}$ vkaloshi/papers/random-iteration.pdf

[30] Y.-C. Chen, "Anti-integrability in scattering billiards", Dyn. Syst., 19:2 (2004), 145-159.

[31] Y.-C. Chen, "On topological entropy of billiard tables with small inner scatterers", Adv. Math., 224:2 (2010), 432-460.

[32] C.-Q. Cheng, J. Yan, "Existence of diffusion orbits in a priori unstable Hamiltonian systems", J. Differential Geom., 67:3 (2004), 457-517.

[33] C.-Q. Cheng, J. Yan, "Arnold diffusion in Hamiltonian systems: a priori unstable case", J. Differential Geom., 82:2 (2009), 229-277.

[34] L. Chierchia, G. Gallavotti, "Drift and diffusion in phase space", Ann. Inst. H. Poincaré Phys. Théor., 60:1 (1994), 1-144.

[35] A. Delshams, R. de la Llave, T. M. Seara, A geometric mechanism for diffusion in Hamiltonian systems overcoming the large gap problem: heuristics and rigorous verification on a model, Mem. Amer. Math. Soc., 179, № 844, Amer. Math. Soc., Providence, RI, 2006, viii+141 pp.

[36] A. Delshams, R. de la Llave, T. M. Seara, "Geometric properties of the scattering map of a normally hyperbolic invariant manifold", Adv. Math., 217:3 (2008), 1096-1153.

[37] A. Delshams, G. Huguet, "Geography of resonances and Arnold diffusion in a priori unstable Hamiltonian systems", Nonlinearity, 22:8 (2009), 1997-2077.

[38] A. Delshams, G. Huguet, "A geometric mechanism of diffusion: rigorous verification in a priori unstable Hamiltonian systems", J. Differential Equations, 250:5 (2011), 2601-2623.

[39] Bo Deng, "The Šil'nikov problem, exponential expansion, strong $\lambda$-lemma, $C^{1}$-linearization, and homoclinic bifurcation", J. Differential Equations, 79:2 (1989), 189-231.

[40] R. L. Devaney, "Homoclinic orbits in Hamiltonian systems", J. Differential Equations, 21:2 (1976), 431-438.

[41] P. Duarte, "Plenty of elliptic islands for the standard family of area preserving maps", Ann. Inst. H. Poincaré Anal. Non Linéaire, 11:4 (1994), 359-409.

[42] R. W. Easton, J. D. Meiss, G. Roberts, "Drift by coupling to an anti-integrable limit", Phys. D, 156:3-4 (2001), 201-218.

[43] E. Fontich, P. Martín, "Arnold diffusion in perturbations of analytic integrable Hamiltonian systems", Discrete Contin. Dyn. Syst., 7:1 (2001), 61-84.

[44] G. Gallavotti, G. Gentile, V. Mastropietro, "Hamilton-Jacobi equation, heteroclinic chains and Arnol'd diffusion in three time scale systems", Nonlinearity, 13:2 (2000), 323-340.

[45] V. Gelfreich, D. Turaev, "Unbounded energy growth in Hamiltonian systems with a slowly varying parameter", Comm. Math. Phys., 283:3 (2008), 769-794.

[46] M. Gidea, C. Robinson, "Diffusion along transition chains of invariant tori and Aubry-Mather sets", Ergodic Theory Dynam. Systems, 33:5 (2013), 1401-1449. 
[47] C. Golé, Symplectic twist maps. Global variational techniques, Adv. Ser. Nonlinear Dynam., 18, World Scientific Publishing Co., Inc., River Edge, NJ, 2001, xviii+305 pp.

[48] M. Guardia, V. Kaloshin, J. Zhang, A second order expansion of the separatrix map for trigonometric perturbations of a priori unstable systems, 2015, 50 pp., arXiv: $1503.08301 \mathrm{v} 2$.

[49] V. Kaloshin, M. Levi, "Geometry of Arnold diffusion", SIAM Rev., 50:4 (2008), 702-720.

[50] V. Kaloshin, Ke Zhang, Dynamics of the dominant Hamiltonian, with applications to Arnold diffusion, 2014 (v2 - 2015), 84 pp., arXiv: 1410.1844.

[51] V. Kaloshin, Jianlu Zhang, Ke Zhang, Normally hyperbolic invariant laminations and diffusive behaviour for the generalized Arnold example away from resonances, 2015, 85 pp., arXiv: 1511.04835.

[52] A. Katok, B. Hasselblatt, Introduction to the modern theory of dynamical systems, Encyclopedia Math. Appl., 54, Cambridge Univ. Press, Cambridge, 1995, xviii+802 pp.

[53] M. Klein, A. Knauf, Classical planar scattering by Coulombic potentials, Lecture Notes in Phys. New Ser. Monogr., M13, Springer-Verlag, Berlin, 1992, v+142 pp.

[54] O. Knill, "Topological entropy of standard type monotone twist maps", Trans. Amer. Math. Soc., 348:8 (1996), 2999-3013.

[55] В. В. Козлов, Д. В. Трещёв, Биллиарды. Генетическое введение в динамику систем с ударами, Изд-во Моск. ун-та, М., 1991, 168 с.; англ. пер.: V. V. Kozlov, D. V. Treshchev, Billiards. A genetic introduction to the dynamics of systems with impacts, Transl. Math. Monogr., 89, Amer. Math. Soc., Providence, RI, 1991, viii+171 pp.

[56] L. M. Lerman, "Complex dynamics and bifurcations in a Hamiltonian system having a transversal homoclinic orbit to a saddle focus", Chaos, 1:2 (1991), 174-180.

[57] R.S. MacKay, J.D. Meiss, "Cantori for symplectic maps near the anti-integrable limit", Nonlinearity, 5:1 (1992), 149-160.

[58] J. N. Mather, "Existence of quasi-periodic orbits for twist homeomorphisms of the annulus", Topology, 21:4 (1982), 457-467.

[59] Д. Макдафф, Д. Саламон, Введение в симплектическую топологию, РХД, Ижевск, 2012, 556 с.; пер. с англ.: D. McDuff, D. Salamon, Introduction to symplectic topology, 2nd ed., Oxford Math. Monogr., The Clarendon Press, Oxford Univ. Press, New York, 1998, x+486 pp.

[60] Z. Nitecki, Differentiable dynamics. An introduction to the orbit structure of diffeomorphisms, The M.I.T. Press, Cambridge, MA-London, 1971, xv+282 pp.

[61] G. N. Piftankin, "Diffusion speed in the Mather problem", Nonlinearity, 19:11 (2006), 2617-2644.

[62] Г.Н. Пифтанкин, Д.В. Трещёв, "Сепаратрисное отображение в гамильтоновых системах", УМН, 62:2(374) (2007), 3-108; англ. пер.: G. N. Piftankin, D. V. Treschev, "Separatrix map in Hamiltonian systems", Russian Math. Surveys, 62:2 (2007), 219-322.

[63] Л.П. Шильников, “Об одной задаче Пуанкаре-Биркгофа”, Матем. сб., 74(116):3 (1967), 378-397; англ. пер.: L. P. Shilnikov, "On a Poincaré-Birkhoff problem", Math. USSR-Sb., 3:3 (1967), 353-371.

[64] L. P. Shilnikov, A. L. Shilnikov, D. V. Turaev, L. O. Chua, Methods of qualitative theory in nonlinear dynamics. Part II, World Sci. Ser. Nonlinear Sci. Ser. A Monogr. Treatises, 5, World Scientific Publishing Co., Inc., River Edge, NJ, 2001, xxiii+565 pp.

[65] S. Smale, "Diffeomorphisms with many periodic points", Differential and combinatorial topology, A symposium in honor of M. Morse, Princeton Univ. Press, Princeton, N.J., 1965, 63-80.

[66] D. Treschev, "Multidimensional symplectic separatrix maps", J. Nonlinear Sci., 12:1 (2002), 27-58. 
[67] D. Treschev, "Trajectories in a neighbourhood of asymptotic surfaces of a priori unstable Hamiltonian systems", Nonlinearity, 15:6 (2002), 2033-2052.

[68] D. Treschev, "Evolution of slow variables in a priori unstable Hamiltonian systems", Nonlinearity, 17:5 (2004), 1803-1841.

[69] D. Treschev, "Arnold diffusion far from strong resonances in multidimensional a priori unstable Hamiltonian systems", Nonlinearity, 25:9 (2012), 2717-2757.

[70] D. Treschev, O. Zubelevich, Introduction to the perturbation theory of Hamiltonian systems, Springer Monogr. Math., Springer-Verlag, Berlin, 2010, x+211 pp.

[71] D. Turaev, "Hyperbolic sets near homoclinic loops to a saddle for systems with a first integral", Regul. Chaotic Dyn., 19:6 (2014), 681-693.

[72] Д. В. Тураев, Л. П. Шильников, "О гамильтоновых системах с гомоклиническими кривыми седла”, Докл. АН СССР, 304:4 (1989), 811-814; англ. пер.: D. V. Turaev, L.P. Shilnikov, "On Hamiltonian systems with homoclinic saddle curves", Soviet Math. Dokl., 39:1 (1989), 165-168.

[73] А.Б. Васильева, В.Ф. Бутузов, Асимптотические разложения решений сингулярно-возмущенных уравнений, Наука, М., 1973, 272 с.

[74] А. П. Веселов, “Интегрируемые отображения”, УМН, 46:5(281) (1991), 3-45; англ. пер.: A. P. Veselov, "Integrable maps", Russian Math. Surveys, 46:5 (1991), 1-51.

[75] P. Walters, An introduction to ergodic theory, Grad. Texts in Math., 79, SpringerVerlag, New York-Berlin, 1982, ix+250 pp.

\section{Сергей Владимирович Болотин}

Поступила в редакцию

(Sergey V. Bolotin)

17.10 .2015

Математический институт им. В. А. Стеклова

Российской академии наук

E-mail: bolotin@mi.ras.ru

\section{Дмитрий Валерьевич Трещёв \\ (Dmitrii V. Treschev)}

Математический институт им. В.А.Стеклова

Российской академии наук

E-mail: treschev@mi.ras.ru 